\title{
Mechanism and Diastereoselectivity of Aziridine Formation from Sulfur Ylides and Imines: A Computational Study
}

\author{
Raphaël Robiette* \\ Unité de Chimie organique et médicinale, Université catholique de Louvain, Place Louis Pasteur 1, \\ B-1348 Louvain-la-Neuve, Belgium. \\ Email:robiette@chim.ucl.ac.be
}

\section{Supporting Information}

\author{
I. Gaussian 03 reference S2 \\ II. MOLPRO reference S2 \\ III. Model reaction results S3 \\ IV. Energies and geometries $S 4$
}




\section{Gaussian 03 reference}

Gaussian 03, Revision B.04, Frisch, M. J.; Trucks, G. W.; Schlegel, H. B.; Scuseria, G. E.; Robb, M. A.; Cheeseman, J. R.; Montgomery, Jr., J. A.; Vreven, T.; Kudin, K. N.; Burant, J. C.; Millam, J. M.; Iyengar, S. S.; Tomasi, J.; Barone, V.; Mennucci, B.; Cossi, M.; Scalmani, G.; Rega, N.; Petersson, G. A.; Nakatsuji, H.; Hada, M.; Ehara, M.; Toyota, K.; Fukuda, R.; Hasegawa, J.; Ishida, M.; Nakajima, T.; Honda, Y.; Kitao, O.; Nakai, H.; Klene, M.; Li, X.; Knox, J. E.; Hratchian, H. P.; Cross, J. B.; Bakken, V.; Adamo, C.; Jaramillo, J.; Gomperts, R.; Stratmann, R. E.; Yazyev, O.; Austin, A. J.; Cammi, R.; Pomelli, C.; Ochterski, J. W.; Ayala, P. Y.; Morokuma, K.; Voth, G. A.; Salvador, P.; Dannenberg, J. J.; Zakrzewski, V. G.; Dapprich, S.; Daniels, A. D.; Strain, M. C.; Farkas, O.; Malick, D. K.; Rabuck, A. D.; Raghavachari, K.; Foresman, J. B.; Ortiz, J. V.; Cui, Q.; Baboul, A. G.; Clifford, S.; Cioslowski, J.; Stefanov, B. B.; Liu, G.; Liashenko, A.; Piskorz, P.; Komaromi, I.; Martin, R. L.; Fox, D. J.; Keith, T.; Al-Laham, M. A.; Peng, C. Y.; Nanayakkara, A.; Challacombe, M.; Gill, P. M. W.; Johnson, B.; Chen, W.; Wong, M. W.; Gonzalez, C.; and Pople, J. A.; Gaussian, Inc., Pittsburgh, 2003.

\section{MOLPRO reference}

MOLPRO, Version 2002.3, Werner H.-J; Knowles, P. J.; Schütz, M.; Lindh, R.; Celani, P.; Korona, T.; Rauhut, G.; Manby, F. R.; Amos, R. D.; Bernhardsson, A.; Berning, A.; Cooper, D. L.; Deegan, M. J. O.; Dobbyn, A. J.; Eckert, F.; Hampel, C.; Hetzer, G.; Lloyd, A. W.; McNicholas, S. J.; Meyer, W.; Mura, M. E.; Nicklaß, A.; Palmieri, P.; Pitzer, R.; Schumann, U.; Stoll, H.; Stone A. J.; Tarroni, R. and Thorsteinsson, T. 


\section{Model reaction results}

Our results for the model reaction $\left(\mathrm{CH}_{2} \mathrm{SMe}_{2}+\mathrm{CH}_{2}=\mathrm{NSO}_{2} \mathrm{H}\right)$ are on the whole very similar to those reported for the corresponding epoxidation reaction $\left(\mathrm{CH}_{2} \mathrm{SMe}_{2}+\mathrm{CH}_{2}=\mathrm{O}\right)$ and are presented in Table SI-1, with the most representative energy profile illustrated in Figure SI-1.

Table SI-1. Relative energies $(\mathrm{kcal} / \mathrm{mol})$ of the stationary points (geometries optimized at the B3LYP/6-31G* $\left(\mathrm{CH}_{3} \mathrm{CN}\right)$ level of theory) for the model aziridination reaction $\mathrm{CH}_{2} \mathrm{SMe}_{2}+$ $\left.\mathrm{CH}_{2}=\mathrm{NSO}_{2} \mathrm{H}\right)$.

\begin{tabular}{|c|c|c|c|c|c|c|}
\hline Level of theory & $\begin{array}{c}\mathrm{CH}_{2} \mathrm{SMe}_{2}+ \\
\mathrm{CH}_{2}=\mathrm{NSO}_{2} \mathrm{H}\end{array}$ & $\begin{array}{l}\text { cisoid } \\
\text { betaine }\end{array}$ & torsional TS & $\begin{array}{l}\text { transoid } \\
\text { betaine }\end{array}$ & $\begin{array}{c}\text { elimination } \\
\text { TS }\end{array}$ & $\begin{array}{c}\mathrm{CH}_{2} \mathrm{CH}_{2}\left(\mathrm{NSO}_{2} \mathrm{H}\right) \\
+\mathrm{Me}_{2} \mathrm{~S}\end{array}$ \\
\hline B3LYP/6-31G* $\left(\mathrm{CH}_{3} \mathrm{CN}\right)$ & 0.0 & -45.8 & -40.2 & -43.3 & -28.0 & -49.2 \\
\hline B3LYP/6-311+G** $\left(\mathrm{CH}_{3} \mathrm{CN}\right)$ & 0.0 & -44.8 & -41.7 & -44.4 & -24.5 & -42.6 \\
\hline B3LYP/6-31G* & 0.0 & -27.4 & -7.3 & -10.2 & -21.6 & -50.7 \\
\hline B3LYP/6-311+G** & 0.0 & -26.5 & -9.6 & -12.1 & -18.6 & -45.9 \\
\hline MP2/6-31G* & 0.0 & -36.1 & -13.6 & -17.0 & -24.5 & -57.8 \\
\hline $\mathrm{MP} 2 / 6-311+\mathrm{G}^{* *}$ & 0.0 & -36.5 & -16.9 & -20.0 & -22.0 & -50.7 \\
\hline MP2/ GTMP2Large & 0.0 & -34.5 & -14.9 & -18.0 & -18.9 & -45.0 \\
\hline QCISD(T)/6-31G* & 0.0 & -33.5 & -11.3 & -14.5 & -24.2 & -55.5 \\
\hline G3(MP2) & 0.0 & -27.1 & -8.8 & -11.6 & -15.5 & -39.6 \\
\hline
\end{tabular}
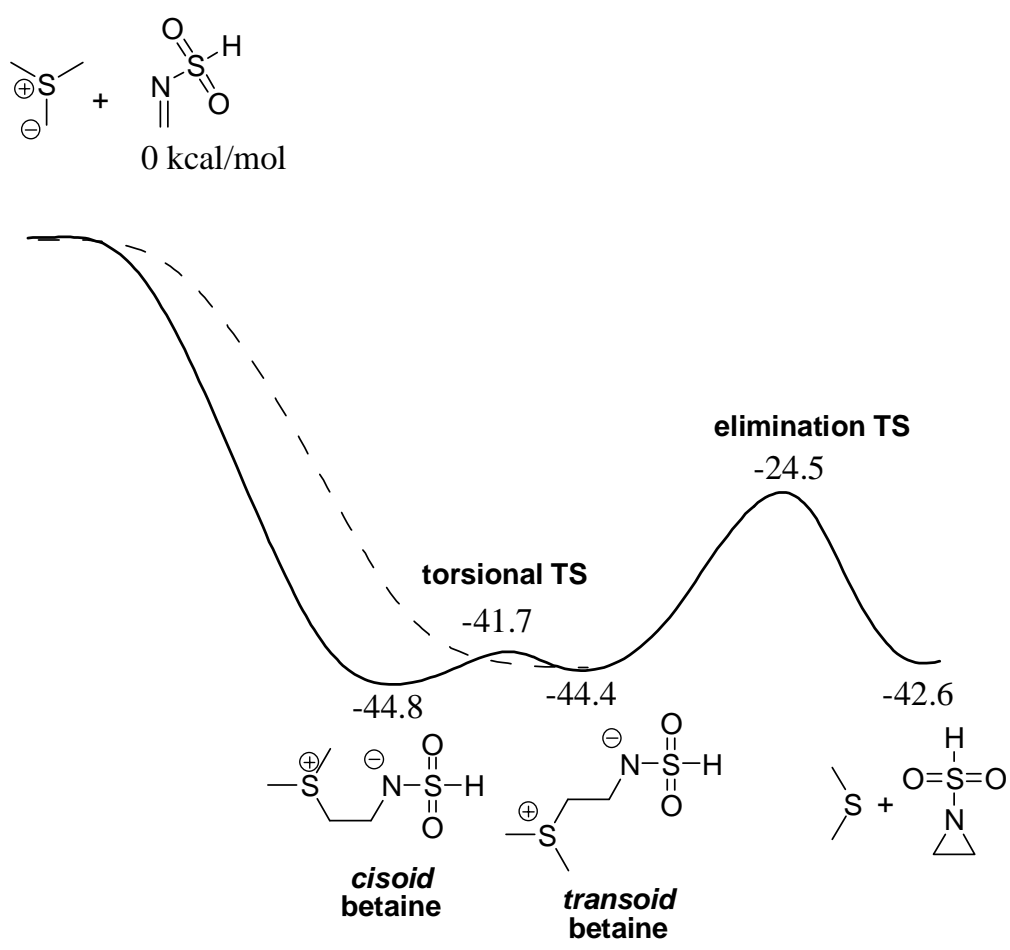

Figure SI-1. Computed potential energy surface for the model aziridination reaction. Energies are obtained at the B3LYP/6-311+G** $\left(\mathrm{CH}_{3} \mathrm{CN}\right) / / \mathrm{B} 3 \mathrm{LYP} / 6-31 \mathrm{G}^{*}\left(\mathrm{CH}_{3} \mathrm{CN}\right)$ level and are given in $\mathrm{kcal} / \mathrm{mol}$ relative to reactants. 


\section{Energies and geometries}

All geometries have been optimized at the B3LYP/6-31G* level of theory including a continuum description of acetonitrile solvent $\left(\mathrm{CH}_{3} \mathrm{CN}\right)$. Energies are given in a.u., geometries as Cartesian coordinates in $\AA$.

\section{A. Model reaction: $\mathrm{Me}_{2} \mathrm{SCH}_{2}+$ $\mathrm{CH}_{2} \mathrm{NSO}_{2} \mathrm{H}$}

\section{$\mathrm{CH}_{2} \mathrm{NSO}_{2} \mathrm{H}$}

$\mathrm{E}\left(\mathrm{B} 3 \mathrm{LYP} 6-31 \mathrm{G}^{*}\right)=-643.18313$ $\mathrm{E}\left(\mathrm{B} 3 \mathrm{LYP} 6-311+\mathrm{G}^{* *}\right)=$ $-643.288792$

$\mathrm{E}\left(\mathrm{B} 3 \mathrm{LYP} 6-31 \mathrm{G} *\left(\mathrm{CH}_{3} \mathrm{CN}\right)\right)=$ $-643.205727$

$\mathrm{E}\left(\mathrm{B} 3 \mathrm{LYP} 6-311+\mathrm{G}^{* *}\left(\mathrm{CH}_{3} \mathrm{CN}\right)\right)=$ $-643.3158677$

$\mathrm{E}\left(\mathrm{MP} 26-31 \mathrm{G}^{*}\right)=-641.9574411$

$\mathrm{E}\left(\mathrm{MP} 26-311+\mathrm{G}^{* *}\right)=-642.1250895$

$\mathrm{E}(\mathrm{QCISD}(\mathrm{T})$ 6-31G*) =

$-642.006923$

$\mathrm{E}(\mathrm{G} 3(\mathrm{MP} 2))=-642.5108785$

$\begin{array}{llll}\mathrm{N} & .47247 & .34471 & -.46816 \\ \mathrm{C} & -.62271 & .47800 & .17659 \\ \mathrm{H} & -.96819 & -.19782 & .96404 \\ \mathrm{H} & -1.25450 & 1.33003 & -.07712 \\ \mathrm{~S} & 1.47835 & -.96637 & .01412 \\ \mathrm{O} & 2.74929 & -.36454 & .43699 \\ \mathrm{O} & .78781 & -1.90009 & .91413 \\ \mathrm{H} & 1.64361 & -1.54894 & -1.19571\end{array}$

\section{$\mathrm{CH}_{2} \mathrm{SMe}_{2}$}

$\mathrm{E}\left(\mathrm{B} 3 \mathrm{LYP} 6-31 \mathrm{G}^{*}\right)=-517.228122$

$\mathrm{E}($ B3LYP 6-311+G**) $=$

$-517.298454$

$\mathrm{E}\left(\mathrm{B} 3 \mathrm{LYP} 6-31 \mathrm{G}^{*}\left(\mathrm{CH}_{3} \mathrm{CN}\right)\right)=$

$-517.2358526$

$\mathrm{E}\left(\mathrm{B} 3 \mathrm{LYP} 6-311+\mathrm{G} * *\left(\mathrm{CH}_{3} \mathrm{CN}\right)\right)=$ $-517.3083117$

$\mathrm{E}\left(\mathrm{MP} 26-31 \mathrm{G}^{*}\right)=-516.1801431$

$\mathrm{E}\left(\mathrm{MP} 26-311+\mathrm{G}^{* *}\right)=$

$-516.3304089$

$\mathrm{E}(\mathrm{QCISD}(\mathrm{T})$ 6-31G*) $=$

$-516.2522367$

$\mathrm{E}(\mathrm{G} 3(\mathrm{MP} 2))=-516.5947234$

$\begin{array}{llll}\text { C } & -.93508 & .37932 & -.01594\end{array}$

H $-1.49000 \quad .86682 \quad .77881$

H $-1.43620 \quad-.15219 \quad-.81459$

$\begin{array}{lllll}\mathrm{S} & .72031 & .32465 & -.05173\end{array}$

C $1.44243 \quad-.32713 \quad 1.52807$

H $1.10721-1.36342 \quad 1.61864$

H $\quad 2.53545 \quad-.28932 \quad 1.47523$
$\begin{array}{cccr}\mathrm{H} & 1.08392 & .25857 & 2.38055 \\ \mathrm{C} & 1.46084 & 2.01757 & .07443 \\ \mathrm{H} & 1.08626 & 2.52892 & .96693 \\ \mathrm{H} & 2.55224 & 1.93846 & .11172 \\ \mathrm{H} & 1.15352 & 2.55981 & -.82331\end{array}$

\section{Cisoid betaine}

$\mathrm{E}\left(\mathrm{B} 3 \mathrm{LYP} 6-31 \mathrm{G}^{*}\right)=-1160.45485$

$\mathrm{E}($ B3LYP 6-311+G**) $=$

$-1160.62951$

$\mathrm{E}\left(\mathrm{B} 3 \mathrm{LYP} 6-31 \mathrm{G}^{*}\left(\mathrm{CH}_{3} \mathrm{CN}\right)\right)=$

$-1160.514571$

$\mathrm{E}\left(\mathrm{B} 3 \mathrm{LYP} 6-311+\mathrm{G}^{* *}\left(\mathrm{CH}_{3} \mathrm{CN}\right)\right)=$ $-1160.695556$

$\mathrm{E}\left(\mathrm{MP} 26-31 \mathrm{G}^{*}\right)=-1158.195131$

$\mathrm{E}\left(\mathrm{MP} 26-311+\mathrm{G}^{* *}\right)=-1158.513674$

$\mathrm{E}(\mathrm{QCISD}(\mathrm{T})$ 6-31G*) $=$

$-1158.31252$

$\mathrm{E}(\mathrm{G} 3(\mathrm{MP} 2))=-1159.148829$

C $-1.40393-1.05823-.23545$

$\mathrm{N}-2.21903 \quad 15460-.25062$

H $-1.65870-1.76064-1.04557$

H $-1.47972-1.63123 \quad 69870$

H $\quad .75659-1.48246-.29486$

$\mathrm{H} \quad .20816 \quad-.23574-1.45788$

$\begin{array}{llll}\text { S }-3.48802 & .16712 & .68498\end{array}$

$\begin{array}{llll}\text { O } & -4.68454 & -.58788 & .19842\end{array}$

$\begin{array}{llll}\text { O } & -3.20410 & -.11949 & 2.12917\end{array}$

$\mathrm{H}-3.80342 \quad 1.47953 \quad .57961$

C $\quad .05543 \quad-.65823 \quad-.45953$

$\begin{array}{llll}\text { S } & .47908 & .72783 & .68352\end{array}$

C $\quad .19614 \quad-.00527 \quad 2.32593$

$\begin{array}{lllll}\mathrm{H} & -.88687 & -.09289 & 2.45361\end{array}$

$\begin{array}{llll}\mathrm{H} & .68996 & -.97786 & 2.39853\end{array}$

$\begin{array}{llll}\mathrm{H} & .60175 & .69362 & 3.06191\end{array}$

$\begin{array}{llll}\text { C } & 2.29802 & .74047 & .58257\end{array}$

$\begin{array}{llll}\text { H } & 2.70949 & -.24868 & .79783\end{array}$

$\begin{array}{llll}\text { H } & 2.56357 & 1.06331 & -.42773\end{array}$

H $2.65417 \quad 1.478821 .30571$

\section{Transoid betaine}

$\mathrm{E}\left(\mathrm{B} 3 \mathrm{LYP} 6-31 \mathrm{G}^{*}\right)=-1160.42749$

$\mathrm{E}($ B3LYP 6-311+G**) $=$

$-1160.60654$

$\mathrm{E}\left(\mathrm{B} 3 \mathrm{LYP} 6-31 \mathrm{G}^{*}\left(\mathrm{CH}_{3} \mathrm{CN}\right)\right)=$

$-1160.51052$

$\mathrm{E}\left(\mathrm{B} 3 \mathrm{LYP} 6-311+\mathrm{G}^{* *}\left(\mathrm{CH}_{3} \mathrm{CN}\right)\right)=$

$-1160.695016$

$\mathrm{E}\left(\mathrm{MP} 26-31 \mathrm{G}^{*}\right)=-1158.164699$

$\mathrm{E}\left(\mathrm{MP} 26-311+\mathrm{G}^{* *}\right)=-1158.487366$

\begin{tabular}{|c|c|c|}
\hline \multicolumn{3}{|c|}{$\begin{array}{l}\mathrm{E}\left(\mathrm{QCISD}(\mathrm{T}) 6-31 \mathrm{G}^{*}\right)= \\
-1158.28226\end{array}$} \\
\hline C -1.78671 & -1.66940 & .23066 \\
\hline $\mathrm{N}-2.40082$ & -.34525 & .29939 \\
\hline $\mathrm{H}-2.08730$ & -2.23030 & -.66840 \\
\hline H -2.01906 & -2.30694 & 1.09860 \\
\hline H $\quad-.00021$ & -.78435 & -.66961 \\
\hline H .03433 & -.87999 & 1.11358 \\
\hline$S-3.97478$ & -.29156 & .28174 \\
\hline O -4.62926 & -.82534 & -.95474 \\
\hline O -4.66174 & -.77820 & 1.52038 \\
\hline H -4.11724 & 1.05519 & .26278 \\
\hline C $\quad-.27952$ & -1.39051 & .19760 \\
\hline .73058 & -2.92675 & .15403 \\
\hline C .49330 & -3.52354 & -1.55070 \\
\hline H $\quad-.52836$ & -3.90308 & -1.62625 \\
\hline H .66361 & -2.70956 & -2.26018 \\
\hline H 1.20289 & -4.33927 & -1.71069 \\
\hline C 2.41579 & -2.23615 & .08487 \\
\hline H 2.49552 & -1.52247 & -.73911 \\
\hline H 2.60202 & -1.74726 & 1.04454 \\
\hline H 3.10800 & -3.07115 & -.04824 \\
\hline
\end{tabular}

\section{Torsional TS}

\begin{tabular}{|c|c|c|c|}
\hline & 33LYP 6-3 & $\left.31 G^{*}\right)=-11$ & 160.4229 \\
\hline & 33LYP 6-3 & $\left.311+\mathrm{G}^{* *}\right)=$ & \\
\hline & 60.60252 & & \\
\hline & $\begin{array}{l}\text { 33LYP 6-3 } \\
60.50570\end{array}$ & ${ }_{8}^{31 G} *\left(\mathrm{CH}_{3} \mathrm{C}\right.$ & $\mathrm{CN}))=$ \\
\hline & $\begin{array}{l}\text { 33LYP 6-3 } \\
60.690672\end{array}$ & $311+\mathrm{G}^{*} *(\mathrm{C}$ & $\left.\left.\mathrm{CH}_{3} \mathrm{CN}\right)\right)=$ \\
\hline & MP2 6-31C & $\left.\mathrm{G}^{*}\right)=-1158$ & 8.159324 \\
\hline & MP2 6-311 & $\left.+\mathrm{G}^{* *}\right)=-1$ & 1158.48236 \\
\hline & QCISD(T) & $\left.6-31 \mathrm{G}^{*}\right)=$ & \\
\hline & 58.277225 & & \\
\hline & $\mathrm{G} 3(\mathrm{MP} 2))$ & $=-1159.11$ & 965 \\
\hline $\mathrm{C}$ & -1.82822 & -1.60653 & .42528 \\
\hline $\mathrm{N}$ & -2.42739 & -.28532 & .24759 \\
\hline $\mathrm{H}$ & -2.16448 & -2.33897 & -.32248 \\
\hline $\mathrm{H}$ & -2.06872 & -2.04879 & 1.40829 \\
\hline $\mathrm{H}$ & .12128 & -1.96060 & -.58743 \\
\hline $\mathrm{H}$ & -.05270 & -.37279 & .20311 \\
\hline S & -4.00133 & -.22742 & .19292 \\
\hline $\mathrm{O}$ & -4.63852 & -.93475 & -.96324 \\
\hline $\mathrm{O}$ & -4.71185 & -.52942 & 1.47680 \\
\hline $\mathrm{H}$ & -4.13366 & 1.10270 & -.02615 \\
\hline $\mathrm{C}$ & -.29865 & -1.43577 & .27547 \\
\hline S & .60642 & -2.00828 & 1.77092 \\
\hline $\mathrm{C}$ & .73511 & -3.80695 & 1.51409 \\
\hline
\end{tabular}




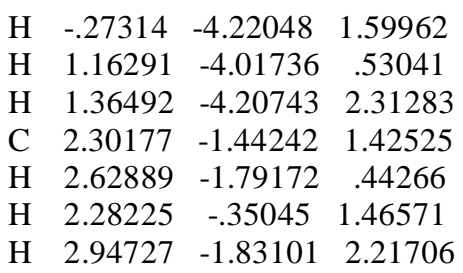

\section{Elimination TS}

E(B3LYP 6-31G*) $=-1160.44575$ $\mathrm{E}\left(\right.$ B3LYP $\left.6-311+\mathrm{G}^{* *}\right)=$

$-1160.61694$

$\mathrm{E}\left(\mathrm{B} 3 \mathrm{LYP} 6-31 \mathrm{G} *\left(\mathrm{CH}_{3} \mathrm{CN}\right)\right)=$ $-1160.486197$

$\mathrm{E}\left(\mathrm{B} 3 \mathrm{LYP} 6-311+\mathrm{G} * *\left(\mathrm{CH}_{3} \mathrm{CN}\right)\right)=$ $-1160.663273$

$\mathrm{E}\left(\mathrm{MP} 26-31 \mathrm{G}^{*}\right)=-1158.176619$

$\mathrm{E}\left(\mathrm{MP} 26-311+\mathrm{G}^{* *}\right)=-1158.490488$

$\mathrm{E}(\mathrm{QCISD}(\mathrm{T}) 6-31 \mathrm{G} *)=$

$-1158.297777$

$\mathrm{E}(\mathrm{G} 3(\mathrm{MP} 2))=-1159.130315$

$\begin{array}{cccc}\mathrm{C} & -1.23805 & -1.68237 & 1.00477 \\ \mathrm{~N} & -1.52562 & -.24053 & .92253 \\ \mathrm{H} & -2.07897 & -2.36613 & 1.01546 \\ \mathrm{H} & -.55380 & -1.90282 & 1.83424 \\ \mathrm{H} & -1.25160 & -1.46842 & -1.18374 \\ \mathrm{H} & .31009 & -.90754 & -.37903 \\ \mathrm{~S} & -3.03833 & .16835 & .49949 \\ \mathrm{O} & -3.65307 & -.72094 & -.51865 \\ \mathrm{O} & -3.88892 & .42606 & 1.68725 \\ \mathrm{H} & -2.79830 & 1.37069 & -.07136 \\ \mathrm{C} & -.60324 & -1.48626 & -.30200 \\ \mathrm{~S} & .57030 & -3.43457 & -1.03036 \\ \mathrm{C} & 1.16116 & -2.74366 & -2.61788 \\ \mathrm{H} & .31965 & -2.73590 & -3.30889 \\ \mathrm{H} & 1.52731 & -1.72048 & -2.47073 \\ \mathrm{H} & 1.96075 & -3.37478 & -3.01653 \\ \mathrm{C} & 2.06021 & -3.27293 & .01024 \\ \mathrm{H} & 2.38853 & -2.23104 & .04296 \\ \mathrm{H} & 1.79024 & -3.60537 & 1.01942 \\ \mathrm{H} & 2.85523 & -3.91030 & -.37904\end{array}$

\section{Aziridine}

$\mathrm{E}(\mathrm{B} 3 \mathrm{LYP}$ 6-31G*) $=-682.478537$

$\mathrm{E}\left(\right.$ B3LYP $\left.6-311+\mathrm{G}^{* *}\right)=$

$-682.593884$

$\mathrm{E}\left(\mathrm{B} 3 \mathrm{LYP} 6-31 \mathrm{G} *\left(\mathrm{CH}_{3} \mathrm{CN}\right)\right)=$ $-682.5006733$

$\mathrm{E}\left(\mathrm{B} 3 \mathrm{LYP} 6-311+\mathrm{G} * *\left(\mathrm{CH}_{3} \mathrm{CN}\right)\right)=$ $-682.6199566$

$\mathrm{E}\left(\mathrm{MP} 26-31 \mathrm{G}^{*}\right)=-681.1094863$

$\mathrm{E}\left(\mathrm{MP} 26-311+\mathrm{G}^{* *}\right)=-681.3072517$

$\mathrm{E}\left(\mathrm{QCISD}(\mathrm{T}) 6-31 \mathrm{G}^{*}\right)=$

$-681.1709707$

$\mathrm{E}(\mathrm{G} 3(\mathrm{MP} 2))=-681.760112$

$\begin{array}{llll}\text { C } & -1.36040 & -1.12854 & .00883\end{array}$

$\begin{array}{llll}\mathrm{C} & .07544 & -.79996 & -.14980\end{array}$

$\begin{array}{llll}\mathrm{N} & -.93238 & .29519 & -.06917\end{array}$
$\begin{array}{cccc}\mathrm{H} & -1.95732 & -1.43890 & -.84424 \\ \mathrm{H} & -1.68257 & -1.49703 & .97941 \\ \mathrm{H} & .72504 & -.95921 & .70794 \\ \mathrm{H} & .55829 & -.86333 & -1.12146 \\ \mathrm{~S} & -.94692 & 1.20257 & 1.35939 \\ \mathrm{O} & -.88704 & .35642 & 2.56227 \\ \mathrm{O} & .05222 & 2.26618 & 1.19825 \\ \mathrm{H} & -2.18735 & 1.73365 & 1.25281\end{array}$

$\mathrm{SMe}_{2}$

$\mathrm{E}\left(\mathrm{B} 3 \mathrm{LYP} 6-31 \mathrm{G}^{*}\right)=-478.013547$

$\mathrm{E}\left(\right.$ B3LYP $\left.6-311+\mathrm{G}^{* *}\right)=$

$-478.066546$

$\mathrm{E}\left(\mathrm{B} 3 \mathrm{LYP} 6-31 \mathrm{G} *\left(\mathrm{CH}_{3} \mathrm{CN}\right)\right)=$

$-478.0193283$

$\mathrm{E}\left(\mathrm{B} 3 \mathrm{LYP} 6-311+\mathrm{G}^{* *}\left(\mathrm{CH}_{3} \mathrm{CN}\right)\right)=$ $-478.0720787$

$\mathrm{E}\left(\mathrm{MP} 26-31 \mathrm{G}^{*}\right)=-477.1201241$

$\mathrm{E}\left(\mathrm{MP} 26-311+\mathrm{G}^{* *}\right)=-477.2291121$

$\mathrm{E}\left(\mathrm{QCISD}(\mathrm{T}) 6-31 \mathrm{G}^{*}\right)=$

$-477.1765785$

$\mathrm{E}(\mathrm{G} 3(\mathrm{MP} 2))=-477.4086448$

$\begin{array}{lllll}\mathrm{S} & .00000 & .00000 & -.57639\end{array}$

$\begin{array}{llll}\text { C } & .00000 & 1.42505 & .57072\end{array}$

$\begin{array}{llll}\mathrm{H} & .00000 & 2.32780 & -.04836\end{array}$

H $-.89623 \quad 1.42722 \quad 1.19780$

$\begin{array}{llll}\mathrm{H} & .89623 & 1.42722 & 1.19780\end{array}$

C $.00000 \quad-1.42505 \quad .57072$

$\mathrm{H} \quad .00000 \quad-2.32780-.04836$

H $\quad .89623 \quad-1.42722 \quad 1.19780$

H $-.89623 \quad-1.42722 \quad 1.19780$

\section{B. Semi-stabilized ylide reaction: $\mathrm{PhCHSMe}_{2}+$ $\mathrm{PhCH}=\mathrm{NSO}_{2} \mathrm{Me}$}

\section{$\mathrm{PhCHSMe}_{2}$}

$\mathrm{E}\left(\mathrm{B} 3 \mathrm{LYP} 6-31 \mathrm{G}^{*}\right)=-748.241185$

$\mathrm{E}($ B3LYP 6-311+G**) $=$

$-748.415080$

$\mathrm{E}\left(\mathrm{B} 3 \mathrm{LYP} 6-31 \mathrm{G} *\left(\mathrm{CH}_{3} \mathrm{CN}\right)\right)=$

$-748.305852$

$\mathrm{E}\left(\right.$ B3LYP 6-311+G** $\left.\left(\mathrm{CH}_{3} \mathrm{CN}\right)\right)=$

$-748.428871$

$\mathrm{E}(\mathrm{B} 3 \mathrm{LYP} 6-311+\mathrm{G} * *(\mathrm{THF}))=$ $-748.426629$

$\begin{array}{llll}\text { C } & 1.30506 & -.06226 & 0.00000\end{array}$

$\begin{array}{llll}\mathrm{H} & 2.24433 & -.60509 & 0.00000\end{array}$

$\begin{array}{llll}\mathrm{S} & 1.57531 & 1.59907 & 0.00000\end{array}$

$\begin{array}{llll}\text { C } & .72467 & 2.47149 & 1.39616\end{array}$

$\begin{array}{llll}\mathrm{H} & 1.15161 & 2.06681 & 2.31694\end{array}$

$\mathrm{H} \quad .95173 \quad 3.53915 \quad 1.31552$

$\begin{array}{llll}\mathrm{H} & -.35453 & 2.30879 & 1.37498\end{array}$ $\begin{array}{llll}\text { C } & .72467 & 2.47149 & -1.39616\end{array}$

$\begin{array}{llll}\mathrm{H} & -.35453 & 2.30879 & -1.37498\end{array}$

H $\quad .95173 \quad 3.53915-1.31552$

H $1.15161 \quad 2.06681 \quad-2.31694$

$\begin{array}{llll}\text { C } & -2.30770 & -2.39849 & 0.00000\end{array}$

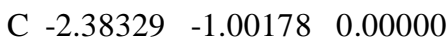

$\begin{array}{llll}\text { C } & -1.23065 & -.21637 & 0.00000\end{array}$

$\begin{array}{llll}\text { C } & .06456 & -.79629 & 0.00000\end{array}$

$\begin{array}{llll}\text { C } & .11524 & -2.21873 & 0.00000\end{array}$

$\begin{array}{llll}\text { C } & -1.03935 & -2.99528 & 0.00000\end{array}$

$\begin{array}{llll}\mathrm{H} & -3.20935 & -3.00602 & 0.00000\end{array}$

$\begin{array}{llll}\text { H } & -3.35465 & -.50997 & 0.00000\end{array}$

$\begin{array}{llll}\mathrm{H} & -1.34553 & .86375 & 0.00000\end{array}$

$\begin{array}{llll}\mathrm{H} & 1.08851 & -2.70786 & 0.00000\end{array}$

$\begin{array}{llll}\mathrm{H} & -.94990 & -4.08059 & 0.00000\end{array}$

\section{$\mathrm{PhCHSMe}_{2}$ (conformer)}

$\mathrm{E}\left(\mathrm{B} 3 \mathrm{LYP} 6-31 \mathrm{G}^{*}\right)=-748.286050$

$\mathrm{E}\left(\right.$ B3LYP $\left.6-311+\mathrm{G}^{* *}\right)=$

$-748.41383384$

$\mathrm{E}\left(\mathrm{B} 3 \mathrm{LYP} 6-31 \mathrm{G} *\left(\mathrm{CH}_{3} \mathrm{CN}\right)\right)=$

$-748.305732$

$\mathrm{E}\left(\mathrm{B} 3 \mathrm{LYP} 6-311+\mathrm{G}^{* *}\left(\mathrm{CH}_{3} \mathrm{CN}\right)\right)=$

$-748.430070$

$\begin{array}{lccc}\mathrm{C} & 1.38874 & -.09277 & -.02775 \\ \mathrm{H} & 2.32107 & -.65033 & -.06000 \\ \mathrm{~S} & 1.49403 & 1.58898 & -.02009 \\ \mathrm{C} & 2.49979 & 2.15641 & -1.46295 \\ \mathrm{H} & 1.91408 & 1.93364 & -2.35773 \\ \mathrm{H} & 2.66984 & 3.23480 & -1.38138 \\ \mathrm{H} & 3.45099 & 1.61630 & -1.48786 \\ \mathrm{C} & 2.66450 & 2.11277 & 1.30502 \\ \mathrm{H} & 3.60713 & 1.56617 & 1.20401 \\ \mathrm{H} & 2.83463 & 3.19125 & 1.22721 \\ \mathrm{H} & 2.18846 & 1.87467 & 2.25897 \\ \mathrm{C} & -2.27700 & -2.35839 & .03072 \\ \mathrm{C} & -2.32238 & -.95808 & .03400 \\ \mathrm{C} & -1.15620 & -.19696 & .01295 \\ \mathrm{C} & .13060 & -.80054 & -.00987 \\ \mathrm{C} & .15032 & -2.22305 & -.01435 \\ \mathrm{C} & -1.02107 & -2.97682 & .00545 \\ \mathrm{H} & -3.19035 & -2.94801 & .04659 \\ \mathrm{H} & -3.28418 & -.44705 & .05232 \\ \mathrm{H} & -1.23700 & .88739 & .01387 \\ \mathrm{H} & 1.11250 & -2.73303 & -.03292 \\ \mathrm{H} & -.95078 & -4.06370 & .00129\end{array}$

\section{$\mathrm{PhCH}=\mathrm{NSO}_{2} \mathrm{Me}$}

$\mathrm{E}(\mathrm{B} 3 \mathrm{LYP}$ 6-31G*) $=-913.525259$

$\mathrm{E}\left(\right.$ B3LYP $\left.6-311+\mathrm{G}^{* *}\right)=$

$-913.755440$

$\mathrm{E}\left(\mathrm{B} 3 \mathrm{LYP} 6-31 \mathrm{G} *\left(\mathrm{CH}_{3} \mathrm{CN}\right)\right)=$

$-913.612311$

$\mathrm{E}\left(\mathrm{B} 3 \mathrm{LYP} 6-311+\mathrm{G}^{* *}\left(\mathrm{CH}_{3} \mathrm{CN}\right)\right)=$ $-913.783056$

$\mathrm{E}(\mathrm{B} 3 \mathrm{LYP} 6-311+\mathrm{G} * *(\mathrm{THF}))=$ $-913.778304$

$\begin{array}{llll}\mathrm{N} & .49731 & .36124 & -.24777\end{array}$ 


$\begin{array}{lccc}\mathrm{C} & -.70932 & .52257 & .18799 \\ \mathrm{H} & -1.27386 & -.30607 & .62845 \\ \mathrm{~S} & 1.18859 & -1.17449 & -.02743 \\ \mathrm{O} & 2.32400 & -.99136 & .89789 \\ \mathrm{O} & .18549 & -2.20087 & .32363 \\ \mathrm{C} & -2.77451 & 4.23543 & -.02508 \\ \mathrm{C} & -3.39880 & 3.11040 & .52263 \\ \mathrm{C} & -2.70991 & 1.89968 & .59196 \\ \mathrm{C} & -1.39068 & 1.80991 & .11189 \\ \mathrm{C} & -.76612 & 2.94998 & -.43214 \\ \mathrm{C} & -1.45744 & 4.15412 & -.50047 \\ \mathrm{H} & -3.31254 & 5.17845 & -.08330 \\ \mathrm{H} & -4.41842 & 3.17774 & .89214 \\ \mathrm{H} & -3.18788 & 1.01937 & 1.01627 \\ \mathrm{H} & .25407 & 2.87559 & -.79616 \\ \mathrm{H} & -.97579 & 5.03196 & -.92325 \\ \mathrm{C} & 1.83350 & -1.51531 & -1.66129 \\ \mathrm{H} & 2.39622 & -2.44987 & -1.58077 \\ \mathrm{H} & 1.00064 & -1.62656 & -2.35923 \\ \mathrm{H} & 2.49046 & -.69387 & -1.95474\end{array}$

$\mathrm{PhCH}=\mathrm{NSO}_{2} \mathrm{Me}$ (conformer)

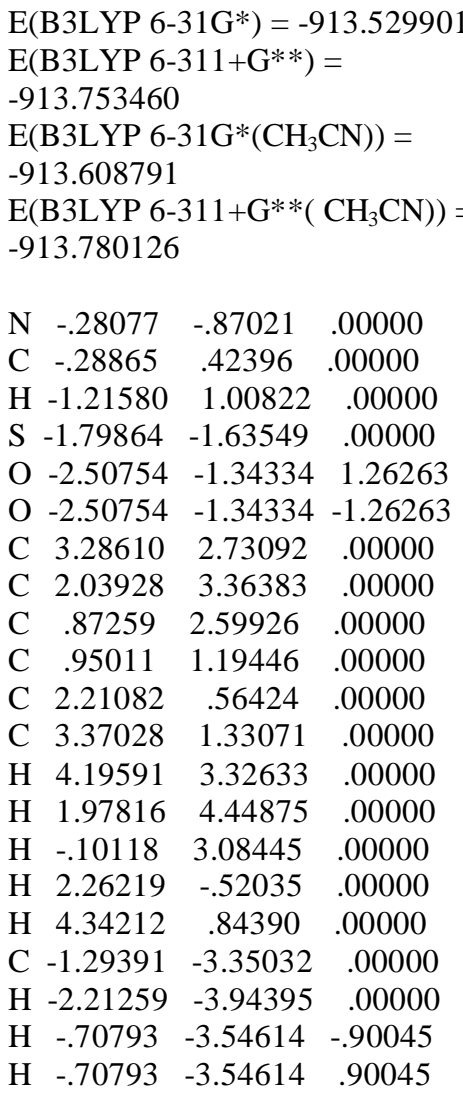

\section{Syn transoid addition TS}

$\mathrm{E}\left(\mathrm{B} 3 \mathrm{LYP} 6-31 \mathrm{G}^{*}\right)=-1661.871676$

$\mathrm{E}\left(\mathrm{B} 3 \mathrm{LYP} 6-311+\mathrm{G}^{* *}\right)=$

$-1662.163485$

$\mathrm{E}\left(\mathrm{B} 3 \mathrm{LYP} 6-31 \mathrm{G}^{*}\left(\mathrm{CH}_{3} \mathrm{CN}\right)\right)=$

$-1661.909103$

$\mathrm{E}\left(\mathrm{B} 3 \mathrm{LYP} 6-311+\mathrm{G}^{* *}\left(\mathrm{CH}_{3} \mathrm{CN}\right)\right)=$

$-1662.202080$

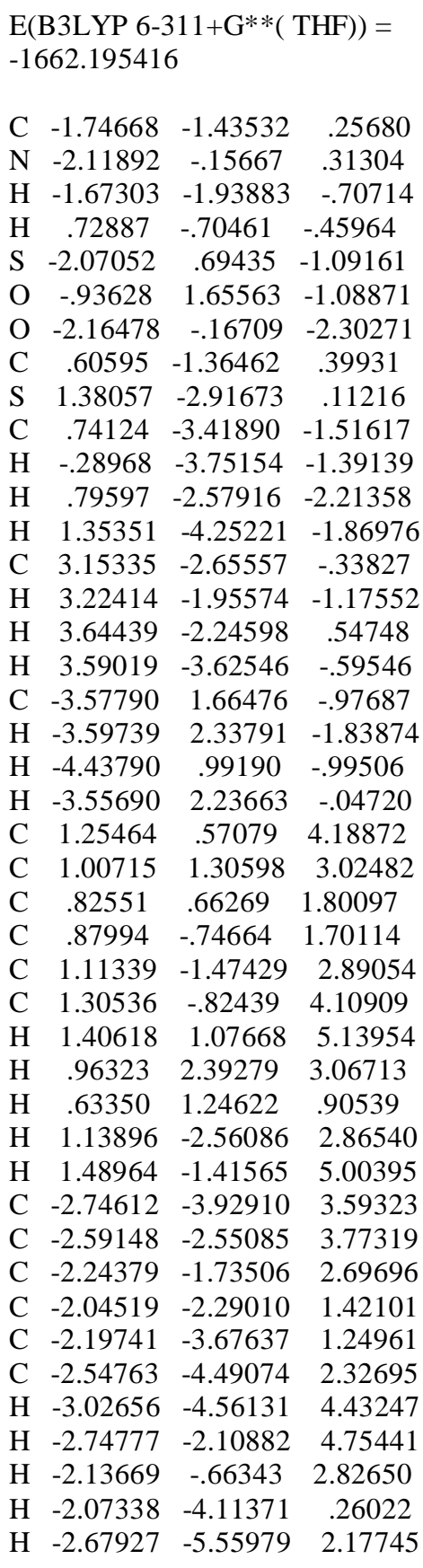

\section{Syn cisoid addition TS}

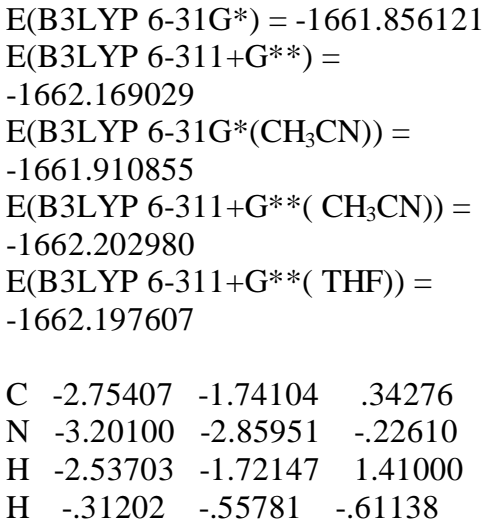

$\begin{array}{llll}S & -3.11085 & -4.25071 & .65964\end{array}$

$\begin{array}{lllll}\mathrm{O} & -2.34229 & -5.25410 & -.11749\end{array}$

$\begin{array}{llll}\mathrm{O} & -2.67473 & -4.03631 & 2.06371\end{array}$

$\begin{array}{llll}\text { C } & -.39834 & -1.31789 & .16538\end{array}$

$\begin{array}{lllll}\mathrm{S} & .33435 & -2.82997 & -.34902\end{array}$

C $\quad-.35577 \quad-3.05562 \quad-2.01474$

H $\quad-1.42149 \quad-3.24904 \quad-1.89204$

H $\quad-.18060 \quad-2.15470 \quad-2.60869$

$\begin{array}{llll}\mathrm{H} & .14018 & -3.91801 & -2.46607\end{array}$

$\begin{array}{llll}\text { C } & 2.10612 & -2.54820 & -.78244\end{array}$

H $2.18688 \quad-1.72353 \quad-1.49594$

H $2.62688-2.30566 \quad .14680$

H $\quad 2.50666 \quad-3.47558 \quad-1.20432$

$\begin{array}{llll}\text { C } & -4.82365 & -4.80042 & .69472\end{array}$

$\begin{array}{llll}\mathrm{H} & -4.84007 & -5.78284 & 1.17561\end{array}$

$\mathrm{H} \quad-5.41210 \quad-4.08298 \quad 1.27086$

H $\quad-5.19587 \quad-4.86955 \quad-.32929$

$\begin{array}{llll}\text { C } & .44552 & .09590 & 4.14321\end{array}$

$\begin{array}{llll}\text { C } & .36727 & .97995 & 3.06239\end{array}$

$\begin{array}{llll}\text { C } & .12960 & .50397 & 1.77278\end{array}$

$\begin{array}{llll}\text { C } & -.05805 & -.87376 & 1.52136\end{array}$

$\begin{array}{llll}\text { C } & 00406 & -1.75082 & 2.63021\end{array}$

$\begin{array}{llll}\text { C } & .26393 & -1.27305 & 3.91352\end{array}$

H $\quad .64249 \quad .46630 \quad 5.14650$

$\begin{array}{llll}\mathrm{H} & .50514 & 2.04779 & 3.22078\end{array}$

$\begin{array}{llll}\mathrm{H} & .09058 & 1.20144 & .93908\end{array}$

$\begin{array}{llll}\mathrm{H} & -.17783 & -2.81199 & 2.48440\end{array}$

H $\quad .31239 \quad-1.97555 \quad 4.74344$

$\begin{array}{llll}\text { C } & -3.97015 & 2.00722 & -1.34502\end{array}$

$\begin{array}{llll}\text { C } & -3.95958 & .85592 & -2.14268\end{array}$

$\begin{array}{llll}\text { C } & -3.54536 & -.36342 & -1.61035\end{array}$

$\begin{array}{llll}\text { C } & -3.12786 & -.45089 & -.27081\end{array}$

$\begin{array}{llll}\text { C } & -3.14208 & .70920 & .52287\end{array}$

$\begin{array}{llll}\text { C } & -3.56044 & 1.92925 & -.01101\end{array}$

H $\quad-4.30270 \quad 2.95577 \quad-1.75998$

$\begin{array}{llll}\mathrm{H} & -4.28399 & .90907 & -3.17947\end{array}$

$\mathrm{H} \quad-3.55440 \quad-1.26041 \quad-2.22188$

$\begin{array}{llll}\mathrm{H} & -2.83843 & .64789 & 1.56429\end{array}$

$\begin{array}{llll}\mathrm{H} & -3.57612 & 2.81623 & .61777\end{array}$

\section{Syn cisoid addition TS (conformer)}

$\mathrm{E}\left(\mathrm{B} 3 \mathrm{LYP} 6-31 \mathrm{G}^{*}\right)=$

$\mathrm{E}\left(\mathrm{B} 3 \mathrm{LYP} 6-311+\mathrm{G}^{* *}\right)=$

$-1662.162426$

$\mathrm{E}\left(\mathrm{B} 3 \mathrm{LYP} 6-31 \mathrm{G} *\left(\mathrm{CH}_{3} \mathrm{CN}\right)\right)=$

$-1661.905722$

$\mathrm{E}\left(\mathrm{B} 3 \mathrm{LYP} 6-311+\mathrm{G}^{* *}\left(\mathrm{CH}_{3} \mathrm{CN}\right)\right)=$ 1662.195898

$\begin{array}{lcll}\mathrm{C} & -1.88289 & -1.77027 & .25909 \\ \mathrm{~N} & -2.28776 & -2.89947 & .83386 \\ \mathrm{H} & -1.95624 & -.82445 & .79487 \\ \mathrm{H} & .45583 & -.75157 & -.51107 \\ \mathrm{~S} & -2.72113 & -2.82448 & 2.41919 \\ \mathrm{O} & -1.88939 & -3.79066 & 3.18401 \\ \mathrm{O} & -2.78939 & -1.44213 & 2.95302 \\ \mathrm{C} & .41402 & -1.35566 & .39424 \\ \mathrm{~S} & 1.22890 & -2.85019 & -.03816 \\ \mathrm{C} & 3.05213 & -2.69511 & .19023 \\ \mathrm{H} & 3.42029 & -2.00888 & -.57587 \\ \mathrm{H} & 3.26698 & -2.31216 & 1.19101\end{array}$ 


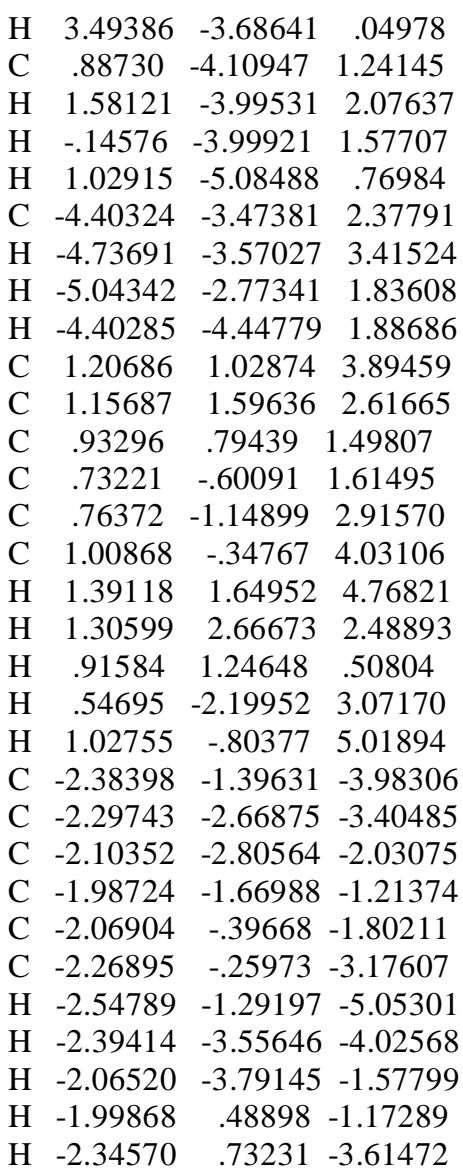

\section{Anti cisoid addition TS}

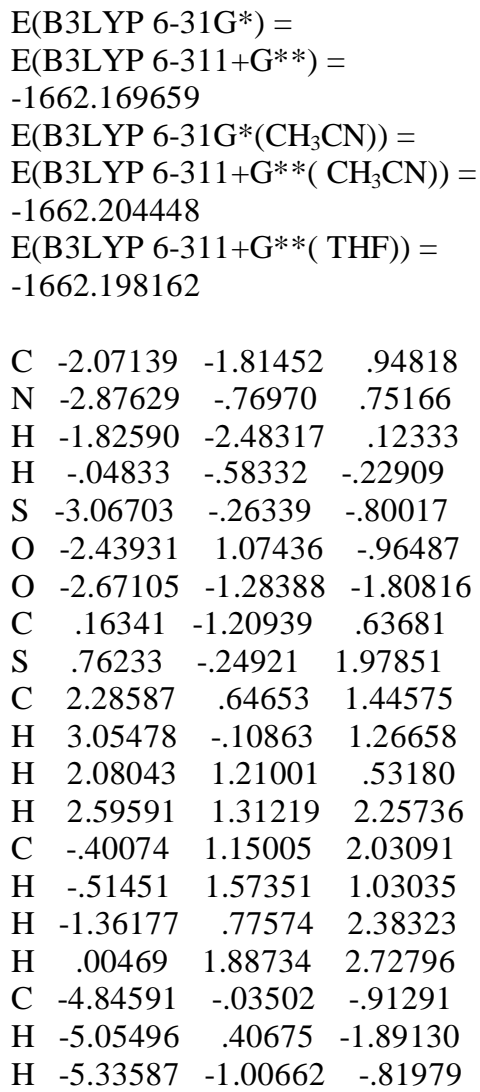

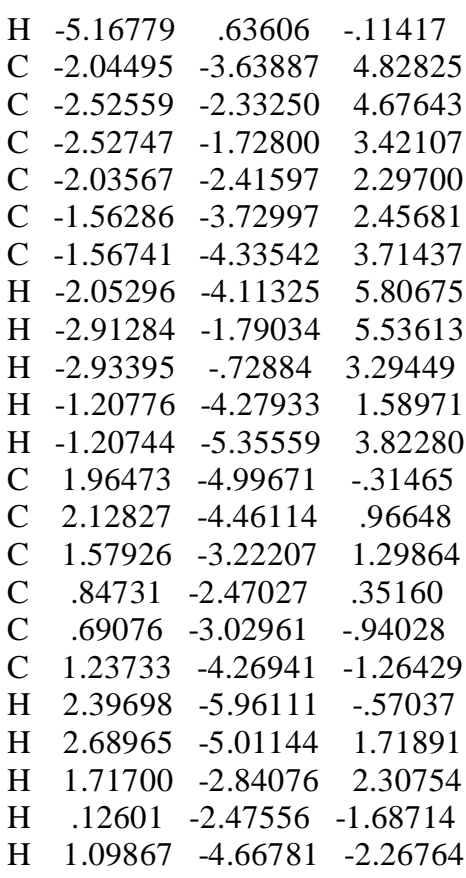

\section{Anti cisoid addition TS (conformer)}

$\mathrm{E}\left(\mathrm{B} 3 \mathrm{LYP} 6-31 \mathrm{G}^{*}\right)=-1661.871955$
$\mathrm{E}\left(\mathrm{B} 3 \mathrm{LYP} 6-311+\mathrm{G}^{* *}\right)=$
-1662.164661
$\mathrm{E}\left(\mathrm{B} 3 \mathrm{LYP} 6-31 \mathrm{G}^{*}\left(\mathrm{CH}_{3} \mathrm{CN}\right)\right)=$
-1661.910596
$\mathrm{E}\left(\mathrm{B} 3 \mathrm{LYP} 6-311+\mathrm{G}^{* *}\left(\mathrm{CH}_{3} \mathrm{CN}\right)\right)=$
-1662.202266

$\begin{array}{llll}\text { C } & -2.85447 & -1.88547 & .69334\end{array}$

$\begin{array}{llll}\mathrm{N} & -3.37348 & -.95744 & 1.48694\end{array}$

$\mathrm{H} \quad-2.92326 \quad-1.81993 \quad-.39041$

$\begin{array}{llll}\mathrm{H} & -.10515 & -2.47703 & .25632\end{array}$

$\begin{array}{llll}\text { S } & -3.92695 & .41635 & .76176\end{array}$

$\begin{array}{llll}\mathrm{O} & -2.99322 & .93813 & -.27445\end{array}$

$\begin{array}{llll}\mathrm{O} & -5.33138 & .23646 & .30979\end{array}$

$\begin{array}{llll}\text { C } & -.44334 & -1.45805 & .43765\end{array}$

$\begin{array}{llll}\mathrm{S} & -.10662 & -.39952 & -.91527\end{array}$

C $\quad 1.57521 \quad-.80302 \quad-1.54664$

H $\quad 2.28226 \quad-.51772 \quad-.76414$

H $\quad 1.64850 \quad-1.87377 \quad-1.75714$

H $\quad 1.75764 \quad-.21258 \quad-2.44996$

C $\quad-1.09077 \quad-1.02799 \quad-2.31548$

H $\quad-.99019 \quad-2.11344 \quad-2.39752$

$\mathrm{H} \quad-2.12561 \quad-.73616 \quad-2.13571$

H $\quad-.72479 \quad-.54149 \quad-3.22380$

C $\quad \begin{array}{llll}-3.96740 & 1.56622 & 2.13689\end{array}$

$\begin{array}{llll}\mathrm{H} & -4.35600 & 2.51185 & 1.74930\end{array}$

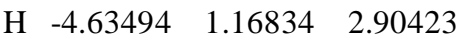

$\begin{array}{llll}\mathrm{H} & -2.96006 & 1.69114 & 2.53427\end{array}$

C $\quad-2.37844 \quad-5.86112 \quad 2.21042$

C $\quad-2.53325 \quad-4.79174 \quad 3.09846$

C $\quad-2.67063 \quad-3.49035 \quad 2.61561$

C $-2.65030 \quad-3.24062 \quad 1.23237$

$\begin{array}{llll}\text { C } & -2.48922 & -4.32120 & .34694\end{array}$

$\begin{array}{llll}\text { C } & -2.35857 & -5.62190 & .83091\end{array}$

H $\quad-2.28033 \quad-6.87570 \quad 2.58961$

\section{H $\quad-2.55583 \quad-4.97319 \quad 4.17053$ \\ H $-2.81103 \quad-2.65978 \quad 3.29874$ \\ $\mathrm{H}-2.48422 \quad-4.13841 \quad-.72611$ \\ $\begin{array}{llll}\mathrm{H} & -2.24886 & -6.45044 & .13520\end{array}$ \\ $\begin{array}{llll}\text { C } & .15128 & .04937 & 4.42057\end{array}$ \\ $\begin{array}{llll}\text { C } & -.27678 & .91061 & 3.40492\end{array}$ \\ $\begin{array}{llll}\text { C } & -.45660 & .44390 & 2.10330\end{array}$ \\ $\begin{array}{llll}\text { C } & -.18640 & -.90278 & 1.76153\end{array}$ \\ $\begin{array}{llll}\text { C } & .24531 & -1.75797 & 2.80270\end{array}$ \\ $\begin{array}{llll}\text { C } & .40332 & -1.29052 & 4.10665\end{array}$ \\ $\mathrm{H} \quad \begin{array}{llll}28495 & .41451 & 5.43614\end{array}$ \\ $\mathrm{H} \quad-.47959 \quad 1.95675 \quad 3.62850$ \\ $\begin{array}{llll}\mathrm{H} & -.80996 & 1.12590 & 1.33569\end{array}$ \\ $\begin{array}{llll}\mathrm{H} & .46005 & -2.79925 & 2.57458\end{array}$ \\ $\begin{array}{llll}\mathrm{H} & .73682 & -1.97714 & 4.88236\end{array}$ \\ Syn cisoid betaine}

$\mathrm{E}\left(\mathrm{B} 3 \mathrm{LYP} 6-31 \mathrm{G}^{*}\right)=-1661.877378$

$\mathrm{E}\left(\mathrm{B} 3 \mathrm{LYP} 6-311+\mathrm{G}^{* *}\right)=$

$-1662.179783$

$\mathrm{E}\left(\mathrm{B} 3 \mathrm{LYP} 6-31 \mathrm{G} *\left(\mathrm{CH}_{3} \mathrm{CN}\right)\right)=$ $-1661.945850$

$\mathrm{E}\left(\mathrm{B} 3 \mathrm{LYP} 6-311+\mathrm{G} * *\left(\mathrm{CH}_{3} \mathrm{CN}\right)\right)=$ $-1662.239056$

$\begin{array}{llll}\mathrm{C} & -1.61723 & -1.83126 & .40688 \\ \mathrm{~N} & -1.80615 & -2.81445 & -.65633 \\ \mathrm{H} & -1.77174 & -2.26670 & 1.40560 \\ \mathrm{H} & .01673 & -.92698 & -.68156 \\ \mathrm{~S} & -2.89991 & -3.93718 & -.39707 \\ \mathrm{O} & -2.70918 & -4.66399 & .90847 \\ \mathrm{O} & -4.32645 & -3.53242 & -.60419 \\ \mathrm{C} & -.12838 & -1.39415 & .29840 \\ \mathrm{~S} & .84624 & -3.01666 & .02146 \\ \mathrm{C} & 2.57758 & -2.51903 & .31812 \\ \mathrm{H} & 2.86026 & -1.81385 & -.46778 \\ \mathrm{H} & 2.70470 & -2.07137 & 1.30550 \\ \mathrm{H} & 3.17965 & -3.42729 & .22268 \\ \mathrm{C} & .53308 & -4.00896 & 1.51589 \\ \mathrm{H} & .75193 & -3.42662 & 2.41358 \\ \mathrm{H} & -.51083 & -4.33393 & 1.48644 \\ \mathrm{H} & 1.19606 & -4.87665 & 1.45697 \\ \mathrm{C} & -2.53852 & -5.12136 & -1.70387 \\ \mathrm{H} & -3.26091 & -5.93596 & -1.60389 \\ \mathrm{H} & -2.65672 & -4.63930 & -2.67625 \\ \mathrm{H} & -1.52096 & -5.49951 & -1.58807 \\ \mathrm{C} & 1.53315 & 1.22711 & 3.29908 \\ \mathrm{C} & 1.79010 & 1.43797 & 1.94156 \\ \mathrm{C} & 1.24890 & .57545 & .98778 \\ \mathrm{C} & .44178 & -.50839 & 1.37150 \\ \mathrm{C} & .18922 & -.71070 & 2.73939 \\ \mathrm{C} & .73206 & .15178 & 3.69387 \\ \mathrm{H} & 1.95086 & 1.89940 & 4.04452 \\ \mathrm{H} & 2.40666 & 2.27525 & 1.62375 \\ \mathrm{H} & 1.44287 & .74979 & -.06848 \\ \mathrm{H} & -.44748 & -1.52551 & 3.07075 \\ \mathrm{H} & .52077 & -.01417 & 4.74727 \\ \mathrm{C} & -4.09722 & 1.73386 & .15011 \\ \mathrm{C} & -3.58543 & 1.14566 & -1.01152 \\ \mathrm{C} & -2.79902 & -.00465 & -.92829 \\ \mathrm{C} & -2.50779 & -.58468 & .31334 \\ \mathrm{C} & -3.02871 & .00807 & 1.47029\end{array}$




\begin{abstract}
C $\quad-3.81894 \quad 1.15884 \quad 1.39240$
$\begin{array}{llll}\mathrm{H} & -4.71340 & 2.62809 & .08595\end{array}$

$\begin{array}{llll}\mathrm{H} & -3.80548 & 1.58018 & -1.98440\end{array}$

$\mathrm{H} \quad-2.42451 \quad-.47483 \quad-1.83293$

$\begin{array}{llll}\mathrm{H} & -2.82039 & -.43430 & 2.44215\end{array}$

H $\quad-4.21935 \quad 1.60249 \quad 2.30145$
\end{abstract}

Syn cisoid betaine (conformer 1)

$\mathrm{E}\left(\mathrm{B} 3 \mathrm{LYP} 6-31 \mathrm{G}^{*}\right)=-1661.873764$
$\mathrm{E}\left(\mathrm{B} 3 \mathrm{LYP} 6-311+\mathrm{G}^{* *}\right)=$
-1662.176403
$\mathrm{E}\left(\mathrm{B} 3 \mathrm{LYP} 6-31 \mathrm{G}^{*}\left(\mathrm{CH}_{3} \mathrm{CN}\right)\right)=$
-1661.942907
$\mathrm{E}\left(\mathrm{B} 3 \mathrm{LYP} 6-311+\mathrm{G}^{* *}\left(\mathrm{CH}_{3} \mathrm{CN}\right)\right)=$
-1662.237032

$\begin{array}{llll}\text { C } & -1.64900 & -1.77795 & .36924\end{array}$

$\begin{array}{llll}\mathrm{N} & -1.84757 & -2.74077 & -.70912\end{array}$

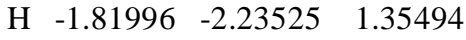

$\begin{array}{llll}\mathrm{H} & .03345 & -.89664 & -.68207\end{array}$

$\begin{array}{llll}\mathrm{S} & -2.95384 & -3.86089 & -.46512\end{array}$

$\begin{array}{llll}\text { O } & -3.04233 & -4.32931 & .95754\end{array}$

$\begin{array}{llll}\mathrm{O} & -4.29772 & -3.56141 & -1.05398\end{array}$

$\begin{array}{llll}\text { C } & -.14836 & -1.38741 & .27886\end{array}$

$\begin{array}{llll}\text { S } & .72957 & -3.07443 & .07710\end{array}$

C $1.05023 \quad-3.17231 \quad-1.71740$

H $\quad .06959 \quad-3.18260 \quad-2.19151$

H $\quad 1.64040 \quad-2.31043 \quad-2.03862$

H $\quad 1.59264 \quad-4.10324 \quad-1.90408$

$\begin{array}{llll}\text { C } & 2.43060 & -2.76191 & .66953\end{array}$

H $2.86483 \quad-1.88180 \quad .18966$

H $\quad 2.39092 \quad-2.63167 \quad 1.75224$

H $\quad 3.01130 \quad-3.65754 \quad .43056$

$\begin{array}{llll}\text { C } & -2.32599 & -5.24835 & -1.42863\end{array}$

H $-3.06382-6.05297 \quad-1.36593$

H $\quad-2.20210 \quad-4.93987 \quad-2.46870$

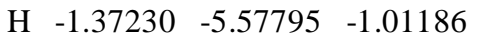

$\begin{array}{llll}\text { C } & 1.47195 & 1.06035 & 3.44055\end{array}$

$\begin{array}{llll}\text { C } & 1.67711 & 1.40072 & 2.10118\end{array}$

$\begin{array}{llll}\text { C } & 1.15980 & .59081 & 1.08807\end{array}$

$\begin{array}{llll}\text { C } & .42513 & -.56337 & 1.39938\end{array}$

$\begin{array}{llll}\text { C } & .22673 & -.89982 & 2.74905\end{array}$

$\begin{array}{llll}\text { C } & .74824 & -.09288 & 3.76121\end{array}$

$\mathrm{H} \quad 1.87233 \quad 1.69016 \quad 4.23131$

$\begin{array}{llll}\mathrm{H} & 2.23631 & 2.29680 & 1.84310\end{array}$

H $\quad 1.31246 \quad .86534 \quad .04641$

$\mathrm{H} \quad-.33826 \quad-1.78910 \quad 3.01763$

$\begin{array}{llll}\mathrm{H} & .58470 & -.36254 & 4.80173\end{array}$

$\begin{array}{llll}\text { C } & -4.02726 & 1.85869 & .18207\end{array}$

$\begin{array}{llll}\text { C } & -3.50230 & 1.29953 & -.98794\end{array}$

$\begin{array}{llll}\text { C } & -2.74831 & .12556 & -.92928\end{array}$

$\begin{array}{llll}\text { C } & -2.50418 & -.50760 & .29705\end{array}$

$\begin{array}{llll}\text { C } & -3.03624 & .05893 & 1.46287\end{array}$

C $\quad \begin{array}{llll}-3.79342 & 1.23287 & 1.40898\end{array}$

$\begin{array}{llll}\mathrm{H} & -4.61796 & 2.77111 & .13631\end{array}$

$\begin{array}{llll}\mathrm{H} & -3.68588 & 1.77602 & -1.94865\end{array}$

$\begin{array}{llll}\mathrm{H} & -2.35878 & -.31933 & -1.84048\end{array}$

$\mathrm{H} \quad-2.86016 \quad-.42264 \quad 2.42252$

H $\quad-4.20233 \quad 1.65560 \quad 2.32429$

Syn cisoid betaine (conformer 2)

$\mathrm{E}\left(\mathrm{B} 3 \mathrm{LYP} 6-31 \mathrm{G}^{*}\right)=-1661.865638$
$\mathrm{E}\left(\mathrm{B} 3 \mathrm{LYP} 6-311+\mathrm{G}^{* *}\right)=$
-1662.176920
$\mathrm{E}\left(\mathrm{B} 3 \mathrm{LYP} 6-31 \mathrm{G}^{*}\left(\mathrm{CH}_{3} \mathrm{CN}\right)\right)=$
-1661.943404
$\mathrm{E}\left(\mathrm{B} 3 \mathrm{LYP} 6-311+\mathrm{G}^{* *}\left(\mathrm{CH}_{3} \mathrm{CN}\right)\right)=$
-1662.23710

C $-1.61639-2.07042$

.60732

N $-1.73959-3.49589$

.33624

$\begin{array}{llll}\mathrm{H} & -1.92617 & -1.82894 & 1.63425\end{array}$

H $\quad .20951 \quad-1.88276 \quad-.56233$

$\begin{array}{llll}\text { S } & -2.90064 & -4.27095 & 1.10316\end{array}$

$\begin{array}{llll}\text { O } & -3.23618 & -3.68572 & 2.44258\end{array}$

$\begin{array}{llll}\text { O } & -4.11403 & -4.55301 & .27291\end{array}$

$\begin{array}{llll}\text { C } & -.09739 & -1.75665 & .48036\end{array}$

$\begin{array}{llll}\text { S } & .74487 & -3.22739 & 1.36193\end{array}$

$\begin{array}{llll}\text { C } & 1.20492 & -4.33548 & -.01448\end{array}$

$\mathrm{H} \quad \begin{array}{llll}26532 & -4.63802 & -.47546\end{array}$

$\begin{array}{llll}\mathrm{H} & 1.84436 & -3.80259 & -.72291\end{array}$

$\begin{array}{llll}\mathrm{H} & 1.73202 & -5.19261 & .41356\end{array}$

$\begin{array}{llll}\text { C } & 2.40078 & -2.58640 & 1.79824\end{array}$

$\begin{array}{llll}\mathrm{H} & 2.89520 & -2.13476 & .93485\end{array}$

$\begin{array}{llll}\mathrm{H} & 2.27760 & -1.85701 & 2.60039\end{array}$

$\begin{array}{llll}\mathrm{H} & 2.97494 & -3.44115 & 2.16752\end{array}$

$\begin{array}{llll}\text { C } & -2.17392 & -5.89115 & 1.41507\end{array}$

$\begin{array}{llll}\mathrm{H} & -2.94529 & -6.51632 & 1.87325\end{array}$

$\begin{array}{llll}\mathrm{H} & -1.85502 & -6.33258 & .46859\end{array}$

$\begin{array}{llll}\mathrm{H} & -1.32417 & -5.78266 & 2.09207\end{array}$

$\begin{array}{llll}\text { C } & 1.22456 & 2.10121 & 1.89186\end{array}$

$\begin{array}{llll}\text { C } & 1.56994 & 1.65454 & .61417\end{array}$

$\begin{array}{llll}\text { C } & 1.15018 & .39680 & .17621\end{array}$

$\begin{array}{llll}\text { C } & .37262 & -.42601 & 1.00420\end{array}$

$\begin{array}{llll}\text { C } & .03247 & .03013 & 2.28967\end{array}$

$\begin{array}{llll}\text { C } & .45759 & 1.28457 & 2.72951\end{array}$

$\begin{array}{llll}\mathrm{H} & 1.54986 & 3.08082 & 2.23376\end{array}$

$\begin{array}{llll}\mathrm{H} & 2.16335 & 2.28421 & -.04432\end{array}$

$\begin{array}{llll}\mathrm{H} & 1.41272 & .05738 & -.82345\end{array}$

$\begin{array}{llll}\mathrm{H} & -.56840 & -.58844 & 2.95171\end{array}$

$\begin{array}{llll}\mathrm{H} & .18463 & 1.62605 & 3.72502\end{array}$

$\begin{array}{llll}\text { C } & -3.74521 & .61431 & -2.07268\end{array}$

$\begin{array}{llll}\text { C } & -3.18919 & -.57318 & -2.56120\end{array}$

$\begin{array}{llll}\text { C } & -2.51792 & -1.44198 & -1.69810\end{array}$

$\begin{array}{llll}\text { C } & -2.38863 & -1.13890 & -.33608\end{array}$

$\begin{array}{llll}\text { C } & -2.95264 & .05028 & .14471\end{array}$

$\begin{array}{llll}\text { C } & -3.62702 & .92236 & -.71490\end{array}$

$\begin{array}{llll}\mathrm{H} & -4.27148 & 1.28989 & -2.74371\end{array}$

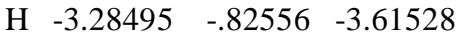

$\begin{array}{llll}\mathrm{H} & -2.10516 & -2.37461 & -2.07132\end{array}$

$\begin{array}{llll}\mathrm{H} & -2.86670 & .39708 & 1.20082\end{array}$

$\begin{array}{llll}\mathrm{H} & -4.06279 & 1.83917 & -.32335\end{array}$

Syn cisoid betaine (conformer 3)

E(B3LYP 6-31G*) $=-1661.864107$

$\mathrm{E}\left(\mathrm{B} 3 \mathrm{LYP} 6-311+\mathrm{G}^{* *}\right)=$

$-1662.183690$

$\mathrm{E}\left(\mathrm{B} 3 \mathrm{LYP} 6-31 \mathrm{G}^{*}\left(\mathrm{CH}_{3} \mathrm{CN}\right)\right)=$

$-1661.944963$

$\mathrm{E}\left(\mathrm{B} 3 \mathrm{~L}\right.$ YP $\left.6-311+\mathrm{G}^{* * *}\left(\mathrm{CH}_{3} \mathrm{CN}\right)\right)=$

$-1662.237562$

$\begin{array}{llll}\mathrm{C} & -1.65039 & -1.74617 & .42571 \\ \mathrm{~N} & -1.86090 & -3.06517 & 1.00760 \\ \mathrm{H} & -2.12291 & -.95804 & 1.02718 \\ \mathrm{H} & .22802 & -.98894 & -.45015 \\ \mathrm{~S} & -3.17696 & -3.28217 & 1.86724 \\ \mathrm{O} & -2.99626 & -4.51641 & 2.68218 \\ \mathrm{O} & -3.60008 & -2.06909 & 2.63701 \\ \mathrm{C} & -.11457 & -1.42872 & .49216 \\ \mathrm{~S} & .78035 & -3.11260 & .42854 \\ \mathrm{C} & 2.48603 & -2.60058 & .03385 \\ \mathrm{H} & 2.48770 & -2.19813 & -.98226 \\ \mathrm{H} & 2.85021 & -1.85883 & .74857 \\ \mathrm{H} & 3.10392 & -3.50201 & .06835 \\ \mathrm{C} & .99811 & -3.67441 & 2.14791 \\ \mathrm{H} & 1.40430 & -2.86878 & 2.76253 \\ \mathrm{H} & .01317 & -3.98512 & 2.49563 \\ \mathrm{H} & 1.68958 & -4.52162 & 2.11512 \\ \mathrm{C} & -4.59002 & -3.64749 & .78881 \\ \mathrm{H} & -5.46714 & -3.82135 & 1.41874 \\ \mathrm{H} & -4.76683 & -2.79757 & .12580 \\ \mathrm{H} & -4.36472 & -4.54146 & .20263 \\ \mathrm{C} & 1.20759 & 1.12163 & 3.71937 \\ \mathrm{C} & 1.76993 & 1.23920 & 2.44569 \\ \mathrm{C} & 1.34143 & .40182 & 1.41305 \\ \mathrm{C} & .34449 & -.56245 & 1.63814 \\ \mathrm{C} & -.22126 & -.66807 & 2.92116 \\ \mathrm{C} & .21091 & .16830 & 3.95186 \\ \mathrm{H} & 1.53787 & 1.77401 & 4.52414 \\ \mathrm{H} & 2.53534 & 1.98652 & 2.25132 \\ \mathrm{H} & 1.76922 & .51359 & .41895 \\ \mathrm{H} & -1.01456 & -1.38611 & 3.10512 \\ \mathrm{H} & -.23794 & .07937 & 4.93812 \\ \mathrm{C} & -3.26107 & -1.26569 & -3.58384 \\ \mathrm{C} & -2.89598 & -2.53532 & -3.12702 \\ \mathrm{C} & -2.36850 & -2.69451 & -1.84266 \\ \mathrm{C} & -2.19418 & -1.59082 & -.99944 \\ \mathrm{C} & -2.56239 & -.32067 & -1.46648 \\ \mathrm{C} & -3.09229 & -.15618 & -2.74866 \\ \mathrm{H} & -3.68320 & -1.14206 & -4.57855 \\ \mathrm{H} & -3.03216 & -3.40530 & -3.76622 \\ \mathrm{H} & -2.11278 & -3.688178 & -1.47116 \\ & -3.38601 & .83373 & -3.09083\end{array}$

\section{Anti cisoid betaine}

E(B3LYP 6-31G*) =

$-1661.882336$

$\mathrm{E}\left(\mathrm{B} 3 \mathrm{LYP} 6-311+\mathrm{G}^{* *}\right)=$

$-1662.181739$

$\mathrm{E}\left(\mathrm{B} 3 \mathrm{LYP} 6-31 \mathrm{G} *\left(\mathrm{CH}_{3} \mathrm{CN}\right)\right)=$

$-1661.945894$

$\mathrm{E}\left(\mathrm{B} 3 \mathrm{LYP} 6-311+\mathrm{G}^{* *}\left(\mathrm{CH}_{3} \mathrm{CN}\right)\right)=$

$-1662.238912$

$\begin{array}{llll}\text { C C } & -1.76404 & -1.61149 & .50124\end{array}$

$\begin{array}{llll}\mathrm{N} & -2.46292 & -.37625 & .84234\end{array}$

$\begin{array}{llll}\mathrm{H} & -1.99806 & -1.93394 & -.52456\end{array}$

$\mathrm{H} \quad .28041 \quad-2.08630 \quad-.09170$

$\begin{array}{llll}\text { S } & -3.92629 & -.22545 & .24355\end{array}$

$\begin{array}{llll}\text { O } & -4.35447 & 1.19284 & .39378\end{array}$

$\begin{array}{llll}\text { O } & -4.05941 & -.77631 & -1.14820\end{array}$

$\begin{array}{llll}\text { C } & -.23254 & -1.31547 & .49263\end{array}$ 


$\begin{array}{llll}\mathrm{S} & -.01561 & .28881 & -.47769 \\ \mathrm{C} & 1.76230 & .21707 & -.89462 \\ \mathrm{H} & 2.32444 & .31036 & .03691 \\ \mathrm{H} & 2.00618 & -.71834 & -1.40492 \\ \mathrm{H} & 1.97646 & 1.07511 & -1.53779 \\ \mathrm{C} & -.73944 & -.05474 & -2.11616 \\ \mathrm{H} & -.33976 & -.98782 & -2.52202 \\ \mathrm{H} & -1.82406 & -.10423 & -1.99739 \\ \mathrm{H} & -.47058 & .78904 & -2.75824 \\ \mathrm{C} & -5.14083 & -1.16627 & 1.20974 \\ \mathrm{H} & -6.12958 & -.97477 & .78335 \\ \mathrm{H} & -4.90519 & -2.23042 & 1.15548 \\ \mathrm{H} & -5.10670 & -.82818 & 2.24750 \\ \mathrm{C} & -2.76856 & -5.03973 & 2.97578 \\ \mathrm{C} & -2.75263 & -3.75701 & 3.53314 \\ \mathrm{C} & -2.41160 & -2.65314 & 2.75004 \\ \mathrm{C} & -2.08024 & -2.81460 & 1.39785 \\ \mathrm{C} & -2.09594 & -4.10306 & .84813 \\ \mathrm{C} & -2.43773 & -5.21095 & 1.62888 \\ \mathrm{H} & -3.04764 & -5.89741 & 3.58370 \\ \mathrm{H} & -3.01815 & -3.61381 & 4.57839 \\ \mathrm{H} & -2.42805 & -1.65443 & 3.17503 \\ \mathrm{H} & -1.86054 & -4.23877 & -.20676 \\ \mathrm{H} & -2.45979 & -6.20235 & 1.18159 \\ \mathrm{C} & 1.82319 & -.85718 & 4.26462 \\ \mathrm{C} & .72379 & -.04295 & 3.97295 \\ \mathrm{C} & .05281 & -.17645 & 2.75687 \\ \mathrm{C} & .47539 & -1.13038 & 1.81624 \\ \mathrm{C} & 1.58377 & -1.93712 & 2.11192 \\ \mathrm{C} & 2.25140 & -1.80549 & 3.33194 \\ \mathrm{H} & 2.34360 & -.75193 & 5.21375 \\ \mathrm{H} & .38515 & .69635 & 4.69511 \\ \mathrm{H} & -.81347 & .43592 & 2.53274 \\ \mathrm{H} & 1.91890 & -2.67879 & 1.38993 \\ \mathrm{H} & 3.10449 & -2.44319 & 3.55087\end{array}$

\section{Anti cisoid betaine (conformer)}

\begin{tabular}{|c|c|c|c|}
\hline \multicolumn{4}{|c|}{$\begin{array}{l}\mathrm{E}\left(\mathrm{B} 3 \mathrm{LYP} 6-31 \mathrm{G}^{*}\left(\mathrm{CH}_{3} \mathrm{CN}\right)\right)= \\
-1661.939353 \\
\mathrm{E}\left(\mathrm{B} 3 \mathrm{LYP} 6-311+\mathrm{G}^{* *}\left(\mathrm{CH}_{3} \mathrm{CN}\right)\right)= \\
-1662.233969\end{array}$} \\
\hline C & -1.77243 & -1.68549 & .50274 \\
\hline $\mathrm{N}$ & -2.36882 & -.35757 & \\
\hline I & -2.03132 & -2.22597 & -.42114 \\
\hline I & -.09374 & -1.04287 & -.67364 \\
\hline & -3.85958 & -.19396 & \\
\hline ) & -4.09290 & 1.25334 & -.23760 \\
\hline 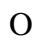 & -4.21657 & -1.11764 & -1.09064 \\
\hline & -.23705 & -1.43388 & .33708 \\
\hline & .20080 & .08693 & 1.41279 \\
\hline & 2.01827 & -.01664 & 1.56412 \\
\hline$H$ & 2.26255 & -.82143 & 2.25828 \\
\hline & 2.48130 & -.19245 & \\
\hline & 2.34667 & .94006 & 1.97974 \\
\hline & .09258 & 1.48115 & .23809 \\
\hline 1 & .71017 & 1.27391 & -.64009 \\
\hline & -.96098 & 1.56720 & -.02524 \\
\hline
\end{tabular}

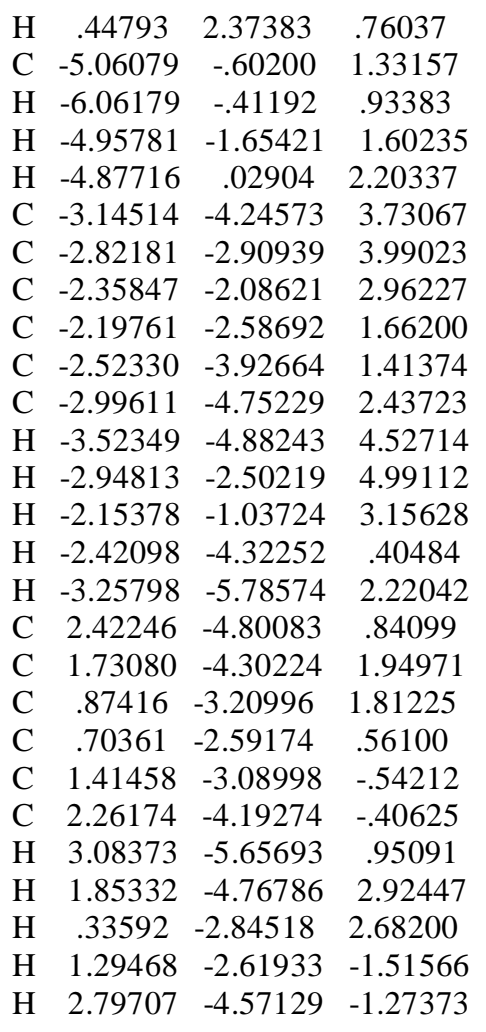

\section{Syn Torsional TS}

$\mathrm{E}(\mathrm{B} 3 L Y P$ 6-31G* $)=-1661.881304$
$\mathrm{E}(\mathrm{B} 3 \mathrm{G}$ YP 6-311+G**)
-1662.174845
$\mathrm{E}\left(\mathrm{B} 3 \mathrm{LYP} 6-31 \mathrm{G}^{*}\left(\mathrm{CH}_{3} \mathrm{CN}\right)\right)=$
-1661.926216
$\mathrm{E}\left(\mathrm{B} 3 \mathrm{LYP} 6-311+\mathrm{G}^{* *}\left(\mathrm{CH}_{3} \mathrm{CN}\right)\right)=$
-1662.220129

$\begin{array}{cccc}\mathrm{C} & -1.67008 & -1.63319 & .261380 \\ \mathrm{~N} & -2.37121 & -.35849 & .332849 \\ \mathrm{H} & -1.82297 & -2.12671 & -.710106 \\ \mathrm{H} & .05609 & -.34690 & .499105 \\ \mathrm{~S} & -2.16921 & .68687 & -.832836 \\ \mathrm{O} & -.82627 & 1.37715 & -.859826 \\ \mathrm{O} & -2.52879 & .18687 & -2.210353 \\ \mathrm{C} & -.08137 & -1.42715 & .444869 \\ \mathrm{~S} & .95605 & -1.87796 & -1.098039 \\ \mathrm{C} & -.09600 & -1.89861 & -2.589283 \\ \mathrm{H} & -.62983 & -2.85095 & -2.601296 \\ \mathrm{H} & -.79145 & -1.05544 & -2.613125 \\ \mathrm{H} & .59812 & -1.86834 & -3.434946 \\ \mathrm{C} & 1.91531 & -.34572 & -1.339113 \\ \mathrm{H} & 1.22069 & .48871 & -1.456764 \\ \mathrm{H} & 2.54153 & -.21850 & -.452705 \\ \mathrm{H} & 2.54703 & -.48687 & -2.219911 \\ \mathrm{C} & -3.37411 & 1.95711 & -.417179 \\ \mathrm{H} & -3.25745 & 2.75954 & -1.150322 \\ \mathrm{H} & -4.38059 & 1.53831 & -.474383 \\ \mathrm{H} & -3.17353 & 2.33146 & .587977 \\ \mathrm{C} & 1.83227 & -3.17384 & 3.899180 \\ \mathrm{C} & 1.66133 & -1.79203 & 3.796826 \\ \mathrm{C} & 1.06506 & -1.24505 & 2.657722 \\ \mathrm{C} & .61812 & -2.07261 & 1.617130\end{array}$

$\begin{array}{lcll}\mathrm{C} & .80126 & -3.46063 & 1.726366 \\ \mathrm{C} & 1.40494 & -4.00608 & 2.858100 \\ \mathrm{H} & 2.29989 & -3.60247 & 4.782365 \\ \mathrm{H} & 1.99428 & -1.13642 & 4.597811 \\ \mathrm{H} & .93423 & -.16840 & 2.581349 \\ \mathrm{H} & .46534 & -4.11798 & .928416 \\ \mathrm{H} & 1.54086 & -5.08219 & 2.930854 \\ \mathrm{C} & -3.38364 & -4.31996 & 3.205201 \\ \mathrm{C} & -3.09180 & -2.99530 & 3.549342 \\ \mathrm{C} & -2.53671 & -2.12993 & 2.606590 \\ \mathrm{C} & -2.26119 & -2.57345 & 1.305959 \\ \mathrm{C} & -2.55979 & -3.89844 & .968701 \\ \mathrm{C} & -3.11800 & -4.76940 & 1.910235 \\ \mathrm{H} & -3.82372 & -4.99216 & 3.938507 \\ \mathrm{H} & -3.30503 & -2.63373 & 4.553002 \\ \mathrm{H} & -2.33310 & -1.09566 & 2.866122 \\ \mathrm{H} & -2.37059 & -4.25224 & -.043711 \\ \mathrm{H} & -3.35409 & -5.79280 & 1.627034\end{array}$

\section{Anti Torsional TS}

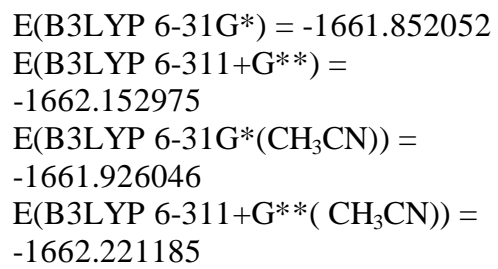

$\begin{array}{llll}\text { C } & -1.94909 & -1.81576 & .70620\end{array}$

$\begin{array}{llll}\mathrm{N} & -2.63186 & -.57666 & 1.00385\end{array}$

$\mathrm{H} \quad-2.28792 \quad-2.24971 \quad-.24260$

$\begin{array}{llll}\mathrm{H} & -.28609 & -.42293 & .55569\end{array}$

$\begin{array}{llll}\mathrm{S} & -3.45434 & .11781 & -.15875\end{array}$

$\begin{array}{lllll}\mathrm{O} & -3.77464 & -.77937 & -1.31382\end{array}$

$\begin{array}{llll}\text { O } & -4.62529 & .82744 & .43237\end{array}$

$\begin{array}{llll}\text { C } & -.39131 & -1.50634 & .45738\end{array}$

$\begin{array}{llll}\text { S } & .74949 & -2.14915 & 1.82183\end{array}$

$\begin{array}{llll}\text { C } & 2.38100 & -1.56515 & 1.24871\end{array}$

H $\quad 2.66678 \quad-2.17004 \quad .38696$

$\begin{array}{llll}\mathrm{H} & 2.33477 & -.50620 & .98319\end{array}$

H $3.08600 \quad-1.72745 \quad 2.06853$

$\begin{array}{llll}\text { C } & .46261 & -.98957 & 3.20221\end{array}$

$\mathrm{H} \quad .50540 \quad .04218 \quad 2.84476$

$\mathrm{H} \quad-.51394 \quad-1.21346 \quad 3.63053$

H $\quad 1.24504 \quad-1.180913 .94164$

$\begin{array}{llll}\text { C } & -2.48235 & 1.46454 & -.88773\end{array}$

H $\quad-3.11339 \quad 1.99740 \quad-1.60450$

$\begin{array}{llll}\mathrm{H} & -2.16490 & 2.14668 & -.09565\end{array}$

$\begin{array}{llll}\mathrm{H} & -1.61343 & 1.04803 & -1.40059\end{array}$

$\begin{array}{llll}\text { C } & -2.75258 & -4.83571 & 3.74908\end{array}$

$\begin{array}{llll}\text { C } & -2.88766 & -3.47844 & 4.05355\end{array}$

$\begin{array}{llll}\text { C } & -2.63523 & -2.50847 & 3.07894\end{array}$

$\begin{array}{llll}\text { C } & -2.22315 & -2.87329 & 1.78909\end{array}$

$\begin{array}{llll}\text { C } & -2.11741 & -4.24077 & 1.48872\end{array}$

$\begin{array}{llll}\text { C } & -2.37599 & -5.21417 & 2.45739\end{array}$

$\mathrm{H} \quad-2.95678 \quad-5.59155 \quad 4.50409$

H $\quad-3.20486 \quad-3.17158 \quad 5.04791$

H $-2.79596-1.45789 \quad 3.29944$

$\begin{array}{llll}\mathrm{H} & -1.86484 & -4.55403 & .47855\end{array}$

H $\quad-2.29665 \quad-6.26756 \quad 2.19772$

C $\quad .82772 \quad-2.62153 \quad-3.53192$

C $\quad .82665 \quad-1.28323 \quad-3.13308$ 


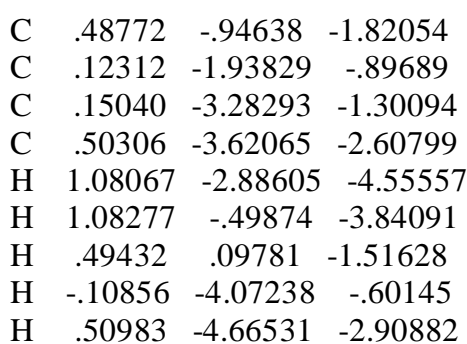

\section{Syn transoid betaine}

$\mathrm{E}\left(\mathrm{B} 3 \mathrm{LYP} 6-31 \mathrm{G}^{*}\right)=-1661.843896$ $\mathrm{E}\left(\mathrm{B} 3 \mathrm{LYP} 6-311+\mathrm{G}^{* *}\right)=$ $-1662.169943$

$\mathrm{E}\left(\mathrm{B} 3 \mathrm{LYP} 6-31 \mathrm{G} *\left(\mathrm{CH}_{3} \mathrm{CN}\right)\right)=$ $-1661.934037$

$\mathrm{E}\left(\mathrm{B} 3 \mathrm{LYP} 6-311+\mathrm{G}^{* *}\left(\mathrm{CH}_{3} \mathrm{CN}\right)\right)=$ $-1662.227545$

$\begin{array}{lcll}\mathrm{C} & -1.68416 & -1.62722 & .31444 \\ \mathrm{~N} & -2.36820 & -.34547 & .36306 \\ \mathrm{H} & -1.85624 & -2.15306 & -.63996 \\ \mathrm{H} & .13037 & -.76819 & -.49904 \\ \mathrm{~S} & -2.38357 & .49692 & -.97715 \\ \mathrm{O} & -1.07203 & 1.13386 & -1.36634 \\ \mathrm{O} & -3.00479 & -.20366 & -2.15591 \\ \mathrm{C} & -.12044 & -1.35861 & .38635 \\ \mathrm{~S} & .87247 & -2.95706 & .16062 \\ \mathrm{C} & .51577 & -3.43302 & -1.56399 \\ \mathrm{H} & -.51458 & -3.78861 & -1.61454 \\ \mathrm{H} & .66594 & -2.57928 & -2.22917 \\ \mathrm{H} & 1.19862 & -4.24771 & -1.81983 \\ \mathrm{C} & 2.58313 & -2.34426 & -.00798 \\ \mathrm{H} & 2.63045 & -1.55807 & -.76528 \\ \mathrm{H} & 2.88871 & -1.96448 & .96789 \\ \mathrm{H} & 3.20755 & -3.19662 & -.28861 \\ \mathrm{C} & -3.47481 & 1.85827 & -.53801 \\ \mathrm{H} & -3.53109 & 2.51875 & -1.40731 \\ \mathrm{H} & -4.46530 & 1.47197 & -.29285 \\ \mathrm{H} & -3.05497 & 2.39361 & .31528 \\ \mathrm{C} & 1.32000 & .78475 & 3.85236 \\ \mathrm{C} & 1.16498 & 1.42622 & 2.62011 \\ \mathrm{C} & .71270 & .71172 & 1.51023 \\ \mathrm{C} & .39745 & -.65135 & 1.61955 \\ \mathrm{C} & .55398 & -1.28961 & 2.86031 \\ \mathrm{C} & 1.01692 & -.57445 & 3.96778 \\ \mathrm{H} & 1.67398 & 1.34116 & 4.71701 \\ \mathrm{H} & 1.39494 & 2.48453 & 2.52094 \\ \mathrm{H} & .57066 & 1.21110 & .55656 \\ \mathrm{H} & .30726 & -2.34161 & 2.97434 \\ \mathrm{H} & 1.13253 & -1.08048 & 4.92298 \\ \mathrm{C} & -3.20770 & -4.20227 & 3.46155 \\ \mathrm{C} & -3.11817 & -2.82080 & 3.66253 \\ \mathrm{C} & -2.62234 & -1.99312 & 2.65438 \\ \mathrm{C} & -2.20238 & -2.53031 & 1.42916 \\ \mathrm{C} & -2.30512 & -3.91445 & 1.23383 \\ \mathrm{C} & -2.80145 & -4.74805 & 2.24174 \\ \mathrm{H} & -3.60453 & -4.84512 & 4.24390 \\ \mathrm{H} & -3.44647 & -2.38452 & 4.60326 \\ \mathrm{H} & -2.58197 & -.91867 & 2.79427 \\ \mathrm{H} & -2.03363 & -4.34953 & .27300 \\ \mathrm{H} & -2.88636 & -5.81792 & 2.06554 \\ & & & \end{array}$

Syn transoid betaine (conformer 1)

$\mathrm{E}($ B3LYP 6-31G*) $=-1661.823887$

$\mathrm{E}($ B3LYP 6-311+G**) $=$

$-1662.170099$

$\mathrm{E}\left(\mathrm{B} 3 \mathrm{LYP} 6-31 \mathrm{G} *\left(\mathrm{CH}_{3} \mathrm{CN}\right)\right)=$ $-1661.933977$

$\mathrm{E}\left(\mathrm{B} 3 \mathrm{LYP} 6-311+\mathrm{G}^{* *}\left(\mathrm{CH}_{3} \mathrm{CN}\right)\right)=$ $-1662.227627$

$\begin{array}{llll}\mathrm{C} & -2.13191 & -1.89187 & .75372 \\ \mathrm{~N} & -2.93057 & -.68609 & .90411 \\ \mathrm{H} & -2.38700 & -2.44224 & -.16779 \\ \mathrm{H} & -.53867 & -.85929 & -.29668\end{array}$

S -3.18916

$\begin{array}{llll}\mathrm{O} & -2.01385 & .96785 & -.90891\end{array}$

$\begin{array}{llll}\mathrm{O} & -3.84286 & -.58413 & -1.53309\end{array}$

$\begin{array}{llll}\text { C } & -.60600 & -1.47517 & .60406\end{array}$

$\begin{array}{llll}\mathrm{S} & .48227 & -2.97733 & .21186\end{array}$

$\begin{array}{llll}\text { C } & -.08830 & -3.50647 & -1.43885\end{array}$

H $\quad-1.06583 \quad-3.97846 \quad-1.33077$

$\mathrm{H} \quad-.14063 \quad-2.64951 \quad-2.11499$

H $\quad .63200 \quad-4.24388-1.80333$

$\begin{array}{llll}\text { C } & 2.07946 & -2.20817 & -.22114\end{array}$

H $\quad 1.93455 \quad-1.43698 \quad-.98142$

H $\quad 2.49530 \quad-1.78220 \quad .69311$

$\begin{array}{llll}\mathrm{H} & 2.72981 & -3.00457 & -.59209\end{array}$

$\begin{array}{llll}\text { C } & -4.38318 & 1.37961 & .18098\end{array}$

$\begin{array}{llll}\mathrm{H} & -4.60797 & 2.04358 & -.65815\end{array}$

$\begin{array}{llll}\mathrm{H} & -5.29077 & .87409 & .51452\end{array}$

$\begin{array}{llll}\mathrm{H} & -3.94256 & 1.94774 & 1.00168\end{array}$

$\begin{array}{llll}\text { C } & 1.14838 & .75816 & 3.86496\end{array}$

$\begin{array}{llll}\text { C } & .75187 & 1.39502 & 2.68562\end{array}$

$\begin{array}{llll}\text { C } & .19834 & .65423 & 1.64032\end{array}$

$\begin{array}{llll}\text { C } & .02282 & -.73275 & 1.76378\end{array}$

$\begin{array}{llll}\text { C } & .42230 & -1.36620 & 2.95180\end{array}$

C $\quad .98493 \quad-.62374 \quad 3.99320$

$\mathrm{H} \quad 1.58155 \quad 1.33528 \quad 4.67864$

$\begin{array}{llll}\mathrm{H} & .87213 & 2.47057 & 2.57730\end{array}$

$\begin{array}{llll}\mathrm{H} & -.13164 & 1.14878 & .73169\end{array}$

$\mathrm{H} \quad .28970 \quad-2.43723 \quad 3.07695$

H $\quad 1.28872 \quad-1.12712 \quad 4.90777$

C $-2.91496 \quad-4.56090 \quad 4.08824$

$\begin{array}{llll}\text { C } & -2.94086 & -3.17442 & 4.27318\end{array}$

C $\quad-2.68282 \quad-2.31645 \quad 3.20323$

$\begin{array}{llll}\text { C } & -2.38970 & -2.82717 & 1.93070\end{array}$

C $-2.37643 \quad-4.21732 \quad 1.75301$

$\begin{array}{llll}\text { C } & -2.63382 & -5.08119 & 2.82294\end{array}$

$\mathrm{H} \quad-3.12675 \quad-5.22876 \quad 4.92015$

H $-3.17351 \quad-2.75923 \quad 5.25129$

H $-2.73164 \quad-1.24122 \quad 3.33509$

H $-2.20189-4.63845 \quad .76394$

H $\quad-2.63175 \quad-6.15677 \quad 2.66175$

Syn transoid betaine (conformer 2)

$\mathrm{E}($ B3LYP 6-31G*) $=-1661.827783$

$\mathrm{E}\left(\mathrm{B} 3 \mathrm{LYP} 6-311+\mathrm{G}^{* *}\right)=$ $-1662.157149$
$\mathrm{E}\left(\mathrm{B} 3 \mathrm{LYP} 6-31 \mathrm{G} *\left(\mathrm{CH}_{3} \mathrm{CN}\right)\right)=$ $-1661.933685$

$\mathrm{E}\left(\mathrm{B} 3 \mathrm{LYP} 6-311+\mathrm{G} * *\left(\mathrm{CH}_{3} \mathrm{CN}\right)\right)=$ $-1662.231583$

$\begin{array}{llll}\text { C } & -1.13721 & -1.50450 & .16197\end{array}$

$\begin{array}{llll}\mathrm{N} & -1.77604 & -.32195 & -.41007\end{array}$

$\mathrm{H} \quad-1.03961 \quad-2.29416 \quad-.60155$

$\begin{array}{llll}\mathrm{H} & .58650 & -.27203 & -.22087\end{array}$

$\begin{array}{llll}\mathrm{S} & -2.97880 & -.59198 & -1.40906\end{array}$

$\begin{array}{llll}\mathrm{O} & -3.22183 & .65873 & -2.18592\end{array}$

$\begin{array}{llll}\mathrm{O} & -2.81002 & -1.83012 & -2.23647\end{array}$

$\begin{array}{llll}\text { C } & .32809 & -1.03400 & .51816\end{array}$

$\begin{array}{llll}\text { S } & 1.50083 & -2.46610 & .11017\end{array}$

C $\quad 1.61444 \quad-2.34907 \quad-1.70802$

H $\quad .61464 \quad-2.49926 \quad-2.12035$

H $\quad 2.00566 \quad-1.36879 \quad-1.99099$

H $\quad 2.27874 \quad-3.14839-2.04622$

$\begin{array}{llll}\text { C } & 3.14501 & -1.83971 & .58912\end{array}$

$\begin{array}{llll}\mathrm{H} & 3.29452 & -.83135 & .19518\end{array}$

$\begin{array}{llll}\mathrm{H} & 3.20013 & -1.84915 & 1.67860\end{array}$

$\begin{array}{llll}\mathrm{H} & 3.88116 & -2.53330 & .17369\end{array}$

$\begin{array}{llll}\text { C } & -4.54783 & -.84620 & -.53249\end{array}$

H $\quad-5.33670 \quad-.93737 \quad-1.28468$

$\begin{array}{llll}\mathrm{H} & -4.49314 & -1.75416 & .07003\end{array}$

$\begin{array}{llll}\mathrm{H} & -4.73942 & .01840 & .10741\end{array}$

C $\quad 1.20543 \quad .54180 \quad 4.44496$

$\begin{array}{llll}\text { C } & 1.24598 & 1.36534 & 3.31737\end{array}$

$\begin{array}{llll}\text { C } & .97272 & .83537 & 2.05375\end{array}$

$\begin{array}{llll}\text { C } & .63373 & -.51821 & 1.90425\end{array}$

$\begin{array}{llll}\text { C } & .60137 & -1.34022 & 3.04405\end{array}$

$\begin{array}{llll}\text { C } & .88997 & -.81331 & 4.30324\end{array}$

$\begin{array}{llll}\mathrm{H} & 1.42219 & .95116 & 5.42889\end{array}$

H $\quad 1.49666 \quad 2.41895 \quad 3.41686$

H $1.01375 \quad 1.47942 \quad 1.17869$

$\begin{array}{llll}\mathrm{H} & .34380 & -2.39204 & 2.95454\end{array}$

H $\quad .86076 \quad-1.46190 \quad 5.17532$

C $\quad-3.63164 \quad-3.25324 \quad 3.25308$

$\begin{array}{llll}\text { C } & -3.39615 & -1.87346 & 3.23742\end{array}$

C $\quad-2.56054-1.31616 \quad 2.27154$

$\begin{array}{llll}\text { C } & -1.93864 & -2.12512 & 1.30729\end{array}$

$\begin{array}{llll}\text { C } & -2.17266 & -3.50472 & 1.33754\end{array}$

$\begin{array}{llll}\text { C } & -3.01532 & -4.06888 & 2.30230\end{array}$

$\mathrm{H} \quad-4.30061 \quad-3.68534 \quad 3.99396$

$\mathrm{H} \quad-3.87752 \quad-1.22909 \quad 3.97009$

$\begin{array}{llll}\mathrm{H} & -2.41014 & -.24144 & 2.23723\end{array}$

$\begin{array}{llll}\mathrm{H} & -1.71810 & -4.14183 & .58050\end{array}$

H $\quad-3.20258 \quad-5.14036 \quad 2.29680$

\section{Syn transoid betaine (conformer 3)}

$\mathrm{E}\left(\mathrm{B} 3 \mathrm{LYP} 6-31 \mathrm{G}^{*}\right)=-1661.807630$

$\mathrm{E}\left(\mathrm{B} 3 \mathrm{LYP} 6-311+\mathrm{G}^{* *}\right)=$

$-1662.169799$

$\mathrm{E}\left(\mathrm{B} 3 \mathrm{LYP} 6-31 \mathrm{G}^{*}\left(\mathrm{CH}_{3} \mathrm{CN}\right)\right)=$

$-1661.933964$

$\mathrm{E}\left(\mathrm{B} 3 \mathrm{LYP} 6-311+\mathrm{G}^{* *}\left(\mathrm{CH}_{3} \mathrm{CN}\right)\right)=$ $-1662.227539$

$\begin{array}{llll}\text { C } & -2.20631 & -2.04626 & .82589\end{array}$

$\begin{array}{llll}\mathrm{N} & -2.96932 & -.81219 & .75331\end{array}$ 


$\begin{array}{cccc}\text { H } & -2.47948 & -2.74966 & .02250 \\ \mathrm{H} & -.59640 & -1.28526 & -.41164 \\ \mathrm{~S} & -3.25303 & -.23562 & -.69263 \\ \mathrm{O} & -2.07247 & .39443 & -1.38933 \\ \mathrm{O} & -3.97556 & -1.17728 & -1.61681 \\ \mathrm{C} & -.67145 & -1.70398 & .59552 \\ \mathrm{~S} & .38850 & -3.27341 & .51439 \\ \mathrm{C} & -.17834 & -4.10092 & -1.00974 \\ \mathrm{H} & -1.16555 & -4.52727 & -.82521 \\ \mathrm{H} & -.20930 & -3.39025 & -1.83925 \\ \mathrm{H} & .53228 & -4.90535 & -1.21668 \\ \mathrm{C} & 2.00630 & -2.63460 & -.03739 \\ \mathrm{H} & 1.88795 & -2.02576 & -.93693 \\ \mathrm{H} & 2.41947 & -2.04470 & .78192 \\ \mathrm{H} & 2.64378 & -3.50084 & -.23274 \\ \mathrm{C} & -4.38913 & 1.11252 & -.33229 \\ \mathrm{H} & -4.62708 & 1.59778 & -1.28267 \\ \mathrm{H} & -5.29618 & .71505 & .12581 \\ \mathrm{H} & -3.90452 & 1.82093 & .34153 \\ \mathrm{C} & 1.11013 & 1.12246 & 3.33962 \\ \mathrm{C} & .72644 & 1.50991 & 2.05252 \\ \mathrm{C} & .16692 & .57785 & 1.17761 \\ \mathrm{C} & -.02755 & -.75216 & 1.58055 \\ \mathrm{C} & .35762 & -1.13442 & 2.87556 \\ \mathrm{C} & .92728 & -.20182 & 3.74643 \\ \mathrm{H} & 1.54789 & 1.84863 & 4.02056 \\ \mathrm{H} & .86099 & 2.53918 & 1.72776 \\ \mathrm{H} & -.15333 & .88003 & .18499 \\ \mathrm{H} & .20847 & -2.15519 & 3.21642 \\ \mathrm{H} & 1.22085 & -.51114 & 4.74650 \\ \mathrm{C} & -3.04455 & -4.03822 & 4.59206 \\ \mathrm{C} & -3.04893 & -2.64139 & 4.51646 \\ \mathrm{C} & -2.77384 & -2.00111 & 3.30741 \\ \mathrm{C} & -2.48459 & -2.74336 & 2.15349 \\ \mathrm{C} & -2.49291 & -4.14238 & 2.23695 \\ \mathrm{C} & -2.76766 & -4.78846 & 3.44683 \\ \mathrm{H} & -3.26929 & -4.53674 & 5.53224 \\ \mathrm{H} & -3.27848 & -2.04812 & 5.39891 \\ \mathrm{H} & -2.80750 & -.91972 & 3.23605 \\ \mathrm{H} & -2.32245 & -4.74213 & 1.34408 \\ \mathrm{H} & -2.78206 & -5.87514 & 3.48789 \\ & & & \end{array}$

\section{Syn transoid betaine (conformer 4)}

$\mathrm{E}($ B3LYP 6-31G*) $=-1661.853708$ $\mathrm{E}\left(\right.$ B3LYP $\left.6-311+\mathrm{G}^{* *}\right)=$

$-1662.169856$

$\mathrm{E}\left(\mathrm{B} 3 \mathrm{LYP} 6-31 \mathrm{G}^{*}\left(\mathrm{CH}_{3} \mathrm{CN}\right)\right)=$ $-1661.933879$

$\mathrm{E}\left(\mathrm{B} 3 \mathrm{LYP} 6-311+\mathrm{G} * *\left(\mathrm{CH}_{3} \mathrm{CN}\right)\right)=$ $-1662.227368$

$\begin{array}{cccc}\mathrm{C} & -1.57174 & -1.77061 & .19803 \\ \mathrm{~N} & -2.31649 & -.53776 & .00575 \\ \mathrm{H} & -1.58650 & -2.40573 & -.70396 \\ \mathrm{H} & .30425 & -.89628 & -.45126 \\ \mathrm{~S} & -2.16136 & .18237 & -1.39602 \\ \mathrm{O} & -.85058 & .89236 & -1.62902 \\ \mathrm{O} & -2.52551 & -.66344 & -2.58667 \\ \mathrm{C} & -.04601 & -1.40725 & .44961 \\ \mathrm{~S} & 1.02774 & -2.96592 & .53998 \\ \mathrm{C} & .91695 & -3.66573 & -1.14124\end{array}$

H $\quad-.08048 \quad-4.08625 \quad-1.27666$

H $\quad 1.11955 \quad-2.89376-1.88770$

$\begin{array}{llll}\mathrm{H} & 1.65911 & -4.46602 & -1.19944\end{array}$

$\begin{array}{llll}\text { C } & 2.72092 & -2.28556 & .50578\end{array}$

$\begin{array}{llll}\mathrm{H} & 2.83623 & -1.59923 & -.33659\end{array}$

$\begin{array}{llll}\mathrm{H} & 2.88343 & -1.77045 & 1.45360\end{array}$

$\begin{array}{lllll}\mathrm{H} & 3.40784 & -3.13161 & .41850\end{array}$

$\begin{array}{llll}\text { C } & -3.40443 & 1.47774 & -1.27347\end{array}$

$\begin{array}{llll}\mathrm{H} & -3.35232 & 2.06384 & -2.19489\end{array}$

H $\quad-4.39296 \quad 1.02783 \quad-1.16786$

$\begin{array}{llll}\mathrm{H} & -3.18152 & 2.10890 & -.41150\end{array}$

$\begin{array}{llll}\mathrm{C} & .82512 & 1.13653 & 3.83556\end{array}$

$\begin{array}{llll}\text { C } & .80492 & 1.64868 & 2.53523\end{array}$

$\begin{array}{llll}\text { C } & .53369 & .80837 & 1.45453\end{array}$

$\begin{array}{llll}\text { C } & .26711 & -.55422 & 1.66020\end{array}$

$\begin{array}{llll}\text { C } & .28683 & -1.06191 & 2.96939\end{array}$

$\begin{array}{llll}\text { C } & .56875 & -.22045 & 4.04857\end{array}$

$\begin{array}{llll}\mathrm{H} & 1.03884 & 1.79102 & 4.67731\end{array}$

$\begin{array}{llll}\mathrm{H} & .99961 & 2.70426 & 2.35977\end{array}$

$\begin{array}{llll}\mathrm{H} & .49500 & 1.20652 & .44505\end{array}$

$\begin{array}{llll}\mathrm{H} & .07382 & -2.11063 & 3.15701\end{array}$

$\begin{array}{llll}\mathrm{H} & .58072 & -.62690 & 5.05687\end{array}$

$\begin{array}{llll}\text { C } & -3.39704 & -4.06723 & 3.39995\end{array}$

$\begin{array}{llll}\text { C } & -3.39851 & -2.66909 & 3.44547\end{array}$

$\begin{array}{llll}\text { C } & -2.80730 & -1.93049 & 2.41950\end{array}$

$\begin{array}{llll}\text { C } & -2.19931 & -2.57471 & 1.33245\end{array}$

$\begin{array}{llll}\text { C } & -2.21280 & -3.97545 & 1.29056\end{array}$

$\begin{array}{llll}\text { C } & -2.80394 & -4.71982 & 2.31694\end{array}$

H $\quad-3.86736 \quad-4.64159 \quad 4.19480$

$\begin{array}{llll}\mathrm{H} & -3.87127 & -2.15140 & 4.27720\end{array}$

$\begin{array}{llll}\mathrm{H} & -2.83369 & -.84664 & 2.43536\end{array}$

$\begin{array}{llll}\mathrm{H} & -1.79382 & -4.49882 & .43233\end{array}$

$\begin{array}{llll}\mathrm{H} & -2.81534 & -5.80579 & 2.25913\end{array}$

\section{Anti transoid betaine}

$\mathrm{E}\left(\mathrm{B} 3 \mathrm{LYP} 6-31 \mathrm{G}^{*}\right)=$
-1661.846748
$\mathrm{E}\left(\mathrm{B} 3 \mathrm{LYP} 6-311+\mathrm{G}^{* *}\right)=$
-1662.160320
$\mathrm{E}\left(\mathrm{B} 3 \mathrm{LYP} 6-31 \mathrm{G}^{*}\left(\mathrm{CH}_{3} \mathrm{CN}\right)\right)=$
-1661.936676
$\mathrm{E}\left(\mathrm{B} 3 \mathrm{LYP} 6-311+\mathrm{G}^{* *}\left(\mathrm{CH}_{3} \mathrm{CN}\right)\right)=$
-1662.232241

$\begin{array}{llll}\text { C } & -1.96910 & -1.78604 & .56247\end{array}$

$\begin{array}{llll}\mathrm{N} & -2.64525 & -.53687 & .85822\end{array}$

$\begin{array}{llll}\mathrm{H} & -2.30030 & -2.22479 & -.38753\end{array}$

$\begin{array}{llll}\mathrm{H} & -.11096 & -.93393 & 1.33499\end{array}$

$\begin{array}{llll}\mathrm{S} & -3.69340 & .04038 & -.18011\end{array}$

$\begin{array}{llll}\mathrm{O} & -3.88001 & -.79550 & -1.40893\end{array}$

$\begin{array}{llll}\mathrm{O} & -4.97225 & .40552 & .50586\end{array}$

$\begin{array}{llll}\text { C } & -.45318 & -1.41205 & .41290\end{array}$

$\begin{array}{llll}\mathrm{S} & .62513 & -3.00733 & .30586\end{array}$

$\begin{array}{llll}\text { C } & 2.21450 & -2.41566 & -.36617\end{array}$

$\begin{array}{llll}\mathrm{H} & 2.05959 & -2.16779 & -1.41666\end{array}$

$\begin{array}{llll}\mathrm{H} & 2.56841 & -1.54646 & .19310\end{array}$

$\begin{array}{llll}\mathrm{H} & 2.91885 & -3.24727 & -.28023\end{array}$

$\begin{array}{llll}\text { C } & 1.12190 & -3.33593 & 2.03271\end{array}$

H $1.50459 \quad-2.42035 \quad 2.49025$

H $\quad .24954 \quad-3.70693 \quad 2.56942$

$\begin{array}{llll}\mathrm{H} & 1.89698 & -4.10594 & 1.99861\end{array}$

$\begin{array}{llll}\text { C } & -3.07955 & 1.64081 & -.75801\end{array}$
$\begin{array}{llll}\mathrm{H} & -3.85983 & 2.10012 & -1.37127\end{array}$

$\begin{array}{llll}\mathrm{H} & -2.87216 & 2.27218 & .10911\end{array}$

H $\quad-2.17276 \quad 1.49572-1.34445$

$\begin{array}{llll}\text { C } & -2.54565 & -4.77609 & 3.66772\end{array}$

$\begin{array}{llll}\text { C } & -2.38652 & -3.42734 & 4.00929\end{array}$

$\begin{array}{llll}\text { C } & -2.21511 & -2.46591 & 3.01211\end{array}$

$\begin{array}{llll}\text { C } & -2.19344 & -2.83175 & 1.65725\end{array}$

$\begin{array}{llll}\text { C } & -2.36595 & -4.18339 & 1.32564\end{array}$

$\begin{array}{llll}\text { C } & -2.54024 & -5.15126 & 2.32242\end{array}$

$\begin{array}{llll}\mathrm{H} & -2.68450 & -5.52425 & 4.44494\end{array}$

$\begin{array}{llll}\mathrm{H} & -2.40317 & -3.12504 & 5.05419\end{array}$

$\begin{array}{llll}\mathrm{H} & -2.11483 & -1.41858 & 3.27787\end{array}$

$\begin{array}{llll}\mathrm{H} & -2.37826 & -4.48307 & .27927\end{array}$

$\begin{array}{llll}\mathrm{H} & -2.68124 & -6.19356 & 2.04565\end{array}$

$\begin{array}{llll}\text { C } & .31789 & 1.19107 & -2.94297\end{array}$

$\begin{array}{llll}\text { C } & .72744 & 1.55271 & -1.65645\end{array}$

$\begin{array}{llll}\text { C } & .50058 & .68903 & -.58236\end{array}$

$\begin{array}{llll}\text { C } & -.15247 & -.53672 & -.77844\end{array}$

$\begin{array}{lllll}\text { C } & -.54798 & -.89944 & -2.07633\end{array}$

$\begin{array}{llll}\text { C } & -.31117 & -.04099 & -3.15078\end{array}$

$\mathrm{H} \quad \begin{array}{llll}48436 & 1.86568 & -3.77936\end{array}$

$\mathrm{H} \quad 1.213892 .51006 \quad-1.48556$

$\begin{array}{llll}\mathrm{H} & .80658 & .98061 & .41941\end{array}$

$\begin{array}{llll}\mathrm{H} & -1.06049 & -1.84116 & -2.25323\end{array}$

$\begin{array}{llll}\mathrm{H} & -.63345 & -.32859 & -4.14847\end{array}$

\section{Anti transoid betaine (conformer 1)}

\author{
E(B3LYP 6-31G*) $=$ \\ $-1661.830456$ \\ $\mathrm{E}\left(\mathrm{B} 3 \mathrm{LYP} 6-311+\mathrm{G}^{* *}\right)=$ \\ $-1662.160401$ \\ $\mathrm{E}\left(\mathrm{B} 3 \mathrm{LYP} 6-31 \mathrm{G} *\left(\mathrm{CH}_{3} \mathrm{CN}\right)\right)=$ \\ $-1661.936456$ \\ $\mathrm{E}\left(\mathrm{B} 3 \mathrm{LYP}\right.$ 6-311+G**( $\left.\left.\mathrm{CH}_{3} \mathrm{CN}\right)\right)=$ \\ $-1662.231721$
}

$\begin{array}{cccc}\mathrm{C} & -1.94302 & -1.82437 & .58641 \\ \mathrm{~N} & -2.68507 & -.57946 & .64514 \\ \mathrm{H} & -2.22931 & -2.44029 & -.27588 \\ \mathrm{H} & -.14449 & -.75700 & 1.22364 \\ \mathrm{~S} & -3.75916 & -.27034 & -.47769 \\ \mathrm{O} & -3.86643 & -1.31655 & -1.54410 \\ \mathrm{O} & -5.07336 & .11280 & .12793 \\ \mathrm{C} & -.44409 & -1.40225 & .39302 \\ \mathrm{~S} & .71194 & -2.93516 & .57272 \\ \mathrm{C} & 2.28499 & -2.38945 & -.17443 \\ \mathrm{H} & 2.13400 & -2.31972 & -1.25217 \\ \mathrm{H} & 2.59180 & -1.42664 & .24071 \\ \mathrm{H} & 3.02467 & -3.16354 & .04788 \\ \mathrm{C} & 1.18906 & -2.93705 & 2.33640 \\ \mathrm{H} & 1.51699 & -1.93816 & 2.63415 \\ \mathrm{H} & .32415 & -3.25469 & 2.91736 \\ \mathrm{H} & 2.00072 & -3.66129 & 2.44553 \\ \mathrm{C} & -3.24656 & 1.24702 & -1.31751 \\ \mathrm{H} & -4.04625 & 1.54114 & -2.00310 \\ \mathrm{H} & -3.09514 & 2.02927 & -.57021 \\ \mathrm{H} & -2.32306 & 1.06617 & -1.86704 \\ \mathrm{C} & -2.43430 & -4.24615 & 4.16470 \\ \mathrm{C} & -2.35432 & -2.85150 & 4.26353 \\ \mathrm{C} & -2.21126 & -2.07313 & 3.11396 \\ \mathrm{C} & -2.13879 & -2.67080 & 1.84608\end{array}$




$\begin{array}{cccc}\mathrm{C} & -2.23300 & -4.06717 & 1.75725 \\ \mathrm{C} & -2.37874 & -4.85209 & 2.90759 \\ \mathrm{H} & -2.55103 & -4.85224 & 5.06030 \\ \mathrm{H} & -2.41071 & -2.37083 & 5.23779 \\ \mathrm{H} & -2.17334 & -.99108 & 3.19065 \\ \mathrm{H} & -2.20576 & -4.54686 & .78052 \\ \mathrm{H} & -2.45862 & -5.93306 & 2.81818 \\ \mathrm{C} & .25002 & .63348 & -3.34933 \\ \mathrm{C} & .60188 & 1.23612 & -2.13831 \\ \mathrm{C} & .40195 & .55558 & -.93498 \\ \mathrm{C} & -.16660 & -.72646 & -.92691 \\ \mathrm{C} & -.50676 & -1.33004 & -2.14839 \\ \mathrm{C} & -.29552 & -.65457 & -3.35105 \\ \mathrm{H} & .39655 & 1.16414 & -4.28704 \\ \mathrm{H} & 1.02246 & 2.23881 & -2.12725 \\ \mathrm{H} & .66336 & 1.03334 & .00619 \\ \mathrm{H} & -.95623 & -2.31928 & -2.16764 \\ \mathrm{H} & -.57272 & -1.12939 & -4.28900\end{array}$

\section{Anti transoid betaine (conformer 2)}

$\mathrm{E}\left(\mathrm{B} 3 \mathrm{LYP} 6-31 \mathrm{G}^{*}\right)=$
-1661.861736
$\mathrm{E}\left(\mathrm{B} 3 \mathrm{LYP} 6-311+\mathrm{G}^{* *}\right)=$
-1662.160310
$\mathrm{E}\left(\mathrm{B} 3 \mathrm{LYP} 6-31 \mathrm{G}^{*}\left(\mathrm{CH}_{3} \mathrm{CN}\right)\right)=$
-1661.937198
$\mathrm{E}\left(\mathrm{B} 3 \mathrm{LYP} 6-311+\mathrm{G}^{* *}\left(\mathrm{CH}_{3} \mathrm{CN}\right)\right)=$
-1662.232698

$\begin{array}{cccc}\mathrm{C} & -1.88011 & -1.71306 & .542725 \\ \mathrm{~N} & -2.51758 & -.44733 & .856713 \\ \mathrm{H} & -2.22600 & -2.12780 & -.412773 \\ \mathrm{H} & .00471 & -.93486 & 1.326730 \\ \mathrm{~S} & -3.55402 & .16994 & -.170797 \\ \mathrm{O} & -3.77309 & -.65035 & -1.404612 \\ \mathrm{O} & -4.81730 & .56758 & .526055 \\ \mathrm{C} & -.35440 & -1.38427 & .396463 \\ \mathrm{~S} & .66359 & -3.02156 & .268682 \\ \mathrm{C} & 2.25763 & -2.50581 & -.452437 \\ \mathrm{H} & 2.08329 & -2.25804 & -1.500086 \\ \mathrm{H} & 2.66755 & -1.65030 & .089220 \\ \mathrm{H} & 2.92534 & -3.36873 & -.381980 \\ \mathrm{C} & 1.18493 & -3.34753 & 1.988791 \\ \mathrm{H} & 1.62080 & -2.44402 & 2.422286 \\ \mathrm{H} & .30570 & -3.66351 & 2.549688 \\ \mathrm{H} & 1.92024 & -4.15583 & 1.955588 \\ \mathrm{C} & -2.89617 & 1.75620 & -.739874 \\ \mathrm{H} & -3.66323 & 2.23995 & -1.351146 \\ \mathrm{H} & -2.67273 & 2.37713 & .130656 \\ \mathrm{H} & -1.99336 & 1.58991 & -1.326812 \\ \mathrm{C} & -2.57325 & -4.72424 & 3.604979 \\ \mathrm{C} & -2.35895 & -3.38834 & 3.966491 \\ \mathrm{C} & -2.14676 & -2.42011 & 2.983760 \\ \mathrm{C} & -2.13827 & -2.76634 & 1.623408 \\ \mathrm{C} & -2.36412 & -4.10518 & 1.272530 \\ \mathrm{C} & -2.57960 & -5.07964 & 2.254569 \\ \mathrm{H} & -2.74479 & -5.47715 & 4.370965 \\ \mathrm{H} & -2.36530 & -3.10088 & 5.015614 \\ \mathrm{H} & -2.00630 & -1.38160 & 3.265853 \\ \mathrm{H} & -2.38581 & -4.38904 & .221952 \\ \mathrm{H} & -2.76140 & -6.11121 & 1.961965\end{array}$

$\begin{array}{lccl}\mathrm{C} & .51099 & 1.25347 & -2.908387 \\ \mathrm{C} & .93682 & 1.57233 & -1.615996 \\ \mathrm{C} & .67857 & .69607 & -.559313 \\ \mathrm{C} & -.02416 & -.49806 & -.777849 \\ \mathrm{C} & -.43550 & -.81849 & -2.082023 \\ \mathrm{C} & -.16664 & .05161 & -3.139351 \\ \mathrm{H} & .70234 & 1.93783 & -3.731431 \\ \mathrm{H} & 1.46139 & 2.50581 & -1.426846 \\ \mathrm{H} & .99917 & .95459 & .446919 \\ \mathrm{H} & -.98711 & -1.73420 & -2.276314 \\ \mathrm{H} & -.50155 & -.20230 & -4.141997\end{array}$

Anti transoid betaine (conformer 3)

E(B3LYP 6-31G*) $=$
-1661.831601
E(B3LYP 6-311+G**)=
-1662.154924
E(B3LYP 6-31G*( $\left.\left(\mathrm{CH}_{3} \mathrm{CN}\right)\right)=$
-1661.932521
$\mathrm{E}\left(\mathrm{B} 3 \mathrm{LYP} 6-311+\mathrm{G}^{* *}\left(\mathrm{CH}_{3} \mathrm{CN}\right)\right)=$
-1662.229990

$\begin{array}{llll}\text { C } & -1.68425 & -1.69798 & .10426\end{array}$

$\begin{array}{llll}\mathrm{N} & -2.59381 & -.55807 & .09755\end{array}$

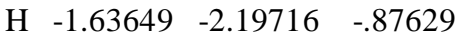

$\mathrm{H} \quad-.28350 \quad-.66283 \quad 1.41451$

$\begin{array}{llll}\text { S } & -4.05801 & -.79994 & -.45897\end{array}$

$\begin{array}{llll}\mathrm{O} & -4.64816 & .52099 & -.82042\end{array}$

$\begin{array}{llll}\text { O } & -4.14891 & -1.84923 & -1.52288\end{array}$

$\begin{array}{llll}\text { C } & -.28826 & -1.08313 & .40560\end{array}$

$\begin{array}{llll}\mathrm{S} & .93012 & -2.58424 & .57672\end{array}$

$\begin{array}{llll}\text { C } & 2.52733 & -2.02818 & -.09978\end{array}$

H $\quad 2.41215 \quad-1.91860-1.17916$

$\begin{array}{llll}\mathrm{H} & 2.83974 & -1.08734 & .35706\end{array}$

$\begin{array}{llll}\mathrm{H} & 3.24344 & -2.82593 & .11694\end{array}$

$\begin{array}{llll}\text { C } & 1.30465 & -2.58492 & 2.36275\end{array}$

$\begin{array}{llll}\mathrm{H} & 1.68001 & -1.60483 & 2.66796\end{array}$

H $\quad .37788 \quad-2.82601 \quad 2.88608$

$\begin{array}{llll}\mathrm{H} & 2.05180 & -3.36240 & 2.54105\end{array}$

$\begin{array}{llll}\text { C } & -5.18056 & -1.39126 & .84286\end{array}$

$\begin{array}{llll}\mathrm{H} & -6.18384 & -1.44529 & .41067\end{array}$

$\begin{array}{llll}\mathrm{H} & -4.86683 & -2.37667 & 1.18934\end{array}$

$\begin{array}{llll}\mathrm{H} & -5.17072 & -.67864 & 1.67045\end{array}$

$\begin{array}{llll}\text { C } & -3.13882 & -4.65283 & 2.93485\end{array}$

$\begin{array}{llll}\text { C } & -2.78604 & -3.37247 & 3.37894\end{array}$

$\begin{array}{llll}\text { C } & -2.27254 & -2.43663 & 2.48106\end{array}$

$\begin{array}{llll}\text { C } & -2.08975 & -2.76566 & 1.12699\end{array}$

$\begin{array}{llll}\text { C } & -2.43365 & -4.05360 & .69755\end{array}$

$\begin{array}{llll}\text { C } & -2.95917 & -4.99177 & 1.59198\end{array}$

$\begin{array}{llll}\mathrm{H} & -3.56168 & -5.37534 & 3.62887\end{array}$

$\begin{array}{llll}\mathrm{H} & -2.93372 & -3.09532 & 4.42018\end{array}$

$\begin{array}{llll}\mathrm{H} & -2.05779 & -1.42761 & 2.82483\end{array}$

$\begin{array}{llll}\mathrm{H} & -2.31491 & -4.31085 & -.35300\end{array}$

$\begin{array}{llll}\mathrm{H} & -3.24073 & -5.97974 & 1.23481\end{array}$

$\begin{array}{llll}\text { C } & 1.45786 & 1.78009 & -2.31036\end{array}$

$\begin{array}{llll}\text { C } & 1.56918 & 1.94861 & -.92810\end{array}$

$\begin{array}{llll}\text { C } & .97941 & 1.02382 & -.06333\end{array}$

$\begin{array}{llll}\text { C } & .26639 & -.07648 & -.56613\end{array}$

$\begin{array}{llll}\text { C } & .15539 & -.23528 & -1.95671\end{array}$

$\begin{array}{llll}\text { C } & .74725 & .68777 & -2.82050\end{array}$

$\begin{array}{llll}\mathrm{H} & 1.91675 & 2.49778 & -2.98639\end{array}$
$\begin{array}{llll}\mathrm{H} & 2.11044 & 2.79936 & -.52095\end{array}$

$\begin{array}{llll}\mathrm{H} & 1.05933 & 1.16804 & 1.01258\end{array}$

$\begin{array}{llll}\mathrm{H} & -.39329 & -1.07665 & -2.37145\end{array}$

$\begin{array}{llll}\mathrm{H} & .65049 & .55589 & -3.89544\end{array}$

\section{Syn elimination TS}

$\mathrm{E}\left(\mathrm{B} 3 \mathrm{LYP} 6-31 \mathrm{G}^{*}\right)=-1661.884855$

$\mathrm{E}\left(\right.$ B3LYP $\left.6-311+\mathrm{G}^{* *}\right)=$

$-1662.170064$

E $\left(\right.$ B3LYP 6-31G* $\left.*\left(\mathrm{CH}_{3} \mathrm{CN}\right)\right)=$ $-1661.924136$

$\mathrm{E}\left(\mathrm{B} 3 \mathrm{LYP} 6-311+\mathrm{G}^{* *}\left(\mathrm{CH}_{3} \mathrm{CN}\right)\right)=$ $-1662.216357$

$$
\begin{array}{cccc}
\mathrm{C} & -1.53387 & -1.59997 & .40679 \\
\mathrm{~N} & -1.95250 & -.20243 & .46079 \\
\mathrm{H} & -1.67726 & -2.06250 & -.57954 \\
\mathrm{H} & .35662 & -.95337 & -.37872 \\
\mathrm{~S} & -2.15424 & .53999 & -.95009 \\
\mathrm{O} & -.90391 & 1.13045 & -1.52456 \\
\mathrm{O} & -2.91713 & -.27232 & -1.95137 \\
\mathrm{C} & -.04536 & -1.32319 & .56324 \\
\mathrm{~S} & 1.08700 & -3.38228 & .23860 \\
\mathrm{C} & 2.74774 & -2.78731 & -.22415 \\
\mathrm{H} & 2.68705 & -2.40468 & -1.24618 \\
\mathrm{H} & 3.07399 & -1.99491 & .45595 \\
\mathrm{H} & 3.44983 & -3.62521 & -.19190 \\
\mathrm{C} & 1.41320 & -4.03656 & 1.90885 \\
\mathrm{H} & 1.77819 & -3.24374 & 2.56692 \\
\mathrm{H} & .47119 & -4.43387 & 2.29327 \\
\mathrm{H} & 2.14956 & -4.84242 & 1.83827 \\
\mathrm{C} & -3.19405 & 1.92815 & -.47608 \\
\mathrm{H} & -3.36702 & 2.52344 & -1.37691 \\
\mathrm{H} & -4.13952 & 1.55454 & -.07890 \\
\mathrm{H} & -2.67281 & 2.52402 & .27495 \\
\mathrm{C} & 1.96969 & .49988 & 3.87445 \\
\mathrm{C} & 2.32641 & .80170 & 2.55670 \\
\mathrm{C} & 1.65928 & .18739 & 1.49853 \\
\mathrm{C} & .61654 & -.73333 & 1.73501 \\
\mathrm{C} & .27643 & -1.03724 & 3.06902 \\
\mathrm{C} & .94618 & -.42108 & 4.12457 \\
\mathrm{H} & 2.48424 & .98054 & 4.70278 \\
\mathrm{H} & 3.11624 & 1.52017 & 2.35276 \\
\mathrm{H} & 1.91873 & .44804 & .47523 \\
\mathrm{H} & -.51577 & -1.74531 & 3.28206 \\
\mathrm{H} & .66597 & -.65803 & 5.14780 \\
\mathrm{C} & -3.73552 & -4.08739 & 3.19951 \\
\mathrm{C} & -3.74816 & -2.69649 & 3.33853 \\
\mathrm{C} & -3.02385 & -1.89327 & 2.45399 \\
\mathrm{C} & -2.27257 & -2.46720 & 1.42072 \\
\mathrm{C} & -2.27790 & -3.86299 & 1.27864 \\
\mathrm{C} & -2.99765 & -4.66990 & 2.16455 \\
\mathrm{H} & -4.30821 & -4.71135 & 3.88175 \\
\mathrm{H} & -4.33274 & -2.23200 & 4.12968 \\
\mathrm{H} & -3.05203 & -.81204 & 2.53896 \\
\mathrm{H} & -1.74657 & -4.32393 & .44768 \\
\mathrm{H} & -2.99945 & -5.74943 & 2.03220 \\
& & & \\
& &
\end{array}
$$

\section{Syn elimination TS (conformer)}

$\mathrm{E}\left(\mathrm{B} 3 \mathrm{LYP} 6-31 \mathrm{G}^{*}\right)=-1661.878084$ 


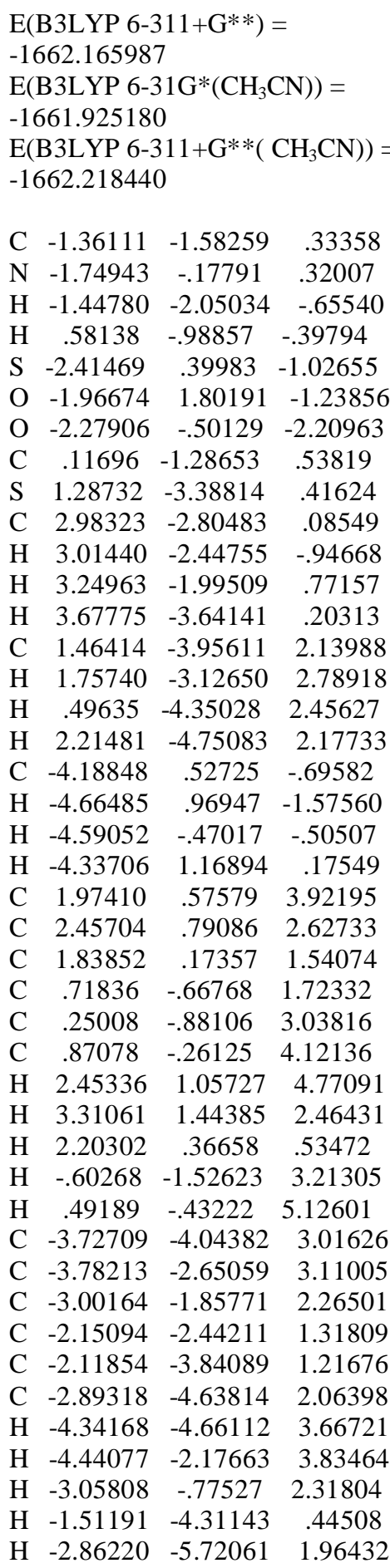

\section{Anti elimination TS}

$\mathrm{E}\left(\mathrm{B} 3 \mathrm{LYP} 6-31 \mathrm{G}^{*}\right)=-1661.882439$

$\mathrm{E}\left(\right.$ B3LYP $\left.6-311+\mathrm{G}^{* *}\right)=$

$-1662.168318$

$\mathrm{E}\left(\mathrm{B} 3 \mathrm{LYP} 6-31 \mathrm{G}^{*}\left(\mathrm{CH}_{3} \mathrm{CN}\right)\right)=$ $-1661.928329$

$\mathrm{E}\left(\mathrm{B} 3 \mathrm{LYP} 6-311+\mathrm{G}^{* *}\left(\mathrm{CH}_{3} \mathrm{CN}\right)\right)=$ $-1662.221239$

$\begin{array}{llll}\text { C } & -1.87314 & -1.75339 & .50355\end{array}$ $\begin{array}{llll}\mathrm{N} & -2.42929 & -.44007 & .85937\end{array}$

$\begin{array}{llll}\text { H } & -2.25378 & -2.13592 & -.44875 \\ \mathrm{H} & -.04147 & -.92611 & 1.32468 \\ \mathrm{~S} & -3.35848 & .31557 & -.22621 \\ \mathrm{O} & -3.43596 & -.37550 & -1.54628 \\ \mathrm{O} & -4.67912 & .63684 & .38606 \\ \mathrm{C} & -. .45700 & -1.24896 & .37505 \\ \mathrm{~S} & .98849 & -3.19552 & .43127 \\ \mathrm{C} & .69467 & -3.98188 & -1.18706 \\ \mathrm{H} & -.28616 & -4.46212 & -1.14687 \\ \mathrm{H} & .71186 & -3.23357 & -1.98466 \\ \mathrm{H} & 1.46159 & -4.74129 & -1.36353 \\ \mathrm{C} & 2.63079 & -2.45261 & .15386 \\ \mathrm{H} & 2.59525 & -1.73521 & -.67037 \\ \mathrm{H} & 2.91972 & -1.94079 & 1.07563 \\ \mathrm{H} & 3.35353 & -3.24441 & -.06255 \\ \mathrm{C} & -2.59583 & 1.92371 & -.51166 \\ \mathrm{H} & -3.25709 & 2.48776 & -1.17534 \\ \mathrm{H} & -2.49916 & 2.44224 & .44467 \\ \mathrm{H} & -1.61751 & 1.78650 & -.97341 \\ \mathrm{C} & -2.62320 & -4.68725 & 3.60531 \\ \mathrm{C} & -2.14189 & -3.41634 & 3.94117 \\ \mathrm{C} & -1.89006 & -2.47577 & 2.94239 \\ \mathrm{C} & -2.11153 & -2.78814 & 1.59244 \\ \mathrm{C} & -2.59431 & -4.06111 & 1.26576 \\ \mathrm{C} & -2.84916 & -5.00731 & 2.26509 \\ \mathrm{H} & -2.82807 & -5.41710 & 4.38523 \\ \mathrm{H} & -1.97308 & -3.15467 & 4.98350 \\ \mathrm{H} & -1.54571 & -1.48150 & 3.21500 \\ \mathrm{H} & -2.79603 & -4.31138 & .22556 \\ \mathrm{H} & -3.23412 & -5.98795 & 1.99484 \\ \mathrm{C} & .99319 & 1.03054 & -2.94090 \\ \mathrm{C} & 1.44106 & 1.29399 & -1.64148 \\ \mathrm{C} & .97356 & .52669 & -.57633 \\ \mathrm{C} & .03561 & -.50782 & -.78722 \\ \mathrm{C} & -. .39959 & -.76744 & -2.10423 \\ \mathrm{C} & .07949 & -.00427 & -3.16769 \\ \mathrm{H} & 1.35224 & 1.63137 & -3.77285 \\ \mathrm{H} & 2.14655 & 2.10053 & -1.45895 \\ \mathrm{H} & 1.30713 & .74438 & .43577 \\ \mathrm{H} & -1.13284 & -1.54196 & -2.29686 \\ \mathrm{H} & -.27154 & -.21025 & -4.17546 \\ & & & \end{array}$

\section{Cis aziridine}

$\mathrm{E}\left(\mathrm{B} 3 \mathrm{LYP} 6-31 \mathrm{G}^{*}\right)=$

$-1183.915447$

$\mathrm{E}\left(\mathrm{B} 3 \mathrm{LYP} 6-311+\mathrm{G}^{* *}\right)=$

$-1184.156401$

$\mathrm{E}\left(\mathrm{B} 3 \mathrm{LYP} 6-31 \mathrm{G} *\left(\mathrm{CH}_{3} \mathrm{CN}\right)\right)=$

$-1183.949026$

$\mathrm{E}\left(\mathrm{B} 3 \mathrm{LYP} 6-311+\mathrm{G}^{* *}\left(\mathrm{CH}_{3} \mathrm{CN}\right)\right)=$ $-1184.183779$

$\begin{array}{lccc}\mathrm{C} & -1.366931 & -1.09803 & -.16583 \\ \mathrm{C} & .133387 & -.88054 & -.18906 \\ \mathrm{~N} & -.799494 & .26438 & -.17892 \\ \mathrm{H} & -1.720712 & -1.46057 & .80017 \\ \mathrm{H} & .600856 & -1.11610 & .76709 \\ \mathrm{~S} & -.937287 & 1.20460 & 1.23562 \\ \mathrm{O} & -2.293977 & 1.77465 & 1.22724 \\ \mathrm{O} & -.517605 & .44144 & 2.42848 \\ \mathrm{C} & 2.761302 & -1.41494 & -3.55016 \\ \mathrm{C} & 2.823367 & -2.27411 & -2.44794\end{array}$ $\begin{array}{llll}\text { C } & 1.959469 & -2.08950 & -1.36716\end{array}$

C $\quad 1.020901-1.04676-1.37836$

$\begin{array}{llll}\text { C } & .962359 & -.18986 & -2.48496\end{array}$

$\begin{array}{llll}\text { C } & 1.828837 & -.37402 & -3.56483\end{array}$

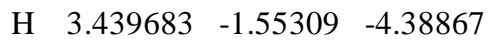

H $3.549852 \quad-3.08293 \quad-2.42628$

$\begin{array}{lllll}\mathrm{H} & 2.016965 & -2.75221 & -.50552\end{array}$

$\begin{array}{llll}\mathrm{H} & .248602 & .62753 & -2.49049\end{array}$

$\begin{array}{llll}\mathrm{H} & 1.778468 & .30264 & -4.41473\end{array}$

$\begin{array}{llll}\text { C } & -3.743827 & -2.45055 & -3.48826\end{array}$

$\begin{array}{llll}\text { C } & -3.339880 & -1.11422 & -3.43101\end{array}$

$\begin{array}{llll}\text { C } & -2.566778 & -.65712 & -2.36007\end{array}$

$\begin{array}{llll}\text { C } & -2.190821 & -1.53373 & -1.33539\end{array}$

C $-2.605157-2.87362 \quad-1.39319$

C $-3.374622 \quad-3.32995 \quad-2.46455$

$\begin{array}{llll}\mathrm{H} & -4.346177 & -2.80509 & -4.32147\end{array}$

$\begin{array}{llll}\mathrm{H} & -3.630340 & -.42206 & -4.21804\end{array}$

$\begin{array}{llll}\mathrm{H} & -2.265795 & .38447 & -2.30835\end{array}$

$\begin{array}{llll}\mathrm{H} & -2.326065 & -3.55930 & -.59528\end{array}$

$\begin{array}{llll}\mathrm{H} & -3.688918 & -4.37052 & -2.49905\end{array}$

$\begin{array}{llll}\text { C } & .262738 & 2.49590 & .91276\end{array}$

$\begin{array}{llll}\mathrm{H} & .230491 & 3.17473 & 1.76991\end{array}$

$\begin{array}{llll}\mathrm{H} & 1.253810 & 2.04505 & .82381\end{array}$

$\begin{array}{llll}\mathrm{H} & -.020035 & 3.01750 & -.00387\end{array}$

\section{Cis aziridine (conformer)}

\begin{tabular}{|c|c|c|c|}
\hline & $\begin{array}{l}4.147019 \\
3 \text { LYP 6-3 }\end{array}$ & $\begin{array}{l}\left.1 \mathrm{G}^{*}\right)= \\
11+\mathrm{G}^{* *} \\
1 \mathrm{G}^{*}(\mathrm{CH} \\
11+\mathrm{G}^{* *}\end{array}$ & $\mathrm{CN}))=$ \\
\hline & & -1.13686 & \\
\hline & .15265 & -.99808 & 18 \\
\hline & -.71926 & .17464 & \\
\hline & -1.73934 & & \\
\hline & .62128 & -1.34413 & .740 \\
\hline & -.79103 & & \\
\hline & .413 & & \\
\hline & -2.11432 & 1.82592 & 1.22 \\
\hline & 2.6596 & & \\
\hline & & & \\
\hline & 1.8 & & \\
\hline & & & \\
\hline & & & \\
\hline & 1.84 & -.449 & -3.55 \\
\hline & 3.29 & & \\
\hline & & & \\
\hline & 1.8 & -2.99 & \\
\hline & .409 & .65623 & -2.38 \\
\hline & & & \\
\hline & & -2.1 & \\
\hline & -3.2 & -.91427 & -3.48 \\
\hline & -2.4 & & \\
\hline & & & \\
\hline & -2.8 & -2.711 & -1.38 \\
\hline & -3.6 & -3.072 & 0 \\
\hline & & & \\
\hline & -3.38050 & -.20678 & -4.298 \\
\hline
\end{tabular}




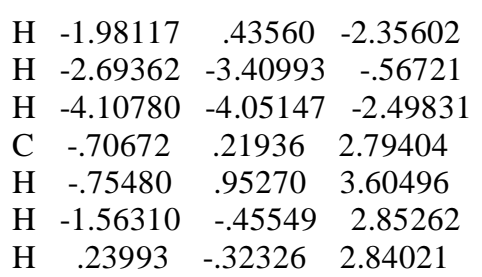

\section{Trans aziridine}

$\mathrm{E}\left(\mathrm{B} 3 \mathrm{LYP} 6-31 \mathrm{G}^{*}\right)=$

$-1183.905585$

$\mathrm{E}\left(\mathrm{B} 3 \mathrm{LYP} 6-311+\mathrm{G}^{* *}\right)=$

$-1184.151084$

$\mathrm{E}\left(\mathrm{B} 3 \mathrm{LYP} 6-31 \mathrm{G}^{*}\left(\mathrm{CH}_{3} \mathrm{CN}\right)\right)=$

$-1183.944300$

$\mathrm{E}\left(\mathrm{B} 3 \mathrm{LYP} 6-311+\mathrm{G} * *\left(\mathrm{CH}_{3} \mathrm{CN}\right)\right)=$ $-1184.178017$

$\begin{array}{cccc}\mathrm{C} & -1.54133 & -.87423 & .12202 \\ \mathrm{C} & -.05571 & -.94715 & -.05250 \\ \mathrm{~N} & -.71317 & .37276 & .12138 \\ \mathrm{H} & .48831 & -1.30362 & .82114 \\ \mathrm{~S} & -. .41867 & 1.28018 & 1.53231 \\ \mathrm{O} & -1.66325 & 2.00859 & 1.82818 \\ \mathrm{O} & .19731 & .47793 & 2.60677 \\ \mathrm{C} & 1.91205 & -1.72613 & -3.79838 \\ \mathrm{C} & 2.28189 & -2.42298 & -2.64481 \\ \mathrm{C} & 1.64065 & -2.15977 & -1.43132 \\ \mathrm{C} & .61944 & -1.20290 & -1.36163 \\ \mathrm{C} & .25382 & -.50457 & -2.52213 \\ \mathrm{C} & .89700 & -.76522 & -3.73282 \\ \mathrm{H} & 2.41484 & -1.92483 & -4.74180 \\ \mathrm{H} & 3.07404 & -3.16666 & -2.68594 \\ \mathrm{H} & 1.93626 & -2.69847 & -.53289 \\ \mathrm{H} & -.52035 & .25637 & -2.46963 \\ \mathrm{H} & .60989 & -.21299 & -4.62462 \\ \mathrm{H} & -2.13284 & -.89714 & -.79367 \\ \mathrm{C} & .80867 & 2.43228 & .90823 \\ \mathrm{H} & 1.07349 & 3.08996 & 1.74120 \\ \mathrm{H} & 1.68451 & 1.86931 & .57668 \\ \mathrm{H} & .37201 & 3.00158 & .08527 \\ \mathrm{C} & -3.80226 & -2.49305 & 3.41325 \\ \mathrm{C} & -4.34302 & -1.49735 & 2.59285 \\ \mathrm{C} & -3.58701 & -.96703 & 1.54719 \\ \mathrm{C} & -2.27749 & -1.41736 & 1.31249 \\ \mathrm{C} & -1.74371 & -2.41635 & 2.13681 \\ \mathrm{C} & -2.50272 & -2.95095 & 3.18171 \\ \mathrm{H} & -4.39344 & -2.91373 & 4.22327 \\ \mathrm{H} & -5.35563 & -1.13937 & 2.76286 \\ \mathrm{H} & -4.01595 & -.20317 & .90233 \\ \mathrm{H} & -.74245 & -2.79741 & 1.95689 \\ \mathrm{H} & -2.07945 & -3.73211 & 3.80852\end{array}$

\section{Trans aziridine (conformer)}

$\mathrm{E}\left(\mathrm{B} 3 \mathrm{LYP} 6-31 \mathrm{G}^{*}\right)=-1183.769517$

$\mathrm{E}\left(\mathrm{B} 3 \mathrm{LYP} 6-311+\mathrm{G}^{* *}\right)=$

$-1184.142579$

$\mathrm{E}\left(\mathrm{B} 3 \mathrm{LYP} 6-31 \mathrm{G}^{*}\left(\mathrm{CH}_{3} \mathrm{CN}\right)\right)=$

$-1183.937750$

$\begin{array}{lccc}\mathrm{E}(\mathrm{B} 3 \mathrm{LYP} & \left.6-311+\mathrm{G}^{* *}\left(\mathrm{CH}_{3} \mathrm{CN}\right)\right)= \\ -1184.173497 & & \\ \mathrm{H} & & & \\ \mathrm{C} & -1.56269 & -.93051 & .10265 \\ \mathrm{C} & -.07339 & -1.06674 & -.06769 \\ \mathrm{~N} & -.67512 & .25863 & .18456 \\ \mathrm{H} & .45100 & -1.52055 & .77308 \\ \mathrm{~S} & -.34416 & 1.25324 & 1.51258 \\ \mathrm{O} & .94043 & 1.89105 & 1.18660 \\ \mathrm{O} & -1.54111 & 2.09089 & 1.68057 \\ \mathrm{C} & 1.92537 & -1.66014 & -3.83661 \\ \mathrm{C} & 2.45262 & -2.19883 & -2.66063 \\ \mathrm{C} & 1.79281 & -2.00345 & -1.44372 \\ \mathrm{C} & .60191 & -1.26798 & -1.38874 \\ \mathrm{C} & .08110 & -.72362 & -2.57345 \\ \mathrm{C} & .73727 & -.92199 & -3.78884 \\ \mathrm{H} & 2.43750 & -1.80880 & -4.78436 \\ \mathrm{H} & 3.38021 & -2.76545 & -2.68659 \\ \mathrm{H} & 2.21158 & -2.41837 & -.52918 \\ \mathrm{H} & -.82860 & -.12844 & -2.54891 \\ \mathrm{H} & .32362 & -.49607 & -4.69983 \\ \mathrm{H} & -2.15072 & -.88283 & -.81445 \\ \mathrm{C} & -.09685 & .33337 & 3.03977 \\ \mathrm{H} & .19721 & 1.08795 & 3.77603 \\ \mathrm{H} & -1.02886 & -.14563 & 3.33704 \\ \mathrm{H} & .71025 & -.39061 & 2.91364 \\ \mathrm{C} & -3.85172 & -2.31258 & 3.47283 \\ \mathrm{C} & -4.32911 & -1.28462 & 2.65241 \\ \mathrm{C} & -3.57452 & -.85598 & 1.56075 \\ \mathrm{C} & -2.33189 & -1.44761 & 1.27937 \\ \mathrm{C} & -1.86934 & -2.49102 & 2.09094 \\ \mathrm{C} & -2.62492 & -2.91758 & 3.18732 \\ \mathrm{H} & -4.43713 & -2.64330 & 4.32716 \\ \mathrm{H} & -5.28777 & -.81661 & 2.86239 \\ \mathrm{H} & -3.94671 & -.05767 & .92243 \\ \mathrm{H} & -.92156 & -2.97480 & 1.87023 \\ \mathrm{H} & -2.25321 & -3.72239 & 3.81648\end{array}$

\section{Stabilized ylide} reaction:

\section{$\mathrm{Me}_{2} \mathrm{SCHCO}_{2} \mathrm{Me}+$} $\mathrm{PhCH}=\mathrm{NSO}_{2} \mathrm{Me}$

\section{$\mathrm{Me}_{2} \mathrm{SCHCO}_{2} \mathrm{Me}$}

$\mathrm{E}\left(\mathrm{B} 3 \mathrm{LYP} 6-31 \mathrm{G}^{*}\right)=-745.117050$

$\mathrm{E}\left(\right.$ B3LYP $\left.6-311+\mathrm{G}^{* *}\right)=$

$-745.263793$

$\mathrm{E}\left(\mathrm{B} 3 \mathrm{LYP} 6-31 \mathrm{G}^{*}\left(\mathrm{CH}_{3} \mathrm{CN}\right)\right)=$

$-745.151019$

$\mathrm{E}\left(\mathrm{B} 3 \mathrm{LYP} 6-311+\mathrm{G}^{* *}\left(\mathrm{CH}_{3} \mathrm{CN}\right)\right)=$

$-745.291809$

$\mathrm{E}(\mathrm{B} 3 \mathrm{LYP} 6-311+\mathrm{G} * *(\mathrm{THF}))=$ $-745.287492$

$\begin{array}{llll}\text { C } & 1.30506 & -.06226 & 0.00000\end{array}$

$\begin{array}{llll}\mathrm{H} & 2.24433 & -.60509 & 0.00000\end{array}$

$\begin{array}{llll}\mathrm{S} & -2.03521 & .24258 & .034175\end{array}$

$\begin{array}{llll}\text { C } & -2.46146 & .36313 & -1.745482\end{array}$

$\begin{array}{llll}\text { C } & -.92866 & -1.03878 & .216541\end{array}$ $\begin{array}{llll}\text { C } & .26494 & -.77534 & .933131\end{array}$

$\begin{array}{llll}\text { O } & .63610 & .29142 & 1.444921\end{array}$

$\begin{array}{llll}\text { O } & 1.05931 & -1.90231 & 1.024728\end{array}$

$\begin{array}{llll}\text { C } & 2.28821 & -1.73197 & 1.744064\end{array}$

$\begin{array}{llll}\text { C } & -3.66261 & -.31144 & .676238\end{array}$

$\begin{array}{llll}\mathrm{H} & -3.25418 & 1.10821 & -1.864458\end{array}$

$\begin{array}{llll}\mathrm{H} & -2.78714 & -.61008 & -2.123395\end{array}$

$\begin{array}{llll}\mathrm{H} & -1.55732 & .69073 & -2.263950\end{array}$

$\begin{array}{llll}\mathrm{H} & -1.17147 & -2.02081 & -.172854\end{array}$

H $\quad 2.77571 \quad-2.70950 \quad 1.731916$

$\begin{array}{llll}\mathrm{H} & 2.93298 & -.98958 & 1.263438\end{array}$

$\begin{array}{llll}\mathrm{H} & 2.10675 & -1.42381 & 2.778336\end{array}$

$\begin{array}{llll}\mathrm{H} & -3.55605 & -.42059 & 1.758033\end{array}$

$\begin{array}{llll}\mathrm{H} & -3.93935 & -1.26619 & .220762\end{array}$

$\begin{array}{llll}\mathrm{H} & -4.40862 & .45621 & .449166\end{array}$

\section{$\mathrm{Me}_{2} \mathrm{SCHCO}_{2} \mathrm{Me}$ (conformer 1)}

$\mathrm{E}\left(\mathrm{B} 3 \mathrm{LYP} 6-31 \mathrm{G}^{*}\right)=-745.130234$

$\mathrm{E}\left(\mathrm{B} 3 \mathrm{LYP} 6-311+\mathrm{G}^{* *}\right)=$

$-745.270354$

$\mathrm{E}\left(\mathrm{B} 3 \mathrm{LYP} 6-31 \mathrm{G} *\left(\mathrm{CH}_{3} \mathrm{CN}\right)\right)=$ $-745.149819$

$\mathrm{E}\left(\mathrm{B} 3 \mathrm{LYP} 6-311+\mathrm{G}^{* *}\left(\mathrm{CH}_{3} \mathrm{CN}\right)\right)=$ $-745.288274$

$\begin{array}{llll}\text { S } & -2.10894 & .18565 & .09093\end{array}$

$\begin{array}{llll}\text { C } & -2.52286 & .90836 & 1.72754\end{array}$

$\begin{array}{llll}\text { C } & -.90801 & -1.00715 & .29488\end{array}$

$\begin{array}{llll}\text { C } & .30928 & -.74607 & .96510\end{array}$

$\begin{array}{llll}\mathrm{O} & .67025 & .32890 & 1.47174\end{array}$

$\begin{array}{llll}\mathrm{O} & 1.11211 & -1.87100 & 1.00587\end{array}$

$\begin{array}{llll}\text { C } & 2.38592 & -1.76703 & 1.65939\end{array}$

$\begin{array}{llll}\text { C } & -1.35556 & 1.66257 & -.69811\end{array}$

$\begin{array}{llll}\text { H } & -3.20929 & 1.74542 & 1.57156\end{array}$

$\begin{array}{llll}\mathrm{H} & -1.60244 & 1.23139 & 2.21564\end{array}$

$\begin{array}{llll}\mathrm{H} & -3.01429 & .12231 & 2.30563\end{array}$

$\begin{array}{llll}\mathrm{H} & -1.15637 & -1.97588 & -.11691\end{array}$

$\begin{array}{llll}\mathrm{H} & 2.42192 & -2.50533 & 2.46678\end{array}$

$\begin{array}{llll}\mathrm{H} & 3.17150 & -1.99163 & .93047\end{array}$

$\begin{array}{llll}\mathrm{H} & 2.52793 & -.76466 & 2.06312\end{array}$

$\begin{array}{lllll}\mathrm{H} & -1.09094 & 1.37382 & -1.71787\end{array}$

$\begin{array}{llll}\mathrm{H} & -.46846 & 1.95239 & -.13352\end{array}$

$\begin{array}{llll}\mathrm{H} & -2.09965 & 2.46387 & -.71527\end{array}$

\section{$\mathrm{Me}_{2} \mathrm{SCHCO}_{2} \mathrm{Me}$ (conformer 2)}

$\mathrm{E}\left(\mathrm{B} 3 \mathrm{LYP} 6-31 \mathrm{G}^{*}\right)=-745.113974$

$\mathrm{E}\left(\mathrm{B} 3 \mathrm{LYP} 6-311+\mathrm{G}^{* *}\right)=$

$-745.262972$

$\mathrm{E}\left(\mathrm{B} 3 \mathrm{LYP} 6-31 \mathrm{G}^{*}\left(\mathrm{CH}_{3} \mathrm{CN}\right)\right)=$

$-745.148114$

$\mathrm{E}\left(\mathrm{B} 3 \mathrm{LYP} 6-311+\mathrm{G} * *\left(\mathrm{CH}_{3} \mathrm{CN}\right)\right)=$ $-745.288905$

$\begin{array}{llll}\text { S } & -2.16119 & .20616 & .16886\end{array}$

$\begin{array}{llll}\text { C } & -2.40561 & .91325 & 1.84455\end{array}$

$\begin{array}{llll}\text { C } & -.98517 & -1.03746 & .26685\end{array}$

$\begin{array}{llll}\text { C } & .27607 & -.79634 & .84784\end{array}$

$\begin{array}{llll}\mathrm{O} & .67614 & .28739 & 1.31170\end{array}$

O $\quad \begin{array}{llll}1.18911 & -1.83006 \quad .93115\end{array}$

$\begin{array}{llll}\text { C } & .85904 & -3.13734 & .45388\end{array}$ 
$\begin{array}{llll}\text { C } & -1.41688 & 1.66004 & -.66716\end{array}$

H $\quad-3.07000 \quad 1.77811 \quad 1.76239$

H $-1.43340 \quad 1.19163 \quad 2.25213$

$\begin{array}{llll}\mathrm{H} & -2.87352 & .13357 & 2.45025\end{array}$

H $-1.32098-1.98325-.13056$

H $\quad \begin{array}{llll}1.73253 & -3.75991 & .66274\end{array}$

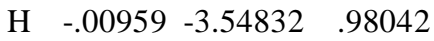

H $\quad .66740 \quad-3.13228 \quad-.62529$

H $\quad-1.24894 \quad 1.37596-1.70866$

H $\quad-.47636 \quad 1.90656 \quad-.17345$

H $\quad-2.12938 \quad 2.48806 \quad-.61289$

\section{Syn cisoid addition TS}

$\mathrm{E}\left(\mathrm{B} 3 \mathrm{LYP} 6-31 \mathrm{G}^{*}\right)=-1658.704864$

$\mathrm{E}\left(\mathrm{B} 3 \mathrm{LYP} 6-311+\mathrm{G}^{* *}\right)=$

$-1659.006645$

$\mathrm{E}\left(\mathrm{B} 3 \mathrm{LYP} 6-31 \mathrm{G}^{*}\left(\mathrm{CH}_{3} \mathrm{CN}\right)\right)=$ $-1658.744495$

$\mathrm{E}\left(\mathrm{B} 3 \mathrm{LYP} 6-311+\mathrm{G} * *\left(\mathrm{CH}_{3} \mathrm{CN}\right)\right)=$ $-1659.049392$

$\mathrm{E}\left(\mathrm{B} 3 \mathrm{LYP} 6-311+\mathrm{G}^{* *}(\mathrm{THF})\right)=$ $-1659.041684$

$\begin{array}{lccc}\mathrm{C} & -1.86236 & -1.76220 & .31022 \\ \mathrm{~N} & -2.09841 & -2.62187 & -.70398 \\ \mathrm{H} & -1.80787 & -2.15180 & 1.32868 \\ \mathrm{H} & .05407 & -.49462 & 1.32414 \\ \mathrm{~S} & -2.04534 & -4.22524 & -.41290 \\ \mathrm{O} & -1.33636 & -4.90188 & -1.52421 \\ \mathrm{O} & -1.59831 & -4.57150 & .96483 \\ \mathrm{C} & .14557 & -1.17119 & .47830 \\ \mathrm{~S} & .58566 & -.22035 & -.95716 \\ \mathrm{C} & 2.40633 & -.03179 & -.96674 \\ \mathrm{H} & 2.67281 & .60063 & -.11675 \\ \mathrm{H} & 2.87305 & -1.01490 & -.89212 \\ \mathrm{H} & 2.67656 & .46488 & -1.90317 \\ \mathrm{C} & .34886 & -1.32145 & -2.39473 \\ \mathrm{H} & 1.08290 & -2.12506 & -2.36051 \\ \mathrm{H} & -.65944 & -1.73210 & -2.31709 \\ \mathrm{H} & .45998 & -.70016 & -3.28664 \\ \mathrm{C} & -3.77382 & -4.72864 & -.54609 \\ \mathrm{H} & -3.81030 & -5.81782 & -.44817 \\ \mathrm{H} & -4.34650 & -4.25429 & .25424 \\ \mathrm{H} & -4.15545 & -4.42229 & -1.52263 \\ \mathrm{C} & -3.92815 & 2.00627 & .03879 \\ \mathrm{C} & -3.79431 & 1.19950 & -1.09539 \\ \mathrm{C} & -3.10897 & -.01348 & -1.01655 \\ \mathrm{C} & -2.53662 & -.43104 & .19498 \\ \mathrm{C} & -2.67462 & .38429 & 1.32845 \\ \mathrm{C} & -3.36852 & 1.59284 & 1.25267 \\ \mathrm{H} & -4.47407 & 2.94480 & -.01980 \\ \mathrm{H} & -4.23744 & 1.50605 & -2.04031 \\ \mathrm{H} & -3.04088 & -.66157 & -1.88425 \\ \mathrm{H} & -2.25497 & .05935 & 2.27873 \\ \mathrm{H} & -3.48228 & 2.20674 & 2.14280 \\ \mathrm{C} & .90787 & -2.39681 & .73174 \\ \mathrm{O} & .81979 & -2.76232 & 2.03002 \\ \mathrm{O} & 1.49734 & -3.06586 & -.11101 \\ \mathrm{C} & 1.35052 & -4.06441 & 2.35743 \\ \mathrm{H} & 1.21424 & -4.17167 & 3.43451 \\ \mathrm{H} & .78863 & -4.83236 & 1.82422 \\ \mathrm{H} & 2.41081 & -4.12682 & 2.10097\end{array}$

\section{Syn cisoid addition TS (conformer 1)}

$\mathrm{E}\left(\mathrm{B} 3 \mathrm{LYP} 6-31 \mathrm{G}^{*}\right)=-1658.696238$
$\mathrm{E}\left(\mathrm{B} 3 \mathrm{LYP} 6-311+\mathrm{G}^{* *}\right)=$
-1658.993215
$\mathrm{E}\left(\mathrm{B} 3 \mathrm{LYP} 6-31 \mathrm{G}^{*}\left(\mathrm{CH}_{3} \mathrm{CN}\right)\right)=$
-1658.739484
$\mathrm{E}\left(\mathrm{B} 3 \mathrm{LYP} 6-311+\mathrm{G}^{* *}\left(\mathrm{CH}_{3} \mathrm{CN}\right)\right)=$
-1659.046294

$\begin{array}{llll}\text { C } & -1.63140 & -1.44378 & .43901\end{array}$

$\begin{array}{llll}\mathrm{N} & -1.90997 & -2.76624 & .41105\end{array}$

H $\quad-1.64554 \quad-.92608 \quad 1.40055$

$\begin{array}{llll}\mathrm{H} & .54094 & .00479 & .52725\end{array}$

$\begin{array}{llll}\mathrm{S} & -2.16449 & -3.55588 & 1.81161\end{array}$

$\begin{array}{llll}\mathrm{O} & -1.25599 & -4.72514 & 1.94068\end{array}$

$\begin{array}{llll}\mathrm{O} & -2.23143 & -2.66451 & 3.00201\end{array}$

$\begin{array}{llll}\text { C } & .42768 & -1.03566 & .22895\end{array}$

S $\quad \begin{array}{llll}\text { 9 } & 91709 & -1.33811 & -1.47435\end{array}$

$\begin{array}{llll}\mathrm{C} & .69525 & .28906 & -2.26566\end{array}$

$\mathrm{H} \quad-.37080 \quad .50054 \quad-2.32312$

H $\quad 1.21837 \quad 1.05765 \quad-1.69120$

$\begin{array}{lllll}\mathrm{H} & 1.11364 & .20587 & -3.27200\end{array}$

C $\quad 2.74966-1.42406 \quad-1.51764$

H $3.17554 \quad-.54517 \quad-1.02778$

H $3.03469-2.34079 \quad-1.00280$

H $3.04174 \quad-1.47029 \quad-2.57079$

C $\quad-3.81443 \quad-4.24331 \quad 1.56492$

H $\quad-4.04816 \quad-4.85921 \quad 2.43828$

$\begin{array}{llll}\mathrm{H} & -4.53318 & -3.42624 & 1.47017\end{array}$

$\begin{array}{llll}\mathrm{H} & -3.81348 & -4.85554 & .66052\end{array}$

$\begin{array}{llll}\text { C } & -3.35308 & .84861 & -2.76959\end{array}$

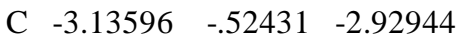

C $-2.56962-1.26930 \quad-1.89422$

$\begin{array}{llll}\text { C } & -2.19562 & -.64752 & -.69217\end{array}$

$\begin{array}{lllll}\text { C } & -2.42158 & .72848 & -.53578\end{array}$

$\begin{array}{llll}\text { C } & -3.00015 & 1.47166 & -1.56714\end{array}$

H $\quad-3.80536 \quad 1.42656 \quad-3.57187$

H $-3.41993 \quad-1.01857 \quad-3.85566$

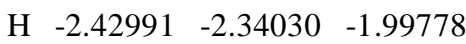

$\begin{array}{llll}\mathrm{H} & -2.15408 & 1.21374 & .40105\end{array}$

H $\quad-3.18200 \quad 2.53481 \quad-1.43053$

C $\quad 1.02138-2.04592 \quad 1.10107$

$\begin{array}{llll}\text { O } & .95754 & -1.70130 & 2.40167\end{array}$

$\begin{array}{llll}\text { O } & 1.45315 & -3.12784 & .71108\end{array}$

C $1.39133 \quad-2.70951 \quad 3.34337$

H $\quad 1.26334 \quad-2.25987 \quad 4.32849$

$\mathrm{H} \quad \begin{array}{llll}77215 & -3.60226 & 3.24341\end{array}$

H $\quad 2.44139 \quad-2.96241 \quad 3.17438$

\section{Syn cisoid addition TS (conformer 2)}

$\mathrm{E}\left(\mathrm{B} 3 \mathrm{LYP} 6-31 \mathrm{G}^{*}\right)=-1658.705211$

$\mathrm{E}\left(\mathrm{B} 3 \mathrm{LYP} 6-311+\mathrm{G}^{* *}\right)=$

$-1659.005794$

$\mathrm{E}\left(\mathrm{B} 3 \mathrm{LYP} 6-31 \mathrm{G}^{*}\left(\mathrm{CH}_{3} \mathrm{CN}\right)\right)=$

$-1658.744333$

$\mathrm{E}\left(\mathrm{B} 3 \mathrm{LYP} 6-311+\mathrm{G}^{* *}\left(\mathrm{CH}_{3} \mathrm{CN}\right)\right)=$ $-1659.049244$ $\begin{array}{llll}\text { C } & -1.67549 & -1.45825 & .47185\end{array}$

$\begin{array}{llll}\mathrm{N} & -1.79922 & -2.77867 & .21966\end{array}$

H $\quad-1.77840 \quad-1.10486 \quad 1.49971$

$\begin{array}{llll}\mathrm{H} & .14847 & .26278 & .73178\end{array}$

S $-1.91762-3.81531 \quad 1.47400$

$\begin{array}{llll}\mathrm{O} & -1.19099 & -5.06067 & 1.13355\end{array}$

O $\quad-1.60552 \quad-3.20185 \quad 2.79352$

$\begin{array}{llll}\text { C } & .32647 & -.78408 & .49949\end{array}$

$\begin{array}{lllll}\mathrm{S} & .99106 & -.88987 & -1.14500\end{array}$

$\begin{array}{llll}\text { C } & 2.80252 & -.64705 & -1.03195\end{array}$

$\begin{array}{llll}\mathrm{H} & 2.97371 & .39115 & -.73783\end{array}$

H $3.21506 \quad-1.34133 \quad-.29859$

H $3.22073 \quad-.82795 \quad-2.02659$

C $\quad .91104 \quad-2.65189-1.62011$

H $\quad 1.61807 \quad-3.22197 \quad-1.01949$

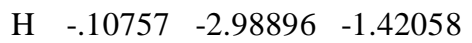

H $\quad 1.14535 \quad-2.70151 \quad-2.68621$

C $\quad-3.67015 \quad-4.25149 \quad 1.50376$

H $\quad-3.80891 \quad-5.01193 \quad 2.27811$

H $\quad-4.25707 \quad-3.35976 \quad 1.73589$

$\begin{array}{llll}\mathrm{H} & -3.95119 & -4.64960 & .52620\end{array}$

$\begin{array}{llll}\text { C } & -3.49848 & 1.16176 & -2.41718\end{array}$

$\begin{array}{llll}\text { C } & -3.25302 & -.17551 & -2.74386\end{array}$

C $\quad-2.64452 \quad-1.02398 \quad-1.81804$

$\begin{array}{llll}\text { C } & -2.25965 & -.54263 & -.55680\end{array}$

$\begin{array}{llll}\text { C } & -2.50943 & .80064 & -.23637\end{array}$

$\begin{array}{llll}\text { C } & -3.12783 & 1.64650 & -1.15807\end{array}$

$\mathrm{H} \quad-3.98575 \quad 1.81864 \quad-3.13372$

$\mathrm{H} \quad-3.55026 \quad-.56448 \quad-3.71519$

$\begin{array}{llll}\mathrm{H} & -2.49270 & -2.07273 & -2.05311\end{array}$

$\begin{array}{llll}\mathrm{H} & -2.23779 & 1.17800 & .74776\end{array}$

$\begin{array}{llll}\mathrm{H} & -3.33122 & 2.68048 & -.89023\end{array}$

$\begin{array}{llll}\text { C } & .99670 & -1.55908 & 1.54578\end{array}$

$\begin{array}{llll}\text { O } & .73331 & -1.05320 & 2.77092\end{array}$

$\begin{array}{llll}\mathrm{O} & 1.65820 & -2.57849 & 1.37380\end{array}$

C $\quad 1.16884 \quad-1.85341 \quad 3.89089$

$\begin{array}{llll}\mathrm{H} & .88584 & -1.28851 & 4.78020\end{array}$

H $\quad .65758 \quad-2.81652 \quad 3.87295$

H $\quad 2.25131 \quad-2.00075 \quad 3.86400$

\section{Syn transoid addition TS}

$\mathrm{E}\left(\mathrm{B} 3 \mathrm{LYP} 6-31 \mathrm{G}^{*}\right)=-1658.704769$

$\mathrm{E}\left(\mathrm{B} 3 \mathrm{LYP} 6-311+\mathrm{G}^{* *}\right)=$

$-1659.006135$

$\mathrm{E}\left(\mathrm{B} 3 \mathrm{LYP} 6-31 \mathrm{G} *\left(\mathrm{CH}_{3} \mathrm{CN}\right)\right)=$

$-1658.745082$

$\mathrm{E}\left(\mathrm{B} 3 \mathrm{LYP} 6-311+\mathrm{G} * *\left(\mathrm{CH}_{3} \mathrm{CN}\right)\right)=$ $-1659.050395$

$\mathrm{E}\left(\mathrm{B} 3 \mathrm{LYP} 6-311+\mathrm{G}^{* *}(\mathrm{THF})\right)=$ $-1659.042304$

$\begin{array}{lccl}\mathrm{C} & -1.71748 & -1.63107 & .348036 \\ \mathrm{~N} & -2.37579 & -.48926 & .037483 \\ \mathrm{H} & -1.60029 & -2.39810 & -.422748 \\ \mathrm{H} & .34974 & -.27881 & -.166875 \\ \mathrm{~S} & -2.34949 & -.02976 & -1.519704 \\ \mathrm{O} & -1.22680 & .91191 & -1.801229 \\ \mathrm{O} & -2.43268 & -1.17667 & -2.473806 \\ \mathrm{C} & .33711 & -1.18438 & .435362 \\ \mathrm{~S} & 1.24211 & -2.53752 & -.307198 \\ \mathrm{C} & .93019 & -2.27341 & -2.081871 \\ \mathrm{H} & -.14165 & -2.37123 & -2.264693\end{array}$




\begin{tabular}{|c|c|c|c|}
\hline \multicolumn{4}{|c|}{ 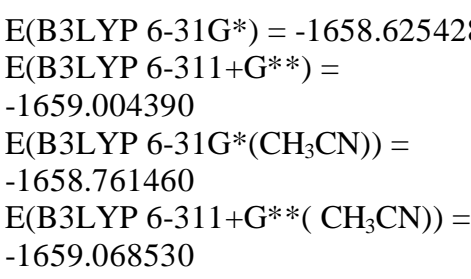 } \\
\hline & & & \\
\hline & & & \\
\hline & & & \\
\hline & & & \\
\hline & & & \\
\hline & & & \\
\hline & & & \\
\hline & & & \\
\hline & & & \\
\hline & & & \\
\hline & -.2 & & \\
\hline & & & \\
\hline & & & \\
\hline & & & \\
\hline & & & \\
\hline & & & \\
\hline & & & \\
\hline & & & \\
\hline & & & \\
\hline & & & \\
\hline & & & \\
\hline & & & \\
\hline & & & \\
\hline & & & \\
\hline & & & \\
\hline & & & \\
\hline & & & \\
\hline & & & \\
\hline & & 1.70 & -1.1 \\
\hline & & & \\
\hline & & & \\
\hline & -3.73 & & \\
\hline & & & \\
\hline & & & \\
\hline & & & \\
\hline & 1.10810 & 1.39968 & 2.08709 \\
\hline & -0 & 50000 & \\
\hline & & & \\
\hline & & & \\
\hline
\end{tabular}

Syn cisoid betaine (conformer 1)

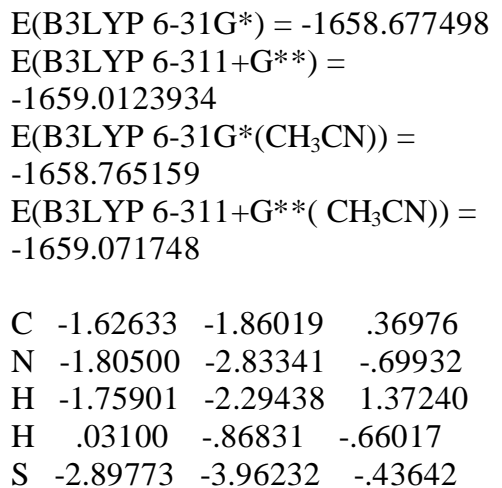

$\begin{array}{cccc}\mathrm{O} & -2.63924 & -4.75998 & .81528 \\ \mathrm{O} & -4.32440 & -3.52228 & -.52801 \\ \mathrm{C} & -.13080 & -1.42327 & .26791 \\ \mathrm{~S} & .87288 & -2.97151 & .00552 \\ \mathrm{C} & 2.57078 & -2.42973 & .41604 \\ \mathrm{H} & 2.86060 & -1.65736 & -.30119 \\ \mathrm{H} & 2.63686 & -2.06582 & 1.44386 \\ \mathrm{H} & 3.20721 & -3.30887 & .28474 \\ \mathrm{C} & .51144 & -4.03435 & 1.44214 \\ \mathrm{H} & .64717 & -3.46915 & 2.36494 \\ \mathrm{H} & -.51547 & -4.39663 & 1.33400 \\ \mathrm{H} & 1.21697 & -4.86741 & 1.37756 \\ \mathrm{C} & -2.62683 & -5.07798 & -1.82074 \\ \mathrm{H} & -3.34423 & -5.89600 & -1.71162 \\ \mathrm{H} & -2.80740 & -4.54820 & -2.75779 \\ \mathrm{H} & -1.60595 & -5.46261 & -1.78726 \\ \mathrm{C} & -4.03868 & 1.74912 & .15417 \\ \mathrm{C} & -3.72223 & 1.04352 & -1.01146 \\ \mathrm{C} & -2.95930 & -.12356 & -.94144 \\ \mathrm{C} & -2.50391 & -.60349 & .29269 \\ \mathrm{C} & -2.83415 & .10206 & 1.45813 \\ \mathrm{C} & -3.59459 & 1.27339 & 1.39047 \\ \mathrm{H} & -4.63530 & 2.65697 & .09943 \\ \mathrm{H} & -4.07570 & 1.39951 & -1.97677 \\ \mathrm{H} & -2.73003 & -.68548 & -1.84143 \\ \mathrm{H} & -2.50727 & -.27119 & 2.42711 \\ \mathrm{H} & -3.84752 & 1.80702 & 2.30383 \\ \mathrm{C} & .34032 & -.60567 & 1.46320 \\ \mathrm{O} & .66311 & .63606 & 1.10342 \\ \mathrm{O} & .39052 & -1.03163 & 2.60260 \\ \mathrm{C} & 1.04045 & 1.54221 & 2.17228 \\ \mathrm{H} & 1.24849 & 2.49192 & 1.68004 \\ \mathrm{H} & .21659 & 1.64710 & 2.88178 \\ \mathrm{H} & 1.92853 & 1.16618 & 2.68512 \\ & & & \end{array}$

Syn cisoid betaine (conformer 2)

$\mathrm{E}\left(\mathrm{B} 3 \mathrm{LYP} 6-31 \mathrm{G}^{*}\right)=-1658.627601$
$\mathrm{E}\left(\mathrm{B} 3 \mathrm{LYP} 6-311+\mathrm{G}^{* *}\right)=$
-1659.008834
$\mathrm{E}\left(\mathrm{B} 3 \mathrm{LYP} 6-31 \mathrm{G}^{*}\left(\mathrm{CH}_{3} \mathrm{CN}\right)\right)=$
-1658.759964
$\mathrm{E}\left(\mathrm{B} 3 \mathrm{LYP} 6-311+\mathrm{G}^{* * *}\left(\mathrm{CH}_{3} \mathrm{CN}\right)\right)=$
-1659.066188

$\begin{array}{llll}\text { C } & -1.83098 & -1.64552 & .16963\end{array}$

$\begin{array}{llll}\mathrm{N} & -2.23683 & -2.23852 & -1.09589\end{array}$

$\begin{array}{llll}\mathrm{H} & -1.92080 & -2.33581 & 1.01864\end{array}$

$\begin{array}{llll}\mathrm{H} & -.10178 & -.25558 & .48379\end{array}$

$\begin{array}{llll}\mathrm{S} & -2.27417 & -3.83114 & -1.09168\end{array}$

$\begin{array}{lllll}\mathrm{O} & -1.04751 & -4.45130 & -.48768\end{array}$

$\begin{array}{lllll}\mathrm{O} & -3.53238 & -4.43426 & -.54371\end{array}$

$\begin{array}{llll}\text { C } & -.28380 & -1.27399 & .12580\end{array}$

S $\quad .28114 \quad-1.34536 \quad-1.64049$

$\begin{array}{llll}\text { C } & -.66089 & .00336 & -2.43099\end{array}$

$\begin{array}{llll}\mathrm{H} & -1.69343 & -.33669 & -2.49230\end{array}$

$\begin{array}{llll}\mathrm{H} & -.56833 & .92734 & -1.85635\end{array}$

$\begin{array}{llll}\mathrm{H} & -.23154 & .12640 & -3.42912\end{array}$

$\begin{array}{llll}\text { C } & 1.92098 & -.53303 & -1.55441\end{array}$

$\begin{array}{llll}\mathrm{H} & 1.86066 & .41771 & -1.01869\end{array}$

$\mathrm{H} \quad 2.60344 \quad-1.22393 \quad-1.06070$

$\begin{array}{llll}\mathrm{H} & 2.23504 & -.37054 & -2.58927\end{array}$

$\begin{array}{llll}\text { C } & -2.28267 & -4.20926 & -2.84985\end{array}$
$\begin{array}{llll}\mathrm{H} & -2.37273 & -5.29444 & -2.94970\end{array}$

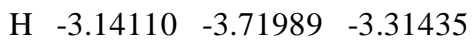

$\begin{array}{llll}\mathrm{H} & -1.35280 & -3.86197 & -3.30342\end{array}$

$\begin{array}{llll}\text { C } & -4.26776 & 1.77071 & 1.30760\end{array}$

$\begin{array}{llll}\text { C } & -4.59235 & 1.02579 & .17178\end{array}$

$\begin{array}{llll}\text { C } & -3.80011 & -.06262 & -.20942\end{array}$

$\begin{array}{llll}\text { C } & -2.67170 & -.41744 & .53545\end{array}$

$\begin{array}{llll}\text { C } & -2.35827 & .32994 & 1.68263\end{array}$

$\begin{array}{llll}\text { C } & -3.14610 & 1.41649 & 2.06525\end{array}$

$\begin{array}{llll}\mathrm{H} & -4.88502 & 2.61495 & 1.60618\end{array}$

$\begin{array}{llll}\mathrm{H} & -5.46863 & 1.28562 & -.41840\end{array}$

H $-4.05392 \quad-.65936-1.07919$

$\begin{array}{llll}\mathrm{H} & -1.50321 & .05066 & 2.29572\end{array}$

$\begin{array}{llll}\mathrm{H} & -2.88973 & 1.98135 & 2.95874\end{array}$

$\begin{array}{llll}\text { C } & .60703 & -2.21692 & .92820\end{array}$

$\begin{array}{llll}\text { O } & .20429 & -2.25795 & 2.19886\end{array}$

$\begin{array}{llll}\mathrm{O} & 1.57185 & -2.81150 & .48760\end{array}$

$\begin{array}{llll}\text { C } & .97000 & -3.09847 & 3.09982\end{array}$

$\mathrm{H} \quad \begin{array}{llll}49855 & -2.97641 & 4.07480\end{array}$

$\mathrm{H} \quad \begin{array}{llll}91410 & -4.13868 & 2.77264\end{array}$

$\begin{array}{llll}\mathrm{H} & 2.01067 & -2.76778 & 3.12893\end{array}$

\section{Syn cisoid betaine (conformer 3)}

$\mathrm{E}(\mathrm{B} 3 \mathrm{LYP}$ 6-31G*) $=-1658.646070$

$\mathrm{E}\left(\right.$ B3LYP $\left.6-311+\mathrm{G}^{* *}\right)=$

$-1659.012613$

$\mathrm{E}\left(\mathrm{B} 3 \mathrm{LYP} 6-31 \mathrm{G}^{*}\left(\mathrm{CH}_{3} \mathrm{CN}\right)\right)=$

$-1658.758945$

$\mathrm{E}\left(\mathrm{B} 3 \mathrm{LYP} 6-311+\mathrm{G} * *\left(\mathrm{CH}_{3} \mathrm{CN}\right)\right)=$ $-1659.063944$

$\begin{array}{lll}\text { C }-1.82283 & -1.65255 & .20714\end{array}$

$\begin{array}{llll}\mathrm{N} & -2.26028 & -2.49351 & -.89558\end{array}$

H $-1.88719-2.15455 \quad 1.18248$

$\begin{array}{llll}\mathrm{H} & -.07718 & -.32126 & .50984\end{array}$

$\begin{array}{llll}\text { S } & -2.20409 & -4.05836 & -.60944\end{array}$

$\begin{array}{llll}\text { O } & -.82081 & -4.64796 & -.62314\end{array}$

$\begin{array}{llll}\text { O } & -2.98583 & -4.49350 & .59438\end{array}$

$\begin{array}{llll}\text { C } & -.30721 & -1.28319 & .03358\end{array}$

$\begin{array}{llll}\text { S } & -.06459 & -.91457 & -1.77507\end{array}$

$\begin{array}{llll}\text { C } & 1.51347 & .00798 & -1.76349\end{array}$

$\begin{array}{llll}\mathrm{H} & 1.35649 & .92839 & -1.19566\end{array}$

$\begin{array}{llll}\text { H } & 2.30271 & -.59989 & -1.32048\end{array}$

$\begin{array}{llll}\mathrm{H} & 1.74135 & .25491 & -2.80387\end{array}$

C $\quad .42943 \quad-2.42987 \quad-2.67268$

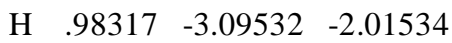

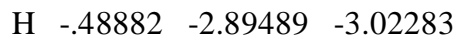

$\begin{array}{llll}\mathrm{H} & 1.04437 & -2.09168 & -3.51113\end{array}$

$\begin{array}{llll}\text { C } & -3.06393 & -4.72648 & -2.04284\end{array}$

H $-3.05227 \quad-5.81552-1.94650$

H $-4.09171 \quad-4.35808-2.04736$

$\begin{array}{llll}\mathrm{H} & -2.55075 & -4.42587 & -2.95883\end{array}$

$\begin{array}{llll}\text { C } & -4.28942 & 1.91094 & .60093\end{array}$

$\begin{array}{llll}\text { C } & -4.56341 & .97400 & -.39787\end{array}$

$\begin{array}{llll}\text { C } & -3.76377 & -.16522 & -.53483\end{array}$

$\begin{array}{lll}\text { C }-2.67823 & -.38335 & .31851\end{array}$

$\begin{array}{lll}\text { C }-2.41527 & .55897 & 1.32547\end{array}$

$\begin{array}{lll}\text { C }-3.21141 & 1.69704 & 1.46692\end{array}$

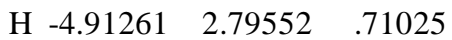

H -5.40551 $1.12408-1.07013$

H $-3.98356 \quad-.91385-1.28741$

$\begin{array}{llll}\text { H } & -1.59528 & .39694 & 2.02390\end{array}$ 


\begin{abstract}
H $-2.99495 \quad 2.41186 \quad 2.25755$
$\begin{array}{llll}\text { C } & .70955 & -2.27190 & .61172\end{array}$

$\begin{array}{llll}\text { O } & .32438 & -2.66493 & 1.82293\end{array}$

$\begin{array}{llll}\text { O } & 1.76518 & -2.57759 & .09076\end{array}$

$\begin{array}{llll}\text { C } & 1.21762 & -3.56333 & 2.52872\end{array}$

H $\quad .75455 \quad-3.72011 \quad 3.50293$

H $1.29589 \quad-4.50511 \quad 1.98178$

H $\quad 2.20412 \quad-3.10678 \quad 2.63620$
\end{abstract}

\section{Anti cisoid betaine}

$\mathrm{E}\left(\mathrm{B} 3 \mathrm{LYP} 6-31 \mathrm{G}^{*}\right)=-1658.669381$

$\mathrm{E}($ B3LYP 6-311+G**) $=$

$-1659.007421$

$\mathrm{E}\left(\mathrm{B} 3 \mathrm{LYP} 6-31 \mathrm{G}^{*}\left(\mathrm{CH}_{3} \mathrm{CN}\right)\right)=$

$-1658.759294$

$\mathrm{E}\left(\mathrm{B} 3 \mathrm{LYP} 6-311+\mathrm{G}^{* *}\left(\mathrm{CH}_{3} \mathrm{CN}\right)\right)=$ $-1659.066478$

$\begin{array}{lccl}\mathrm{C} & -1.70711 & -1.61426 & .424293 \\ \mathrm{~N} & -2.35949 & -.32145 & .516917 \\ \mathrm{H} & -1.91535 & -2.12204 & -.528190 \\ \mathrm{H} & .08233 & -1.00304 & -.684413 \\ \mathrm{~S} & -3.85664 & -.21186 & .003537 \\ \mathrm{O} & -4.14515 & 1.22616 & -.260009 \\ \mathrm{O} & -4.17186 & -1.14413 & -1.124427 \\ \mathrm{C} & -.16629 & -1.29071 & .340191 \\ \mathrm{~S} & .19157 & .21138 & 1.399136 \\ \mathrm{C} & 2.01692 & .24571 & 1.541229 \\ \mathrm{H} & 2.31672 & -.50841 & 2.266447 \\ \mathrm{H} & 2.48770 & .07429 & .570070 \\ \mathrm{H} & 2.26133 & 1.24549 & 1.911078 \\ \mathrm{C} & -.00156 & 1.58467 & .208709 \\ \mathrm{H} & .62832 & 1.40760 & -.667075 \\ \mathrm{H} & -1.05833 & 1.61554 & -.052127 \\ \mathrm{H} & .30787 & 2.49187 & .734804 \\ \mathrm{C} & -5.03009 & -.67057 & 1.307670 \\ \mathrm{H} & -6.04093 & -.51637 & .919818 \\ \mathrm{H} & -4.88435 & -1.71907 & 1.573671 \\ \mathrm{H} & -4.86124 & -.03524 & 2.179279 \\ \mathrm{C} & -2.91080 & -4.41001 & 3.524502 \\ \mathrm{C} & -2.66320 & -3.07200 & 3.850530 \\ \mathrm{C} & -2.25453 & -2.17285 & 2.864604 \\ \mathrm{C} & -2.08160 & -2.59629 & 1.538935 \\ \mathrm{C} & -2.32959 & -3.93903 & 1.222888 \\ \mathrm{C} & -2.74213 & -4.84225 & 2.207023 \\ \mathrm{H} & -3.24456 & -5.10671 & 4.289974 \\ \mathrm{H} & -2.80261 & -2.72544 & 4.872181 \\ \mathrm{H} & -2.10291 & -1.12607 & 3.113092 \\ \mathrm{H} & -2.22199 & -4.27525 & .193391 \\ \mathrm{H} & -2.94634 & -5.87630 & 1.938896 \\ \mathrm{C} & .67991 & -2.47286 & .787555 \\ \mathrm{O} & .72291 & -3.39112 & -.178498 \\ \mathrm{O} & 1.18807 & -2.59140 & 1.885092 \\ \mathrm{C} & 1.37652 & -4.64604 & .149295 \\ \mathrm{H} & 1.32479 & -5.24193 & -.761421 \\ \mathrm{H} & 2.41502 & -4.46603 & .435136 \\ \mathrm{H} & .84270 & -5.13809 & .965075\end{array}$

Anti cisoid betaine (conformer 1)

\author{
$\mathrm{E}(\mathrm{B} 3 \mathrm{LYP}$ 6-31G*) $=-1658.671417$ \\ $\mathrm{E}\left(\right.$ B3LYP $\left.6-311+\mathrm{G}^{* *}\right)=$ \\ $-1659.002791$ \\ $\mathrm{E}\left(\mathrm{B} 3 \mathrm{LYP} 6-31 \mathrm{G}^{*}\left(\mathrm{CH}_{3} \mathrm{CN}\right)\right)=$ \\ $-1658.756803$ \\ $\mathrm{E}\left(\mathrm{B} 3 \mathrm{LYP} 6-311+\mathrm{G} * *\left(\mathrm{CH}_{3} \mathrm{CN}\right)\right)=$ \\ $-1659.064235$
}

$\begin{array}{cccc}\mathrm{C} & -1.73269 & -1.59985 & .46712 \\ \mathrm{~N} & -2.27110 & -.30331 & .84345 \\ \mathrm{H} & -2.05765 & -1.89806 & -.53860 \\ \mathrm{H} & .06795 & -1.30827 & -.76481 \\ \mathrm{~S} & -3.77594 & .00877 & .44582 \\ \mathrm{O} & -3.97727 & 1.48344 & .52053 \\ \mathrm{O} & -4.20257 & -.62033 & -.84322 \\ \mathrm{C} & -.16256 & -1.37355 & .30307 \\ \mathrm{~S} & .29357 & .33934 & .85761 \\ \mathrm{C} & .11398 & .44003 & 2.67243 \\ \mathrm{H} & -.94981 & .57821 & 2.85745 \\ \mathrm{H} & .50329 & -.46528 & 3.13475 \\ \mathrm{H} & .67875 & 1.32350 & 2.98390 \\ \mathrm{C} & 2.11421 & .30145 & .69142 \\ \mathrm{H} & 2.55810 & -.45894 & 1.33606 \\ \mathrm{H} & 2.34554 & .11520 & -.36106 \\ \mathrm{H} & 2.46447 & 1.29878 & .97028 \\ \mathrm{C} & -4.93952 & -.65647 & 1.66670 \\ \mathrm{H} & -5.94614 & -.35306 & 1.36595 \\ \mathrm{H} & -4.86510 & -1.74478 & 1.68728 \\ \mathrm{H} & -4.70054 & -.24400 & 2.64883 \\ \mathrm{C} & -2.98344 & -4.86047 & 3.06059 \\ \mathrm{C} & -2.69378 & -3.60672 & 3.60735 \\ \mathrm{C} & -2.26160 & -2.56332 & 2.78630 \\ \mathrm{C} & -2.10968 & -2.75367 & 1.40693 \\ \mathrm{C} & -2.40258 & -4.01594 & .86934 \\ \mathrm{C} & -2.83407 & -5.06438 & 1.68638 \\ \mathrm{H} & -3.33718 & -5.66713 & 3.69840 \\ \mathrm{H} & -2.81871 & -3.43734 & 4.67435 \\ \mathrm{H} & -2.07768 & -1.58008 & 3.20905 \\ \mathrm{H} & -2.30597 & -4.17482 & -.20254 \\ \mathrm{H} & -3.07029 & -6.03104 & 1.24717 \\ \mathrm{C} & .67839 & -2.48404 & .92149 \\ \mathrm{O} & .69556 & -3.54401 & .11011 \\ \mathrm{O} & 1.22539 & -2.44879 & 2.00693 \\ \mathrm{C} & 1.30967 & -4.74888 & .63701 \\ \mathrm{H} & 1.24206 & -5.48141 & -.16703 \\ \mathrm{H} & 2.35146 & -4.55918 & .90303 \\ \mathrm{H} & .75527 & -5.08653 & 1.51589\end{array}$

Anti cisoid betaine (conformer 2)

$\mathrm{E}($ B3LYP 6-31G*) $=-1658.687105$

$\mathrm{E}\left(\right.$ B3LYP $\left.6-311+\mathrm{G}^{* *}\right)=$

$-1658.651139$

$\mathrm{E}\left(\mathrm{B} 3 \mathrm{LYP} 6-31 \mathrm{G}^{*}\left(\mathrm{CH}_{3} \mathrm{CN}\right)\right)=$

$-1658.756177$

$\mathrm{E}\left(\mathrm{B} 3 \mathrm{LYP} 6-311+\mathrm{G}^{* *}\left(\mathrm{CH}_{3} \mathrm{CN}\right)\right)=$ $-1658.753367$

$\begin{array}{llll}\text { C } & -1.72207 & -1.53906 & .56333\end{array}$

$\begin{array}{llll}\mathrm{N} & -2.30836 & -.36910 & 1.19359\end{array}$

H $\quad-2.05365-1.64818-.48282$

$\begin{array}{llll}\mathrm{H} & .34415 & -2.10985 & .06068\end{array}$

$\begin{array}{llll}\mathrm{S} & -3.84072 & -.08130 & .88056\end{array}$

\begin{abstract}
$\begin{array}{llll}\text { O } & -4.23673 & -.38516 & -.53638\end{array}$
$\begin{array}{llll}\text { O } & -4.81459 & -.64277 & 1.87177\end{array}$

$\begin{array}{llll}\text { C } & -.19009 & -1.24796 & .48019\end{array}$

$\begin{array}{llll}\text { S } & .03019 & .05633 & -.83290\end{array}$

$\begin{array}{llll}\mathrm{C} & -.18004 & 1.70870 & -.07411\end{array}$

$\begin{array}{llll}\mathrm{H} & -1.00803 & 1.60160 & .62688\end{array}$

$\begin{array}{llll}\mathrm{H} & .73467 & 2.01236 & .43151\end{array}$

$\begin{array}{llll}\mathrm{H} & -.43517 & 2.38021 & -.89775\end{array}$

$\begin{array}{llll}\text { C } & 1.81405 & .01962 & -1.20032\end{array}$

$\begin{array}{llll}\mathrm{H} & 2.38461 & .19188 & -.28667\end{array}$

$\begin{array}{llll}\mathrm{H} & 2.04227 & -.95403 & -1.64074\end{array}$

H $\quad 1.99655 \quad .80902-1.93449$

$\begin{array}{llll}\text { C } & -3.95906 & 1.70577 & 1.08443\end{array}$

$\begin{array}{llll}\mathrm{H} & -5.01214 & 1.97862 & .97593\end{array}$

$\begin{array}{llll}\mathrm{H} & -3.60414 & 1.98073 & 2.07914\end{array}$

$\mathrm{H}-3.36403 \quad 2.20424 \quad .31728$

$\begin{array}{llll}\text { C } & -2.42963 & -5.46423 & 2.30973\end{array}$

$\begin{array}{llll}\text { C } & -2.76585 & -4.31257 & 3.02743\end{array}$

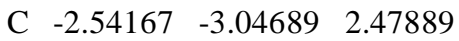

C $-1.97583 \quad-2.91495 \quad 1.20760$

$\begin{array}{llll}\text { C } & -1.65222 & -4.07404 & .48768\end{array}$

$\begin{array}{llll}\text { C } & -1.87264 & -5.34142 & 1.03291\end{array}$

$\begin{array}{llll}\mathrm{H} & -2.60674 & -6.44897 & 2.73683\end{array}$

$\begin{array}{llll}\mathrm{H} & -3.20869 & -4.39898 & 4.01758\end{array}$

$\begin{array}{llll}\mathrm{H} & -2.81217 & -2.15103 & 3.02520\end{array}$

$\begin{array}{llll}\mathrm{H} & -1.23421 & -3.98915 & -.51528\end{array}$

$\begin{array}{llll}\mathrm{H} & -1.61775 & -6.23016 & .45943\end{array}$

$\begin{array}{llll}\text { C } & .48050 & -.85787 & 1.80244\end{array}$

$\begin{array}{llll}\mathrm{O} & .22335 & -1.75352 & 2.74561\end{array}$

$\begin{array}{llll}\text { O } & 1.19304 & .11721 & 1.95284\end{array}$

$\begin{array}{llll}\text { C } & .79197 & -1.49796 & 4.05597\end{array}$

$\mathrm{H} \quad .41734 \quad-2.30049 \quad 4.68993$

H $\quad 1.88293 \quad-1.52214 \quad 4.00333$

$\begin{array}{llll}\mathrm{H} & .46050 & -.52571 & 4.42427\end{array}$
\end{abstract}

\section{Anti cisoid betaine (conformer}

3)

E(B3LYP 6-31G*) $=$

$\mathrm{E}\left(\mathrm{B} 3 \mathrm{LYP} 6-311+\mathrm{G}^{* *}\right)=$

$\mathrm{E}\left(\mathrm{B} 3 \mathrm{LYP} 6-31 \mathrm{G}^{*}\left(\mathrm{CH}_{3} \mathrm{CN}\right)\right)=$

$\mathrm{E}\left(\mathrm{B} 3 \mathrm{LYP} 6-311+\mathrm{G}^{* *}\left(\mathrm{CH}_{3} \mathrm{CN}\right)\right)=$

$\begin{array}{llll}\text { C } & -1.93013 & -1.727168 & .61067\end{array}$

$\begin{array}{llll}\mathrm{N} & -2.46900 & -.670475 & 1.43786\end{array}$

$\begin{array}{llll}\mathrm{H} & -2.43478 & -1.837652 & -.35875\end{array}$

$\begin{array}{llll}\mathrm{H} & .12842 & -2.301211 & -.04308\end{array}$

$\begin{array}{llll}\text { S } & -3.46029 & .342523 & .74764\end{array}$

$\begin{array}{llll}\text { O } & -3.11479 & .634707 & -.69387\end{array}$

$\begin{array}{llll}\mathrm{O} & -4.92208 & .042367 & .90521\end{array}$

$\begin{array}{llll}\text { C } & -.41240 & -1.406697 & .28109\end{array}$

$\begin{array}{llll}\text { S } & -.25214 & -.175891 & -1.11538\end{array}$

$\begin{array}{llll}\text { C } & 1.52419 & -.329702 & -1.50898\end{array}$

$\begin{array}{lllll}\mathrm{H} & 2.08024 & .127194 & -.68819\end{array}$

$\begin{array}{llll}\mathrm{H} & 1.80448 & -1.377859 & -1.64004\end{array}$

$\begin{array}{llll}\mathrm{H} & 1.69705 & .236242 & -2.42874\end{array}$

$\begin{array}{llll}\text { C } & -.97505 & -1.085015 & -2.51927\end{array}$

$\begin{array}{llll}\mathrm{H} & -.60968 & -2.114722 & -2.54810\end{array}$

H $\quad-2.05843 \quad-1.037736 \quad-2.40743$ 


$\begin{array}{cccc}\mathrm{H} & -.67620 & -.547853 & -3.42394 \\ \mathrm{C} & -3.19817 & 1.854848 & 1.68830 \\ \mathrm{H} & -3.88697 & 2.613911 & 1.30766 \\ \mathrm{H} & -3.40818 & 1.655283 & 2.74123 \\ \mathrm{H} & -2.16299 & 2.176960 & 1.56451 \\ \mathrm{C} & -2.23960 & -5.652628 & 2.45914 \\ \mathrm{C} & -2.52295 & -4.502787 & 3.20090 \\ \mathrm{C} & -2.40815 & -3.238538 & 2.61544 \\ \mathrm{C} & -2.00797 & -3.106177 & 1.28291 \\ \mathrm{C} & -1.72758 & -4.264111 & .54313 \\ \mathrm{C} & -1.84117 & -5.529174 & 1.12395 \\ \mathrm{H} & -2.33523 & -6.636606 & 2.91279 \\ \mathrm{H} & -2.84227 & -4.588587 & 4.23748 \\ \mathrm{H} & -2.64607 & -2.340156 & 3.17358 \\ \mathrm{H} & -1.43668 & -4.184215 & -.50369 \\ \mathrm{H} & -1.62900 & -6.417019 & .53242 \\ \mathrm{C} & .24671 & -.850614 & 1.54189 \\ \mathrm{O} & .46369 & .468196 & 1.48431 \\ \mathrm{O} & .51958 & -1.557323 & 2.48631 \\ \mathrm{C} & .95977 & 1.092706 & 2.69551 \\ \mathrm{H} & 1.08152 & 2.146576 & 2.44704 \\ \mathrm{H} & .22745 & .966211 & 3.49553 \\ \mathrm{H} & 1.91378 & .648855 & 2.98766\end{array}$

\section{Syn torsional TS}

$\mathrm{E}\left(\mathrm{B} 3 \mathrm{LYP} 6-31 \mathrm{G}^{*}\right)=-1658.694165$
$\mathrm{E}\left(\mathrm{B} 3 \mathrm{LYP} 6-311+\mathrm{G}^{* *}\right)=$
-1658.997855
$\mathrm{E}\left(\mathrm{B} 3 \mathrm{LYP} 6-31 \mathrm{G}^{*}\left(\mathrm{CH}_{3} \mathrm{CN}\right)\right)=$
-1658.751220
$\mathrm{E}\left(\mathrm{B} 3 \mathrm{LYP} 6-311+\mathrm{G}^{* *}\left(\mathrm{CH}_{3} \mathrm{CN}\right)\right)=$
-1659.057849

$\begin{array}{llll}\text { H } & -2.71447 & -.43777 & -2.02803\end{array}$

$\begin{array}{llll}\mathrm{H} & -3.42188 & -.01237 & 2.18454\end{array}$

H $-4.72794 \quad 2.04097 \quad 1.76827$

$\begin{array}{llll}\text { C } & -.04795 & -.10647 & -.34677\end{array}$

$\begin{array}{llll}\text { O } & -.04743 & -.32431 & -1.66162\end{array}$

$\begin{array}{llll}\text { O } & .26994 & .94636 & .17800\end{array}$

$\begin{array}{llll}\text { C } & .26231 & .80839 & -2.51140\end{array}$

H $\quad .23606 \quad .41736 \quad-3.52826$

H $\quad-.49087 \quad 1.58815 \quad-2.37794$

H $\quad 1.25361 \quad 1.19934 \quad-2.27253$

\section{Anti torsional TS}

$\mathrm{E}(\mathrm{B} 3 \mathrm{LYP}$ 6-31G*)

$-1658.681352$

$\mathrm{E}\left(\mathrm{B} 3 \mathrm{LYP} 6-311+\mathrm{G}^{* *}\right)=$

$-1658.986429$

$\mathrm{E}\left(\mathrm{B} 3 \mathrm{LYP} 6-31 \mathrm{G}^{*}\left(\mathrm{CH}_{3} \mathrm{CN}\right)\right)=$ $-1658.752933$

$\mathrm{E}\left(\mathrm{B} 3 \mathrm{LYP} 6-311+\mathrm{G}^{* *}\left(\mathrm{CH}_{3} \mathrm{CN}\right)\right)=$ $-1659.062296$

$\begin{array}{llll}C & -1.74414 & -1.59814 & 50107\end{array}$

$\begin{array}{llll}\mathrm{N} & -2.34792 & -.29535 & .68314\end{array}$

H $\quad-2.02881 \quad-2.04276 \quad-.46415$

$\begin{array}{llll}\mathrm{H} & .00371 & -.26435 & .61915\end{array}$

$\begin{array}{llll}\mathrm{S} & -3.79263 & -.06978 & .06005\end{array}$

$\begin{array}{llll}\mathrm{O} & -4.02159 & 1.40034 & -.03425\end{array}$

$\begin{array}{llll}\text { O } & -4.04457 & -.83892 & -1.19756\end{array}$

$\begin{array}{llll}\text { C } & -.17288 & -1.31547 & .38414\end{array}$

$\begin{array}{llll}\mathrm{S} & .87935 & -2.32214 & 1.54952\end{array}$

C $\quad 2.58645 \quad-1.94163 \quad 1.01494$

H $\quad 2.78282 \quad-2.51032 \quad .10657$

$\begin{array}{llll}\mathrm{H} & 2.70748 & -.86729 & .85469\end{array}$

H $\quad 3.24027 \quad-2.28676 \quad 1.82062$

$\begin{array}{llll}\text { C } & .81836 & -1.31209 & 3.06858\end{array}$

$\begin{array}{llll}\mathrm{H} & 1.10097 & -.28112 & 2.84303\end{array}$

H $\quad-.19361 \quad-1.36641 \quad 3.46914$

$\begin{array}{llll}\mathrm{H} & 1.52211 & -1.76579 & 3.77139\end{array}$

$\begin{array}{llll}\text { C } & -5.08810 & -.62845 & 1.19961\end{array}$

$\begin{array}{llll}\mathrm{H} & -6.05603 & -.36742 & .76230\end{array}$

$\begin{array}{llll}\mathrm{H} & -5.01629 & -1.70863 & 1.33882\end{array}$

H $-4.96120 \quad-.11764 \quad 2.15692$

C $\quad-3.05688-4.53111 \quad 3.42785$

C $\quad-2.98174 \quad-3.17998 \quad 3.77820$

C $\quad \begin{array}{llll}\text { C.52962 } & -2.23958 & 2.85011\end{array}$

$\begin{array}{llll}\text { C } & -2.13517 & -2.63559 & 1.56437\end{array}$

$\begin{array}{llll}\text { C } & -2.20742 & -3.99414 & 1.22473\end{array}$

C $-2.66705 \quad-4.93642 \quad 2.14783$

H $\quad-3.43027 \quad-5.26175 \quad 4.14124$

H $\quad-3.29421 \quad-2.85115 \quad 4.76673$

$\mathrm{H} \quad-2.52558 \quad-1.18252 \quad 3.10031$

$\begin{array}{llll}\mathrm{H} & -1.92610 & -4.31199 & .22216\end{array}$

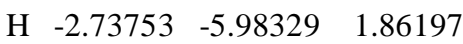

C $\quad .28646 \quad-1.65762-1.02625$

$\begin{array}{lllll}\text { O } & .07901 & -.64667 & -1.85902\end{array}$

$\begin{array}{llll}\mathrm{O} & .73796 & -2.74659 & -1.33848\end{array}$

$\begin{array}{llll}\text { C } & .39424 & -.87655 & -3.25966\end{array}$

$\mathrm{H} \quad \begin{array}{llll}17566 & .06717 & -3.75806\end{array}$

H $1.44993 \quad-1.13580 \quad-3.36542$

H $\quad-.23051 \quad-1.67864 \quad-3.65736$

Syn transoid betaine
$\mathrm{E}\left(\mathrm{B} 3 \mathrm{LYP} 6-31 \mathrm{G}^{*}\right)=-1658.659250$

$\mathrm{E}($ B3LYP 6-311+G**) =

$-1659.002198$

$\mathrm{E}\left(\mathrm{B} 3 \mathrm{LYP} 6-31 \mathrm{G} *\left(\mathrm{CH}_{3} \mathrm{CN}\right)\right)=$

$-1658.757218$

$\mathrm{E}\left(\mathrm{B} 3 \mathrm{LYP} 6-311+\mathrm{G}^{* *}\left(\mathrm{CH}_{3} \mathrm{CN}\right)\right)=$ $-1659.063806$

$\begin{array}{llll}\text { C } & -1.66497 & -1.60470 & .32816\end{array}$

$\begin{array}{llll}\mathrm{N} & -2.34729 & -.33048 & .24858\end{array}$

H $-1.83284 \quad-2.23666 \quad-.55963$

H $\quad .14588 \quad-.60365 \quad-.38399$

$\begin{array}{llll}\mathrm{S} & -2.34651 & .35801 & -1.18169\end{array}$

$\begin{array}{lllll}\text { O } & -.97509 & .59321 & -1.76580\end{array}$

$\begin{array}{lllll}\text { O } & -3.25667 & -.27078 & -2.19726\end{array}$

$\begin{array}{llll}\text { C } & -.08249 & -1.35093 & .38334\end{array}$

$\begin{array}{llll}\mathrm{S} & .87778 & -2.89203 & -.04737\end{array}$

C $\quad .40424 \quad-3.14146 \quad-1.79392$

$\mathrm{H} \quad-.57922 \quad-3.61301-1.81950$

H $\quad .39617-2.18818-2.32685$

H $\quad 1.14297 \quad-3.82391 \quad-2.22222$

$\begin{array}{llll}\text { C } & 2.58289 & -2.27726 & -.26463\end{array}$

H $\quad 2.58347 \quad-1.38673 \quad-.89769$

$\begin{array}{llll}\mathrm{H} & 2.98115 & -2.06443 & .72755\end{array}$

H $\quad 3.14820 \quad-3.08684 \quad-.73316$

$\begin{array}{llll}\text { C } & -3.04570 & 1.97194 & -.81386\end{array}$

H $-3.08968 \quad 2.52744 \quad-1.75434$

H $-4.05078 \quad 1.84382-.40746$

$\begin{array}{llll}\mathrm{H} & -2.40797 & 2.48834 & -.09411\end{array}$

C $\quad \begin{array}{llll}-3.05722 & -3.86709 & 3.76432\end{array}$

$\begin{array}{llll}\text { C } & -3.01129 & -2.47025 & 3.81293\end{array}$

C $\quad-2.56221 \quad-1.74223 \quad 2.70947$

$\begin{array}{llll}\text { C } & -2.15001 & -2.39767 & 1.54031\end{array}$

$\begin{array}{llll}\text { C } & -2.20429 & -3.79729 & 1.49889\end{array}$

$\begin{array}{llll}\text { C } & -2.65371 & -4.52952 & 2.60250\end{array}$

H $-3.41702 \quad-4.43335 \quad 4.62030$

$\begin{array}{llll}\mathrm{H} & -3.33877 & -1.94511 & 4.70739\end{array}$

$\begin{array}{llll}\mathrm{H} & -2.56075 & -.65769 & 2.73112\end{array}$

H $-1.92466 \quad-4.32621 \quad .58886$

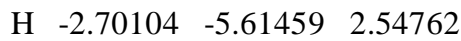

$\begin{array}{llll}\text { C } & .42975 & -.91582 & 1.74564\end{array}$

$\begin{array}{llll}\text { O } & .02538 & .30810 & 2.05935\end{array}$

$\begin{array}{llll}\text { O } & 1.13166 & -1.62097 & 2.45363\end{array}$

$\begin{array}{llll}\text { C } & .40879 & .80197 & 3.36786\end{array}$

$\mathrm{H} \quad-.02866 \quad 1.79789 \quad 3.43532$

$\begin{array}{llll}\mathrm{H} & .00535 & .15002 & 4.14584\end{array}$

H $1.49701 \quad .85019 \quad 3.44872$

\section{Syn transoid betaine (conformer 1)}

$\mathrm{E}\left(\mathrm{B} 3 \mathrm{LYP} 6-31 \mathrm{G}^{*}\right)=-1658.6264408$

$\mathrm{E}\left(\mathrm{B} 3 \mathrm{LYP} 6-311+\mathrm{G}^{* *}\right)=$

$-1658.994574$

$\mathrm{E}\left(\mathrm{B} 3 \mathrm{LYP} 6-31 \mathrm{G}^{*}\left(\mathrm{CH}_{3} \mathrm{CN}\right)\right)=$

$-1658.754256$

$\mathrm{E}\left(\mathrm{B} 3 \mathrm{LYP} 6-311+\mathrm{G} * *\left(\mathrm{CH}_{3} \mathrm{CN}\right)\right)=$ $-1659.060932$

$\begin{array}{llll}\text { C } & -1.65710 & -1.63657 & .31512\end{array}$

$\begin{array}{llll}\mathrm{N} & -2.24217 & -.31240 & .45565\end{array}$ 
H $-1.77789-2.06581 \quad-.69143$

H $\quad .29130 \quad-1.00514 \quad-.41028$

$\begin{array}{llll}\text { S } & -2.25523 & .57630 & -.86101\end{array}$

$\begin{array}{lllll}\text { O } & -.91345 & 1.07126 & -1.32521\end{array}$

$\begin{array}{lllll}\text { O } & -3.03407 & -.01638 & -2.00315\end{array}$

$\begin{array}{llll}\text { C } & -.09541 & -1.47423 & .49785\end{array}$

$\begin{array}{llll}\text { S } & .79557 & -3.12034 & .51063\end{array}$

$\begin{array}{llll}\text { C } & 2.51276 & -2.65345 & .10690\end{array}$

H $\quad 2.52258 \quad-2.32488 \quad-.93509$

H $2.86194-1.86064 \quad .77221$

$\begin{array}{llll}\mathrm{H} & 3.12558 & -3.55208 & .21814\end{array}$

C $.96043 \quad-3.66076 \quad 2.24703$

H $1.54299-2.941492 .82262$

H $\quad-.04413 \quad-3.76254 \quad 2.65728$

H 1.45449 -4.63547 2.20700

$\begin{array}{llll}\text { C } & -3.16280 & 2.02694 & -.30824\end{array}$

H $-3.23632 \quad 2.70491-1.16281$

$\begin{array}{llll}\mathrm{H} & -4.15867 & 1.73302 & .02692\end{array}$

$\begin{array}{llll}\mathrm{H} & -2.61426 & 2.50660 & .50437\end{array}$

$\begin{array}{llll}\text { C } & -3.53900 & -4.43921 & 3.04533\end{array}$

$\begin{array}{llll}\text { C } & -3.41601 & -3.09057 & 3.39928\end{array}$

$\begin{array}{llll}\text { C } & -2.80194 & -2.18711 & 2.53152\end{array}$

C -2.30282 $-2.61486 \quad 1.29284$

$\begin{array}{lll}\text { C }-2.44180 & -3.96529 & .94007\end{array}$

C $-3.05064-4.87539 \quad 1.81181$

H $-4.02598 \quad-5.13917 \quad 3.72048$

H $-3.80935-2.73893 \quad 4.35058$

H $-2.72971 \quad-1.13729 \quad 2.79008$

H $-2.09622-4.30507 \quad-.03457$

$\begin{array}{llll}\mathrm{H} & -3.15830 & -5.91660 & 1.51687\end{array}$

C $\quad .26236 \quad-.60384 \quad 1.69909$

$\begin{array}{llll}\mathrm{O} & .90196 & .50435 & 1.31550\end{array}$

$\begin{array}{lllll}\text { O } & .01743 & -.89108 & 2.85408\end{array}$

$\begin{array}{llll}\text { C } & 1.18190 & 1.47020 & 2.35587\end{array}$

H $1.67811 \quad 2.30062 \quad 1.85414$

H $\quad .24697 \quad 1.80122 \quad 2.81340$

H $1.83169 \quad 1.03398 \quad 3.11781$

\section{Syn transoid betaine (conformer 2)}

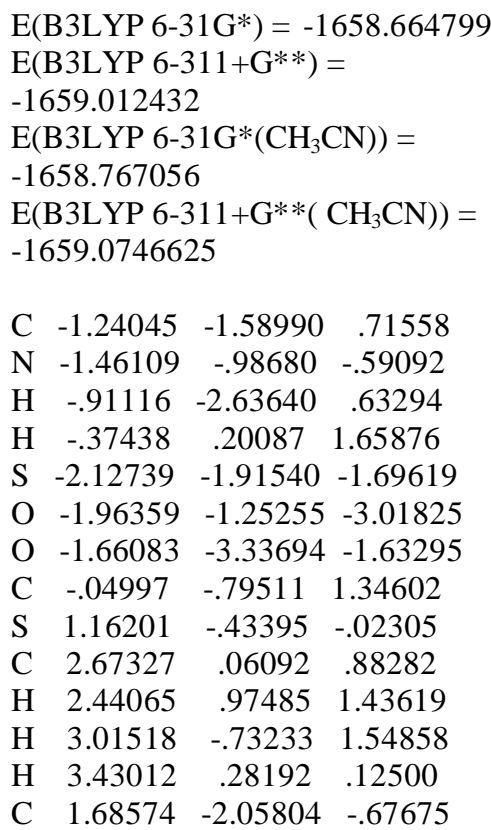

$\begin{array}{llll}\mathrm{H} & 2.03876 & -2.68991 & .13754\end{array}$

$\mathrm{H} \quad \begin{array}{llll}82809 & -2.50411 & -1.18259\end{array}$

H $\quad 2.48238-1.85012-1.39594$

$\begin{array}{llll}\text { C } & -3.91901 & -1.99915 & -1.44034\end{array}$

$\begin{array}{llll}\mathrm{H} & -4.35265 & -2.58384 & -2.25654\end{array}$

H $\quad-4.12396-2.48024 \quad-.48097$

H $\quad-4.32165 \quad-.98359-1.44678$

$\begin{array}{llll}\text { C } & -4.83863 & -1.56859 & 3.14171\end{array}$

$\begin{array}{llll}\text { C } & -4.34629 & -.37178 & 2.60645\end{array}$

$\begin{array}{llll}\text { C } & -3.17396 & -.36984 & 1.85230\end{array}$

$\begin{array}{llll}\text { C } & -2.47079 & -1.56219 & 1.62697\end{array}$

$\begin{array}{llll}\text { C } & -2.96220 & -2.75326 & 2.17340\end{array}$

$\begin{array}{llll}\text { C } & -4.14398 & -2.75955 & 2.92407\end{array}$

$\begin{array}{llll}\mathrm{H} & -5.76172 & -1.56950 & 3.71677\end{array}$

$\begin{array}{llll}\mathrm{H} & -4.88604 & .55898 & 2.76606\end{array}$

$\begin{array}{llll}\mathrm{H} & -2.81739 & .55803 & 1.41320\end{array}$

$\begin{array}{llll}\mathrm{H} & -2.43180 & -3.68714 & 1.99318\end{array}$

$\begin{array}{llll}\mathrm{H} & -4.52311 & -3.69634 & 3.32618\end{array}$

$\begin{array}{llll}\text { C } & .55170 & -1.55784 & 2.52007\end{array}$

$\begin{array}{llll}\mathrm{O} & .11489 & -1.07240 & 3.68198\end{array}$

$\begin{array}{llll}\text { O } & 1.29368 & -2.51777 & 2.40675\end{array}$

C $\quad .51126 \quad-1.80741 \quad 4.86993$

H $\quad \begin{array}{llll}\text { H } & .86555 & -1.25144 & 5.70429\end{array}$

$\begin{array}{llll}\mathrm{H} & .10684 & -2.82159 & 4.83151\end{array}$

H $\quad 1.59991 \quad-1.84446 \quad 4.94474$

\section{Syn transoid betaine (conformer} 3)

$\mathrm{E}\left(\mathrm{B} 3 \mathrm{LYP} 6-31 \mathrm{G}^{*}\right)=-1658.682090$

$\mathrm{E}\left(\mathrm{B} 3 \mathrm{LYP} 6-311+\mathrm{G}^{* *}\right)=$

$-1658.983969$

$\mathrm{E}\left(\mathrm{B} 3 \mathrm{LYP} 6-31 \mathrm{G}^{*}\left(\mathrm{CH}_{3} \mathrm{CN}\right)\right)=$ $-1658.750494$

$\mathrm{E}\left(\mathrm{B} 3 \mathrm{LYP} 6-311+\mathrm{G} * *\left(\mathrm{CH}_{3} \mathrm{CN}\right)\right)=$ $-1659.060261$

$\begin{array}{llll}\mathrm{C} & -1.08833 & -1.54582 & .16469 \\ \mathrm{~N} & -1.59814 & -.21844 & -.13623 \\ \mathrm{H} & -.92900 & -2.13365 & -.75159 \\ \mathrm{H} & .87627 & -.71929 & -.09868 \\ \mathrm{~S} & -2.73934 & -.12263 & -1.23641 \\ \mathrm{O} & -2.73754 & 1.26157 & -1.78940 \\ \mathrm{O} & -2.69276 & -1.22000 & -2.25467 \\ \mathrm{C} & .35545 & -1.25528 & .69869 \\ \mathrm{~S} & 1.35379 & -2.83268 & .80407 \\ \mathrm{C} & 3.05771 & -2.20872 & .99399 \\ \mathrm{H} & 3.31260 & -1.66767 & .07979 \\ \mathrm{H} & 3.13902 & -1.56181 & 1.87036 \\ \mathrm{H} & 3.70541 & -3.08295 & 1.10023 \\ \mathrm{C} & 1.05699 & -3.59522 & 2.43345 \\ \mathrm{H} & 1.29818 & -2.89852 & 3.23611 \\ \mathrm{H} & .00661 & -3.88672 & 2.47117 \\ \mathrm{H} & 1.69670 & -4.48205 & 2.46319 \\ \mathrm{C} & -4.38649 & -.26349 & -.48749 \\ \mathrm{H} & -5.12470 & -.11175 & -1.27977 \\ \mathrm{H} & -4.50661 & -1.25072 & -.03912 \\ \mathrm{H} & -4.49668 & .51050 & .27535 \\ \mathrm{C} & -3.84289 & -3.90401 & 2.56063 \\ \mathrm{C} & -3.63901 & -2.55113 & 2.85698 \\ \mathrm{C} & -2.72573 & -1.79912 & 2.11973 \\ \mathrm{C} & -1.99605 & -2.38354 & 1.07220 \\ \mathrm{C} & -2.21099 & -3.73723 & .78036\end{array}$ $\begin{array}{llll}\text { C } & -3.12363 & -4.49691 & 1.52125\end{array}$

H $-4.56902-4.48382 \quad 3.12576$

H $\quad-4.20481 \quad-2.07606 \quad 3.65566$

$\begin{array}{llll}\mathrm{H} & -2.60087 & -.74212 & 2.32878\end{array}$

H $-1.68487-4.19476-.05577$

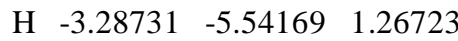

$\begin{array}{llll}\text { C } & .44777 & -.45212 & 1.99441\end{array}$

$\begin{array}{llll}\mathrm{O} & 1.15731 & .66667 & 1.82615\end{array}$

$\begin{array}{lllll}\text { O } & -.02493 & -.81019 & 3.05564\end{array}$

$\begin{array}{llll}\text { C } & 1.30669 & 1.51574 & 2.99427\end{array}$

$\begin{array}{llll}\mathrm{H} & 1.90011 & 2.36539 & 2.65783\end{array}$

H $\quad .32839 \quad 1.84555 \quad 3.34913$

$\begin{array}{llll}\mathrm{H} & 1.82263 & .97430 & 3.79093\end{array}$

\section{Syn transoid betaine (conformer 4)}

$\mathrm{E}($ B3LYP 6-31G*) $=-1658.666774$

$\mathrm{E}\left(\right.$ B3LYP $\left.6-311+\mathrm{G}^{* *}\right)=$

$-1658.997598$

$\mathrm{E}\left(\mathrm{B} 3 \mathrm{LYP} 6-31 \mathrm{G}^{*}\left(\mathrm{CH}_{3} \mathrm{CN}\right)\right)=$

$-1658.753455$

$\mathrm{E}\left(\mathrm{B} 3 \mathrm{LYP} 6-311+\mathrm{G}^{* *}\left(\mathrm{CH}_{3} \mathrm{CN}\right)\right)=$ $-1659.060864$

$\begin{array}{llll}\text { C } & -1.61414 & -1.62583 & .40988\end{array}$

$\begin{array}{llll}\mathrm{N} & -2.33744 & -.37832 & .27530\end{array}$

$\mathrm{H}-1.69311 \quad-2.26059 \quad-.48939$

$\mathrm{H} \quad .18630 \quad-.62128 \quad-.24683$

$\begin{array}{llll}\text { S } & -2.24910 & .28710 & -1.16703\end{array}$

$\begin{array}{lllll}\text { O } & -.87907 & .78732 & -1.54985\end{array}$

$\begin{array}{lllll}\mathrm{O} & -2.84782 & -.53206 & -2.27597\end{array}$

$\begin{array}{llll}\text { C } & -.03336 & -1.35280 & .54135\end{array}$

$\begin{array}{llll}\mathrm{S} & .92393 & -2.83506 & -.04870\end{array}$

$\begin{array}{llll}\text { C } & 2.65624 & -2.27060 & -.07254\end{array}$

$\begin{array}{llll}\mathrm{H} & 2.72935 & -1.46845 & -.81050\end{array}$

$\begin{array}{llll}\mathrm{H} & 2.95661 & -1.93326 & .92040\end{array}$

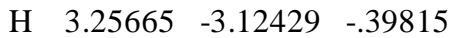

$\begin{array}{llll}\text { C } & .96678 & -4.11040 & 1.25804\end{array}$

$\begin{array}{llll}\mathrm{H} & 1.37823 & -3.69295 & 2.17476\end{array}$

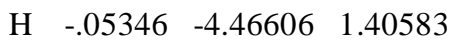

$\begin{array}{llll}\mathrm{H} & 1.58675 & -4.91726 & .85729\end{array}$

$\begin{array}{llll}\text { C } & -3.28910 & 1.73952 & -.98274\end{array}$

$\begin{array}{llll}\mathrm{H} & -3.26924 & 2.26626 & -1.94053\end{array}$

$\begin{array}{lllll}\mathrm{H} & -4.30883 & 1.42945 & -.74753\end{array}$

$\begin{array}{llll}\mathrm{H} & -2.88974 & 2.37591 & -.19128\end{array}$

$\begin{array}{llll}\text { C } & -3.35705 & -3.88338 & 3.68289\end{array}$

$\begin{array}{llll}\text { C } & -2.97385 & -2.55149 & 3.87386\end{array}$

$\begin{array}{llll}\text { C } & -2.39746 & -1.82846 & 2.82866\end{array}$

$\begin{array}{llll}\text { C } & -2.19172 & -2.42340 & 1.57462\end{array}$

$\begin{array}{llll}\text { C } & -2.58976 & -3.75441 & 1.38939\end{array}$

$\begin{array}{llll}\text { C } & -3.16535 & -4.48317 & 2.43641\end{array}$

$\begin{array}{llll}\mathrm{H} & -3.81323 & -4.44297 & 4.49614\end{array}$

$\begin{array}{llll}\mathrm{H} & -3.13571 & -2.07055 & 4.83580\end{array}$

$\begin{array}{llll}\mathrm{H} & -2.14540 & -.78217 & 2.97125\end{array}$

$\begin{array}{llll}\mathrm{H} & -2.47417 & -4.22110 & .41222\end{array}$

$\begin{array}{llll}\mathrm{H} & -3.47533 & -5.51233 & 2.27068\end{array}$

$\begin{array}{llll}\text { C } & .45808 & -.80733 & 1.87459\end{array}$

$\begin{array}{llll}\text { O } & .05782 & .45105 & 2.04322\end{array}$

$\begin{array}{llll}\text { O } & 1.13179 & -1.42436 & 2.68203\end{array}$

$\begin{array}{llll}\text { C } & .42070 & 1.07882 & 3.29911\end{array}$

$\begin{array}{llll}\mathrm{H} & -.00148 & 2.08159 & 3.24843\end{array}$

$\begin{array}{llll}\mathrm{H} & -.00759 & .52352 & 4.13655\end{array}$ 
H $\quad 1.50768 \quad 1.12254 \quad 3.39832$

Anti transoid betaine

$\mathrm{E}\left(\mathrm{B} 3 \mathrm{LYP} 6-31 \mathrm{G}^{*}\right)=-1658.688730$

$\mathrm{E}\left(\mathrm{B} 3 \mathrm{LYP} 6-311+\mathrm{G}^{* *}\right)=$

$-1658.991352$

$\mathrm{E}\left(\mathrm{B} 3 \mathrm{LYP} 6-31 \mathrm{G}^{*}\left(\mathrm{CH}_{3} \mathrm{CN}\right)\right)=$

$-1658.759826$

$\mathrm{E}\left(\mathrm{B} 3 \mathrm{LYP} 6-311+\mathrm{G}^{* *}\left(\mathrm{CH}_{3} \mathrm{CN}\right)\right)=$ $-1659.070004$

$\begin{array}{lclc}\mathrm{C} & -1.69395 & -1.69862 & .310717 \\ \mathrm{~N} & -2.30936 & -.37597 & .395486 \\ \mathrm{H} & -1.89999 & -2.17862 & -.657066 \\ \mathrm{H} & .07368 & -.84005 & 1.268864 \\ \mathrm{~S} & -3.85500 & -.28385 & .032992 \\ \mathrm{O} & -4.16669 & 1.13005 & -.322011 \\ \mathrm{O} & -4.31428 & -1.30524 & -.959666 \\ \mathrm{C} & -.17636 & -1.36101 & .341056 \\ \mathrm{~S} & .86378 & -2.90447 & .441455 \\ \mathrm{C} & .47515 & -3.85797 & -1.064340 \\ \mathrm{H} & -.52091 & -4.28425 & -.930451 \\ \mathrm{H} & .51526 & -3.21100 & -1.941108 \\ \mathrm{H} & 1.21531 & -4.66088 & -1.117593 \\ \mathrm{C} & 2.54292 & -2.28897 & .084814 \\ \mathrm{H} & 2.59512 & -1.87509 & -.924484 \\ \mathrm{H} & 2.78238 & -1.53613 & .840079 \\ \mathrm{H} & 3.21904 & -3.14158 & .187533 \\ \mathrm{C} & -4.86995 & -.58383 & 1.507565 \\ \mathrm{H} & -5.91790 & -.43201 & 1.232908 \\ \mathrm{H} & -4.71745 & -1.60616 & 1.857084 \\ \mathrm{H} & -4.58191 & .12796 & 2.284240 \\ \mathrm{C} & -3.17692 & -4.33109 & 3.419799 \\ \mathrm{C} & -2.44633 & -3.18715 & 3.765030 \\ \mathrm{C} & -1.92927 & -2.35828 & 2.769831 \\ \mathrm{C} & -2.12684 & -2.66324 & 1.413319 \\ \mathrm{C} & -2.84976 & -3.81557 & 1.077693 \\ \mathrm{C} & -3.37600 & -4.64449 & 2.073807 \\ \mathrm{H} & -3.59346 & -4.96711 & 4.197098 \\ \mathrm{H} & -2.29248 & -2.93531 & 4.811891 \\ \mathrm{H} & -1.39562 & -1.45275 & 3.049905 \\ \mathrm{H} & -3.03501 & -4.04519 & .030317 \\ \mathrm{H} & -3.95054 & -5.52472 & 1.794709 \\ \mathrm{C} & .20548 & -.50180 & -.863595 \\ \mathrm{O} & .69279 & .68030 & -.491014 \\ \mathrm{O} & .10473 & -.87737 & -2.016464 \\ \mathrm{C} & 1.02699 & 1.60771 & -1.557259 \\ \mathrm{H} & 1.38537 & 2.50547 & -1.053908 \\ \mathrm{H} & 1.80551 & 1.18386 & -2.195603 \\ \mathrm{H} & .13735 & 1.82768 & -2.151233\end{array}$

\section{Anti transoid betaine (conformer 1)}

$\mathrm{E}\left(\mathrm{B} 3 \mathrm{LYP} 6-31 \mathrm{G}^{*}\right)=-1658.648275$

$\mathrm{E}\left(\right.$ B3LYP $\left.6-311+\mathrm{G}^{* *}\right)=$

$-1658.990504$

$\mathrm{E}\left(\mathrm{B} 3 \mathrm{LYP} 6-31 \mathrm{G}^{*}\left(\mathrm{CH}_{3} \mathrm{CN}\right)\right)=$

$-1658.759863$

$\mathrm{E}\left(\mathrm{B} 3 \mathrm{LYP} 6-311+\mathrm{G}^{* *}\left(\mathrm{CH}_{3} \mathrm{CN}\right)\right)=$ $-1659.068300$

$\begin{array}{lccc}\text { C } & -1.79850 & -1.78448 & .50700 \\ \text { N } & -2.44964 & -.49285 & .67520 \\ \text { H } & -2.05069 & -2.24138 & -.45843 \\ \text { H } & .01870 & -1.00371 & 1.44653 \\ \text { S } & -3.69595 & -.16506 & -.25713 \\ \text { O } & -4.07231 & -1.27579 & -1.18591 \\ \text { O } & -4.82529 & .38413 & .55090 \\ \text { C } & -.28221 & -1.42809 & .48557 \\ \text { S } & .79014 & -2.95258 & .35287 \\ \text { C } & .24979 & -3.77346 & -1.18385 \\ \text { H } & -.70700 & -4.25643 & -.97597 \\ \text { H } & .16017 & -3.04005 & -1.98574 \\ \text { H } & 1.00240 & -4.53291 & -1.41162 \\ \text { C } & 2.41689 & -2.27815 & -.12151 \\ \text { H } & 2.35282 & -1.76405 & -1.08305 \\ \text { H } & 2.73347 & -1.60047 & .67531 \\ \text { H } & 3.10614 & -3.12414 & -.18095 \\ \text { C } & -3.23011 & 1.22509 & -1.31866 \\ \text { H } & -4.11119 & 1.53087 & -1.88974 \\ \text { H } & -2.89412 & 2.05251 & -.68906 \\ \text { H } & -2.43357 & .90764 & -1.99421 \\ \text { C } & -2.83286 & -4.66369 & 3.59650 \\ \text { C } & -2.17630 & -3.47602 & 3.94018 \\ \text { C } & -1.83496 & -2.55117 & 2.95259 \\ \text { C } & -2.13701 & -2.79735 & 1.60341 \\ \text { C } & -2.79832 & -3.98759 & 1.27136 \\ \text { C } & -3.14702 & -4.91503 & 2.25943 \\ \text { H } & -3.10216 & -5.38195 & 4.36754 \\ \text { H } & -1.93528 & -3.26845 & 4.98042 \\ \text { H } & -1.34622 & -1.62302 & 3.24030 \\ \text { H } & -3.06812 & -4.18015 & .23465 \\ \text { H } & -3.66810 & -5.82874 & 1.98273 \\ \text { C } & -.00441 & -.42706 & -.63325 \\ \text { O } & .49898 & .71049 & -.15633 \\ \text { O } & -.20803 & -.65514 & -1.81206 \\ \text { C } & .72393 & 1.77931 & -1.11210 \\ \text { H } & 1.14353 & 2.59929 & -.52989 \\ \text { H } & 1.42121 & 1.45557 & -1.88741 \\ \text { H } & -.22376 & 2.07719 & -1.56555\end{array}$

$\begin{array}{llll}\mathrm{H} & 2.65308 & -1.69200 & -.04406\end{array}$

$\begin{array}{llll}\mathrm{H} & 2.88669 & -3.37746 & -.63067\end{array}$

$\begin{array}{llll}\text { C } & 1.23229 & -3.51633 & 1.81696\end{array}$

$\begin{array}{llll}\mathrm{H} & 1.81208 & -2.69620 & 2.24682\end{array}$

H $\quad .34940 \quad-3.73150 \quad 2.41983$

$\begin{array}{lrrr}\mathrm{H} & 1.84174 & -4.41731 & 1.70900\end{array}$

C $\quad-3.21381 \quad 1.26603 \quad-1.27176$

$\begin{array}{lllll}\mathrm{H} & -4.11045 & 1.60767 & -1.79646\end{array}$

$\begin{array}{llll}\mathrm{H} & -2.79288 & 2.08812 & -.68800\end{array}$

$\begin{array}{llll}\mathrm{H} & -2.48133 & .88584 & -1.98627\end{array}$

$\begin{array}{llll}\text { C } & -2.77879 & -4.60591 & 3.69195\end{array}$

$\begin{array}{llll}\text { C } & -2.38703 & -3.30178 & 4.01928\end{array}$

$\begin{array}{llll}\text { C } & -2.08657 & -2.38520 & 3.01068\end{array}$

$\begin{array}{llll}\text { C } & -2.16340 & -2.75638 & 1.65883\end{array}$

$\begin{array}{llll}\text { C } & -2.55919 & -4.06312 & 1.34163\end{array}$

$\begin{array}{llll}\text { C } & -2.86778 & -4.98350 & 2.35065\end{array}$

$\begin{array}{llll}\mathrm{H} & -3.02062 & -5.31705 & 4.47860\end{array}$

$\begin{array}{llll}\mathrm{H} & -2.32452 & -2.99775 & 5.06183\end{array}$

H $\quad-1.80850 \quad-1.36809 \quad 3.27077$

$\begin{array}{llll}\mathrm{H} & -2.64465 & -4.35894 & .29779\end{array}$

$\begin{array}{llll}\mathrm{H} & -3.18490 & -5.98964 & 2.08630\end{array}$

$\begin{array}{llll}\text { C } & -.05454 & -.52906 & -.71487\end{array}$

$\begin{array}{llll}\mathrm{O} & .50216 & .61490 & -.32164\end{array}$

$\begin{array}{llll}\mathrm{O} & -.33330 & -.81191 & -1.86500\end{array}$

$\begin{array}{llll}\text { C } & .70000 & 1.62630 & -1.34428\end{array}$

$\begin{array}{llll}\mathrm{H} & 1.18833 & 2.45690 & -.83495\end{array}$

$\begin{array}{lllll}\mathrm{H} & 1.32811 & 1.23534 & -2.14721\end{array}$

$\begin{array}{llll}\mathrm{H} & -.26516 & 1.93930 & -1.74771\end{array}$

\section{Syn elimination TS}

$\mathrm{E}\left(\mathrm{B} 3 \mathrm{LYP} 6-31 \mathrm{G}^{*}\right)=-1658.703525$

$\mathrm{E}\left(\mathrm{B} 3 \mathrm{LYP} 6-311+\mathrm{G}^{* *}\right)=$

$-1659.000369$

$\mathrm{E}\left(\mathrm{B} 3 \mathrm{LYP} 6-31 \mathrm{G} *\left(\mathrm{CH}_{3} \mathrm{CN}\right)\right)=$

$-1658.745275$

$\mathrm{E}\left(\mathrm{B} 3 \mathrm{LYP} 6-311+\mathrm{G}^{* *}\left(\mathrm{CH}_{3} \mathrm{CN}\right)\right)=$ $-1659.049195$

$\begin{array}{lccc}\mathrm{C} & -1.50535 & -1.67267 & .33627 \\ \mathrm{~N} & -1.90538 & -.26578 & .44420 \\ \mathrm{H} & -1.61300 & -2.09006 & -.67145 \\ \mathrm{H} & .36053 & -.82362 & -.40897 \\ \mathrm{~S} & -2.36838 & .44731 & -.94957 \\ \mathrm{O} & -2.24568 & 1.91292 & -.77489 \\ \mathrm{O} & -1.70987 & -.12694 & -2.15603 \\ \mathrm{C} & -.07739 & -1.23199 & .49639 \\ \mathrm{~S} & 1.32801 & -3.09046 & .46373 \\ \mathrm{C} & 2.96594 & -2.37385 & .08999 \\ \mathrm{H} & 2.92527 & -1.99559 & -.93468 \\ \mathrm{H} & 3.20128 & -1.55920 & .77848 \\ \mathrm{H} & 3.71988 & -3.16344 & .15649 \\ \mathrm{C} & 1.55445 & -3.65839 & 2.18524 \\ \mathrm{H} & 1.80143 & -2.82679 & 2.84904 \\ \mathrm{H} & .61251 & -4.11473 & 2.49787 \\ \mathrm{H} & 2.35066 & -4.40834 & 2.20040 \\ \mathrm{C} & -4.13265 & .09445 & -1.11742 \\ \mathrm{H} & -4.48580 & .59250 & -2.02528 \\ \mathrm{H} & -4.27737 & -.98530 & -1.19997 \\ \mathrm{H} & -4.65410 & .48556 & -.24139 \\ \mathrm{C} & -3.50764 & -4.38994 & 3.04483 \\ \mathrm{C} & -3.58584 & -3.01010 & 3.25797 \\ \mathrm{C} & -2.92059 & -2.12633 & 2.40629\end{array}$




$\begin{array}{cccc}\mathrm{C} & -2.17374 & -2.60994 & 1.32651 \\ \mathrm{C} & -2.11127 & -3.99344 & 1.10805 \\ \mathrm{C} & -2.76608 & -4.88122 & 1.96679 \\ \mathrm{H} & -4.03242 & -5.07591 & 3.70557 \\ \mathrm{H} & -4.17073 & -2.61825 & 4.08724 \\ \mathrm{H} & -2.98762 & -1.05601 & 2.56327 \\ \mathrm{H} & -1.57035 & -4.37874 & .24539 \\ \mathrm{H} & -2.71417 & -5.95125 & 1.77997 \\ \mathrm{C} & .38955 & -.59925 & 1.77881 \\ \mathrm{O} & 1.39877 & .25678 & 1.54170 \\ \mathrm{O} & -.02887 & -.86909 & 2.88638 \\ \mathrm{C} & 1.96426 & .91477 & 2.70274 \\ \mathrm{H} & 2.77710 & 1.53049 & 2.31800 \\ \mathrm{H} & 1.20838 & 1.53549 & 3.18917 \\ \mathrm{H} & 2.34393 & .17684 & 3.41334\end{array}$

Syn elimination TS (conformer 1) $\mathrm{E}\left(\mathrm{B} 3 \mathrm{LYP} 6-31 \mathrm{G}^{*}\right)=-1658.69924$
$\mathrm{E}\left(\mathrm{B} 3 \mathrm{LYP} 6-311+\mathrm{G}^{* *}\right)=$
-1659.001017
$\mathrm{E}\left(\mathrm{B} 3 \mathrm{LYP} 6-31 \mathrm{G}^{*}\left(\mathrm{CH}_{3} \mathrm{CN}\right)\right)=$
-1658.743198
$\mathrm{E}\left(\mathrm{B} 3 \mathrm{LYP} 6-311+\mathrm{G}^{* *}\left(\mathrm{CH}_{3} \mathrm{CN}\right)\right)=$
-1659.04653

$\begin{array}{lccc}\text { C } & -1.58528 & -1.65585 & .31856 \\ \text { N } & -1.82081 & -.21752 & .46928 \\ \text { H } & -1.80980 & -2.04551 & -.68107 \\ \text { H } & .32711 & -1.00744 & -.49588 \\ \text { S } & -2.13478 & .59199 & -.91641 \\ \text { O } & -.90687 & 1.01581 & -1.64799 \\ \text { O } & -3.13305 & -.11654 & -1.76698 \\ \text { C } & -.11280 & -1.38356 & .42302 \\ \text { S } & 1.13212 & -3.36110 & .33631 \\ \text { C } & 2.77882 & -2.76964 & -.18863 \\ \text { H } & 2.69627 & -2.47504 & -1.23810 \\ \text { H } & 3.09671 & -1.91587 & .41472 \\ \text { H } & 3.49557 & -3.59112 & -.09769 \\ \text { C } & 1.45996 & -3.88374 & 2.05526 \\ \text { H } & 1.83592 & -3.05398 & 2.65733 \\ \text { H } & .51220 & -4.23678 & 2.46685 \\ \text { H } & 2.18376 & -4.70345 & 2.03637 \\ \text { C } & -2.90939 & 2.08256 & -.28233 \\ \text { H } & -3.11458 & 2.72386 & -1.14420 \\ \text { H } & -3.83784 & 1.81719 & .22701 \\ \text { H } & -2.22209 & 2.57881 & .40577 \\ \text { C } & -3.72055 & -4.15391 & 3.13808 \\ \text { C } & -3.76720 & -2.76223 & 3.26119 \\ \text { C } & -3.05767 & -1.95202 & 2.37185 \\ \text { C } & -2.30056 & -2.52099 & 1.34307 \\ \text { C } & -2.27278 & -3.91700 & 1.21154 \\ \text { C } & -2.96903 & -4.73063 & 2.10971 \\ \text { H } & -4.27547 & -4.78361 & 3.82969 \\ \text { H } & -4.36052 & -2.30336 & 4.04915 \\ \text { H } & -3.09882 & -.87157 & 2.45631 \\ \text { H } & -1.72785 & -4.37358 & .38716 \\ \text { H } & -2.94054 & -5.81151 & 1.99279 \\ \text { C } & .44545 & -.78589 & 1.68287 \\ \text { O } & 1.46054 & .04870 & 1.39503 \\ \text { O } & .08319 & -1.05696 & 2.80987 \\ \text { C } & 2.06895 & .72430 & 2.52233\end{array}$

\begin{abstract}
$\begin{array}{llll}\text { H } & 2.83732 & 1.36832 & 2.09422\end{array}$
H $1.32008 \quad 1.32089 \quad 3.04874$

H $2.51190 \quad .00074 \quad 3.21087$
\end{abstract}

\section{Syn elimination TS (conformer} 2)

$\mathrm{E}\left(\mathrm{B} 3 \mathrm{LYP} 6-31 \mathrm{G}^{*}\right)=-1658.707470$

$\mathrm{E}($ B3LYP 6-311+G**) $=$

$-1659.00085$

$\mathrm{E}\left(\mathrm{B} 3 \mathrm{LYP} 6-31 \mathrm{G}^{*}\left(\mathrm{CH}_{3} \mathrm{CN}\right)\right)=$ $-1658.742515$

$\mathrm{E}\left(\mathrm{B} 3 \mathrm{LYP} 6-311+\mathrm{G}^{* *}\left(\mathrm{CH}_{3} \mathrm{CN}\right)\right)=$ $-1659.045728$

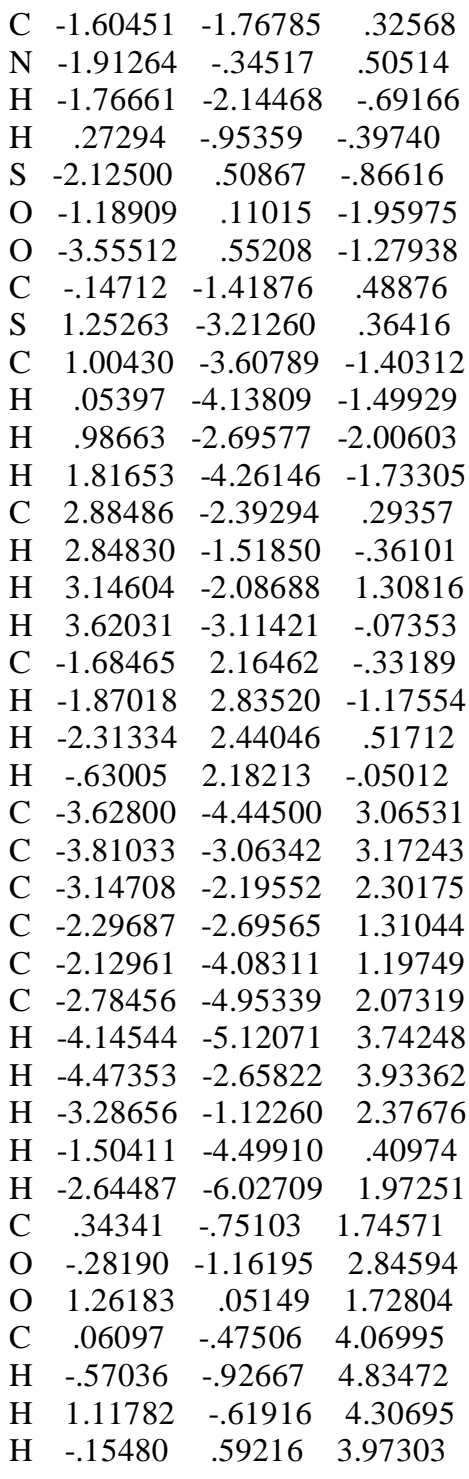

Syn elimination TS (conformer 3)

$\mathrm{E}\left(\mathrm{B} 3 \mathrm{LYP} 6-31 \mathrm{G}^{*}\right)=-1658.708856$ $\mathrm{E}\left(\mathrm{B} 3 \mathrm{LYP} 6-311+\mathrm{G}^{* *}\right)=$ $-1659.001989$ $\mathrm{E}\left(\mathrm{B} 3 \mathrm{LYP} 6-31 \mathrm{G}^{*}\left(\mathrm{CH}_{3} \mathrm{CN}\right)\right)=$
$-1658.742705$

$\mathrm{E}\left(\mathrm{B} 3 \mathrm{LYP} 6-311+\mathrm{G}^{* *}\left(\mathrm{CH}_{3} \mathrm{CN}\right)\right)=$ $-1659.046690$

$\begin{array}{lccc}\mathrm{C} & -1.61660 & -1.69688 & .32364 \\ \mathrm{~N} & -1.90986 & -.25989 & .36768 \\ \mathrm{H} & -1.77746 & -2.16682 & -.65449 \\ \mathrm{H} & .26725 & -1.00506 & -.49314 \\ \mathrm{~S} & -2.20915 & .42752 & -1.07919 \\ \mathrm{O} & -1.22684 & .03673 & -2.13396 \\ \mathrm{O} & -3.62961 & .26123 & -1.49667 \\ \mathrm{C} & -.15554 & -1.35246 & .44437 \\ \mathrm{~S} & 1.19581 & -3.20423 & .48375 \\ \mathrm{C} & 2.66409 & -2.54905 & -.38202 \\ \mathrm{H} & 2.40478 & -2.45933 & -1.44034 \\ \mathrm{H} & 2.93450 & -1.57122 & .02474 \\ \mathrm{H} & 3.48900 & -3.25826 & -.27123 \\ \mathrm{C} & 1.82623 & -3.36791 & 2.19057 \\ \mathrm{H} & 2.27113 & -2.42867 & 2.52879 \\ \mathrm{H} & .97941 & -3.63909 & 2.82464 \\ \mathrm{H} & 2.57303 & -4.16652 & 2.21213 \\ \mathrm{C} & -1.96446 & 2.16611 & -.70716 \\ \mathrm{H} & -2.18188 & 2.72482 & -1.62171 \\ \mathrm{H} & -2.65258 & 2.46046 & .08755 \\ \mathrm{H} & -.92909 & 2.32365 & -.39975 \\ \mathrm{C} & -3.67372 & -4.10885 & 3.27105 \\ \mathrm{C} & -3.80602 & -2.71724 & 3.29040 \\ \mathrm{C} & -3.12909 & -1.93168 & 2.35465 \\ \mathrm{C} & -2.31700 & -2.52686 & 1.38435 \\ \mathrm{C} & -2.20106 & -3.92426 & 1.35824 \\ \mathrm{C} & -2.86638 & -4.71164 & 2.30172 \\ \mathrm{H} & -4.20351 & -4.71926 & 3.99880 \\ \mathrm{H} & -4.44154 & -2.24043 & 4.03352 \\ \mathrm{H} & -3.23105 & -.85181 & 2.35946 \\ \mathrm{H} & -1.60540 & -4.40319 & .58392 \\ \mathrm{H} & -2.76746 & -5.79424 & 2.26734 \\ \mathrm{C} & .38808 & -.58396 & 1.61638 \\ \mathrm{O} & -.26380 & -.80728 & 2.75521 \\ \mathrm{O} & 1.37799 & .12363 & 1.51571 \\ \mathrm{C} & .13935 & -.00925 & 3.89190 \\ \mathrm{H} & -.50328 & -.33186 & 4.71045 \\ \mathrm{H} & 1.19047 & -.18498 & 4.13205 \\ \mathrm{H} & -.01859 & 1.05101 & 3.67735\end{array}$

\section{Anti elimination TS}

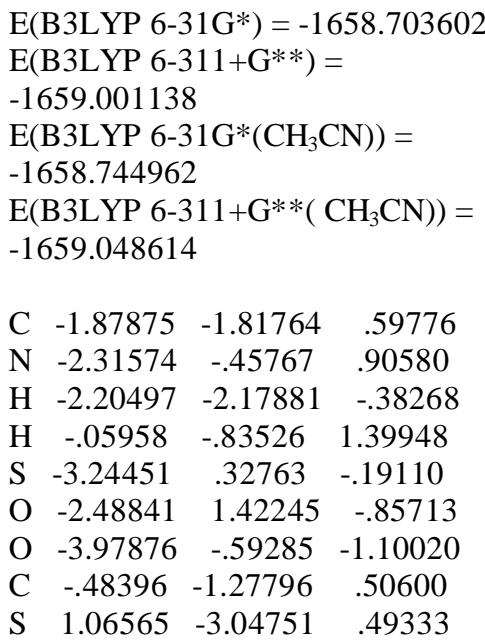




$\begin{array}{cccc}\mathrm{C} & 2.61610 & -2.34385 & -.17216 \\ \mathrm{H} & 2.50300 & -2.27403 & -1.25685 \\ \mathrm{H} & 2.80888 & -1.35631 & .25449 \\ \mathrm{H} & 3.43957 & -3.02573 & .05929 \\ \mathrm{C} & 1.44369 & -3.07570 & 2.28030 \\ \mathrm{H} & 1.69101 & -2.07281 & 2.64057 \\ \mathrm{H} & .55364 & -3.44685 & 2.79242 \\ \mathrm{H} & 2.27985 & -3.75763 & 2.45836 \\ \mathrm{C} & -4.46063 & 1.12694 & .87115 \\ \mathrm{H} & -5.10025 & 1.73978 & .22912 \\ \mathrm{H} & -5.04867 & .36005 & 1.37931 \\ \mathrm{H} & -3.93981 & 1.75503 & 1.59662 \\ \mathrm{C} & -2.85105 & -4.81716 & 3.55734 \\ \mathrm{C} & -2.86867 & -3.46323 & 3.90694 \\ \mathrm{C} & -2.54400 & -2.48804 & 2.96038 \\ \mathrm{C} & -2.19822 & -2.85654 & 1.65477 \\ \mathrm{C} & -2.18787 & -4.21573 & 1.30830 \\ \mathrm{C} & -2.50856 & -5.19234 & 2.25476 \\ \mathrm{H} & -3.11524 & -5.57465 & 4.29143 \\ \mathrm{H} & -3.14810 & -3.16379 & 4.91456 \\ \mathrm{H} & -2.58605 & -1.43455 & 3.21947 \\ \mathrm{H} & -1.94996 & -4.50930 & .28757 \\ \mathrm{H} & -2.50799 & -6.24190 & 1.97020 \\ \mathrm{C} & -.09826 & -.66996 & -.81650 \\ \mathrm{O} & .76050 & .34318 & -.67186 \\ \mathrm{O} & -.49293 & -1.09479 & -1.88534 \\ \mathrm{C} & 1.14040 & 1.02414 & -1.89509 \\ \mathrm{H} & 1.83515 & 1.80553 & -1.58766 \\ \mathrm{H} & 1.62091 & .32775 & -2.58623 \\ \mathrm{H} & .25179 & 1.45583 & -2.36071\end{array}$

\section{Cis aziridine}

$\mathrm{E}\left(\mathrm{B} 3 \mathrm{LYP} 6-31 \mathrm{G}^{*}\right)=-1180.697221$

$\mathrm{E}\left(\mathrm{B} 3 \mathrm{LYP} 6-311+\mathrm{G}^{* *}\right)=$

$-1180.986427$

$\mathrm{E}\left(\mathrm{B} 3 \mathrm{LYP} 6-31 \mathrm{G}^{*}\left(\mathrm{CH}_{3} \mathrm{CN}\right)\right)=$ $-1180.768284$

$\mathrm{E}\left(\mathrm{B} 3 \mathrm{LYP} 6-311+\mathrm{G} * *\left(\mathrm{CH}_{3} \mathrm{CN}\right)\right)=$ $-1181.016958$

$\begin{array}{cccc}\mathrm{C} & -1.24142 & -1.28214 & -.25360 \\ \mathrm{C} & .21440 & -.84619 & -.30808 \\ \mathrm{~N} & -.86205 & .12511 & -.07530 \\ \mathrm{H} & -1.44813 & -1.83322 & .66414 \\ \mathrm{H} & .81617 & -1.16821 & .53952 \\ \mathrm{~S} & -.96359 & .83472 & 1.48543 \\ \mathrm{O} & -1.13172 & -.19852 & 2.52419 \\ \mathrm{O} & .18008 & 1.75184 & 1.59505 \\ \mathrm{C} & -3.88883 & -2.40926 & -3.44729 \\ \mathrm{C} & -3.34174 & -1.12239 & -3.43179 \\ \mathrm{C} & -2.46461 & -.74106 & -2.41485 \\ \mathrm{C} & -2.12897 & -1.64698 & -1.39910 \\ \mathrm{C} & -2.68347 & -2.93564 & -1.41552 \\ \mathrm{C} & -3.55591 & -3.31671 & -2.43688 \\ \mathrm{H} & -4.57619 & -2.70113 & -4.23778 \\ \mathrm{H} & -3.60187 & -.40907 & -4.21041 \\ \mathrm{H} & -2.04338 & .25755 & -2.39931 \\ \mathrm{H} & -2.43803 & -3.63737 & -.62063 \\ \mathrm{H} & -3.98317 & -4.31648 & -2.43692 \\ \mathrm{C} & -2.48197 & 1.76923 & 1.32064 \\ \mathrm{H} & -2.63829 & 2.27353 & 2.27843 \\ \mathrm{H} & -2.35884 & 2.49474 & .51400\end{array}$

$\begin{array}{llll}\mathrm{H} & -3.30390 & 1.07978 & 1.11372\end{array}$

$\begin{array}{llll}\text { C } & .97581 & -.67856 & -1.59385\end{array}$

$\begin{array}{llll}\mathrm{O} & 2.14640 & -1.33230 & -1.52597\end{array}$

$\begin{array}{lllll}\mathrm{O} & .59260 & -.06273 & -2.56651\end{array}$

$\begin{array}{llll}\text { C } & 2.98252 & -1.26022 & -2.70571\end{array}$

$\begin{array}{llll}\mathrm{H} & 3.89353 & -1.80070 & -2.44845\end{array}$

$\begin{array}{llll}\mathrm{H} & 2.48117 & -1.73212 & -3.55454\end{array}$

H $\quad 3.20861 \quad-.21861 \quad-2.94585$

\section{Cis aziridine (conformer)}

$\mathrm{E}\left(\mathrm{B} 3 \mathrm{LYP} 6-31 \mathrm{G}^{*}\right)=-1180.709531$

$\mathrm{E}\left(\right.$ B3LYP $\left.6-311+\mathrm{G}^{* *}\right)=$

$-1180.985426$

$\mathrm{E}\left(\mathrm{B} 3 \mathrm{LYP} 6-31 \mathrm{G}^{*}\left(\mathrm{CH}_{3} \mathrm{CN}\right)\right)=$ $-1180.767773$

E(B3LYP 6-311+G** $\left.\left(\mathrm{CH}_{3} \mathrm{CN}\right)\right)=$ $-1181.016156$

$\begin{array}{lccccccc}\mathrm{C} & -1.33493 & -1.12367 & -.19869 & \mathrm{H} & -1.09157 & -.41603 & 2.56930 \\ \mathrm{C} & .16413 & -.88041 & -.23762 & \mathrm{H} & -2.35553 & -1.38010 & 4.47274 \\ \mathrm{~N} & -.78800 & .23938 & -.17927 & \mathrm{C} & .50126 & -1.17536 & -1.67302 \\ \mathrm{H} & -1.64252 & -1.53457 & .76371 & \mathrm{O} & 1.51196 & -2.05115 & -1.67256 \\ \mathrm{H} & -.68909 & -1.14947 & .67597 & \mathrm{O} & .03408 & -.65984 & -2.66939 \\ \mathrm{~S} & -.90928 & 1.12336 & 1.28519 & \mathrm{C} & 2.04540 & -2.41432 & -2.97095 \\ \mathrm{O} & -2.29013 & 1.62418 & 1.33932 & \mathrm{H} & 2.84840 & -3.12193 & -2.76478 \\ \mathrm{O} & -.41007 & .32864 & 2.42354 & \mathrm{H} & 1.26686 & -2.88165 & -3.57881 \\ \mathrm{C} & -3.86989 & -2.41136 & -3.42606 & \mathrm{H} & 2.43299 & -1.52981 & -3.48171\end{array}$

$\begin{array}{llll}\text { C } & -3.86989 & -2.41136 & -3.42606\end{array}$

$\begin{array}{llll}\text { C } & -3.28948 & -1.14035 & -3.47400\end{array}$

$\begin{array}{llll}\text { C } & -2.45643 & -.70611 & -2.44083\end{array}$

$\begin{array}{llll}\text { C } & -2.19936 & -1.54139 & -1.34585\end{array}$

$\begin{array}{llll}\text { C } & -2.79237 & -2.81217 & -1.29567\end{array}$

$\begin{array}{llll}\text { C } & -3.61932 & -3.24711 & -2.33329\end{array}$

H $\quad-4.51826 \quad-2.74675 \quad-4.23219$

H $\quad-3.48650 \quad-.48195 \quad-4.31683$

$\begin{array}{llll}\mathrm{H} & -2.00639 & .27950 & -2.47547\end{array}$

$\begin{array}{llll}\mathrm{H} & -2.61084 & -3.45910 & -.43951\end{array}$

$\begin{array}{llll}\mathrm{H} & -4.07345 & -4.23387 & -2.28474\end{array}$

$\begin{array}{llll}\text { C } & .21573 & 2.48015 & .96995\end{array}$

$\begin{array}{llll}\mathrm{H} & .18839 & 3.11188 & 1.86259\end{array}$

$\begin{array}{llll}\mathrm{H} & 1.22293 & 2.08540 & .81849\end{array}$

$\begin{array}{llll}\mathrm{H} & -.13341 & 3.03013 & .09372\end{array}$

$\begin{array}{llll}\text { C } & .97727 & -.96397 & -1.49818\end{array}$

$\begin{array}{llll}\text { O } & 2.06880 & -1.71642 & -1.29073\end{array}$

$\begin{array}{llll}\text { O } & .69685 & -.43329 & -2.55232\end{array}$

$\begin{array}{llll}\text { C } & 2.96396 & -1.86968 & -2.41936\end{array}$

H $3.78796 \quad-2.48283 \quad-2.05333$

$\begin{array}{llll}\mathrm{H} & 2.45161 & -2.36670 & -3.24626\end{array}$

$\begin{array}{llll}\mathrm{H} & 3.32568 & -.89154 & -2.74627\end{array}$

\section{Trans aziridine}

$\mathrm{E}\left(\mathrm{B} 3 \mathrm{LYP} 6-31 \mathrm{G}^{*}\right)=-1180.717234$

$\mathrm{E}\left(\mathrm{B} 3 \mathrm{LYP} 6-311+\mathrm{G}^{* *}\right)=$

$-1180.981470$

$\mathrm{E}\left(\mathrm{B} 3 \mathrm{LYP} 6-31 \mathrm{G}^{*}\left(\mathrm{CH}_{3} \mathrm{CN}\right)\right)=$ $-1180.766449$

$\mathrm{E}\left(\mathrm{B} 3 \mathrm{LYP} 6-311+\mathrm{G}^{* *}\left(\mathrm{CH}_{3} \mathrm{CN}\right)\right)=$ $-1181.013511$

$\begin{array}{llll}\text { C } & -1.51548 & -.86497 & -.09853\end{array}$

$\begin{array}{llll}\text { C } & -.00687 & -.96063 & -.27333\end{array}$
$\begin{array}{llll}\mathrm{N} & -.65236 & .28842 & .13678\end{array}$

$\begin{array}{lrrr}\mathrm{H} & .59396 & -1.41381 & .51100\end{array}$

$\begin{array}{llll}\text { S } & -.73714 & 1.73101 & -.78737\end{array}$

$\begin{array}{llll}\mathrm{O} & .63233 & 2.04650 & -1.21974\end{array}$

$\begin{array}{llll}\mathrm{O} & -1.82374 & 1.68177 & -1.77826\end{array}$

H $-2.05174 \quad-.86850 \quad-1.04747$

$\begin{array}{llll}\text { C }-1.22470 & 2.87532 & .50540\end{array}$

$\begin{array}{lll}\mathrm{H}-1.31557 & 3.85726 & .03128\end{array}$

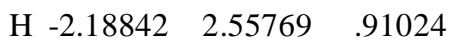

$\begin{array}{llll}\mathrm{H} & -.45436 & 2.88492 & 1.27844\end{array}$

$\begin{array}{llll}\text { C } & -3.69315 & -2.52038 & 3.21649\end{array}$

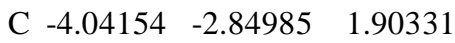

$\begin{array}{llll}\text { C } & -3.32755 & -2.30737 & .83191\end{array}$

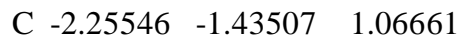

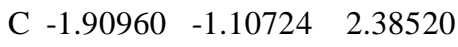

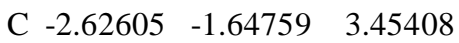

H $-4.25363 \quad-2.93571 \quad 4.05052$

H $-4.87397 \quad-3.52249 \quad 1.71170$

H $-3.60728 \quad-2.55612 \quad-.18983$

H $-1.09157 \quad-.41603 \quad 2.56930$

H $-2.35553 \quad-1.38010 \quad 4.47274$

$\begin{array}{llll}\text { C } & .50126 & -1.17536 & -1.67302\end{array}$

$\begin{array}{llll}\text { O } & 1.51196 & -2.05115 & -1.67256\end{array}$

$\begin{array}{llll}\text { C } & 2.04540 & -2.41432 & -2.97095\end{array}$

$\begin{array}{llll}\mathrm{H} & 2.84840 & -3.12193 & -2.76478\end{array}$

H $2.43299 \quad-1.52981 \quad-3.48171$

Trans aziridine (conformer 1)

$\mathrm{E}\left(\mathrm{B} 3 \mathrm{LYP} 6-31 \mathrm{G}^{*}\right)=-1180.703850$

$\mathrm{E}\left(\mathrm{B} 3 \mathrm{LYP} 6-311+\mathrm{G}^{* *}\right)=$

$-1180.980418$

$\mathrm{E}\left(\mathrm{B} 3 \mathrm{LYP} 6-31 \mathrm{G} *\left(\mathrm{CH}_{3} \mathrm{CN}\right)\right)=$

$-1180.763773$

$\mathrm{E}\left(\mathrm{B} 3 \mathrm{LYP} 6-311+\mathrm{G}^{* *}\left(\mathrm{CH}_{3} \mathrm{CN}\right)\right)=$ $-1181.012013$

$\begin{array}{cccc}\mathrm{C} & -1.55145 & -.84554 & .13802 \\ \mathrm{C} & -.07450 & -.97776 & -.06087 \\ \mathrm{~N} & -.67012 & .36063 & .14490 \\ \mathrm{H} & .51697 & -1.38612 & .75399 \\ \mathrm{~S} & -.32065 & 1.21230 & 1.59331 \\ \mathrm{O} & -1.57379 & 1.87397 & 1.98298 \\ \mathrm{O} & .37517 & .36748 & 2.58095 \\ \mathrm{H} & -2.14252 & -.82900 & -.77662 \\ \mathrm{C} & .82960 & 2.44264 & .97373 \\ \mathrm{H} & 1.09436 & 3.07044 & 1.83019 \\ \mathrm{H} & 1.71822 & 1.94133 & .58322 \\ \mathrm{H} & .33229 & 3.03115 & .20041 \\ \mathrm{C} & -3.83799 & -2.42686 & 3.42400 \\ \mathrm{C} & -4.37211 & -1.44614 & 2.58152 \\ \mathrm{C} & -3.60607 & -.93191 & 1.53575 \\ \mathrm{C} & -2.29330 & -1.38460 & 1.32458 \\ \mathrm{C} & -1.76460 & -2.36762 & 2.17045 \\ \mathrm{C} & -2.53497 & -2.88585 & 3.21537 \\ \mathrm{H} & -4.43762 & -2.83544 & 4.23394 \\ \mathrm{H} & -5.38702 & -1.08715 & 2.73442 \\ \mathrm{H} & -4.02852 & -.17877 & .87435 \\ \mathrm{H} & -.75996 & -2.74796 & 2.00960 \\ \mathrm{H} & -2.11727 & -3.65488 & 3.86043 \\ \mathrm{C} & .45255 & -1.19964 & -1.45339\end{array}$


O $\quad \begin{array}{llll}1.54793 & -1.96462 & -1.44858\end{array}$

$\begin{array}{lllll}\mathrm{O} & -.06741 & -.74485 & -2.45340\end{array}$

C $\quad 2.12133 \quad-2.25799 \quad-2.74785$

H $2.99096 \quad-2.88289-2.54629$

H $\quad 1.39538 \quad-2.79153 \quad-3.36586$

H $\quad 2.41755 \quad-1.33082 \quad-3.24461$

\section{Trans aziridine (conformer 2)}

$\mathrm{E}\left(\mathrm{B} 3 \mathrm{LYP} 6-31 \mathrm{G}^{*}\right)=-1180.696867$

$\mathrm{E}\left(\mathrm{B} 3 \mathrm{LYP} 6-311+\mathrm{G}^{* *}\right)=$

$-1180.977554$

$\mathrm{E}\left(\mathrm{B} 3 \mathrm{LYP} 6-31 \mathrm{G}^{*}\left(\mathrm{CH}_{3} \mathrm{CN}\right)\right)=$

$-1180.762409$

$\mathrm{E}\left(\right.$ B3LYP $\left.6-311+\mathrm{G}^{* *}\left(\mathrm{CH}_{3} \mathrm{CN}\right)\right)=$ $-1181.010811$

$\begin{array}{lccc}\mathrm{C} & -1.58797 & -.93080 & .14473 \\ \mathrm{C} & -.10465 & -.98630 & -.03104 \\ \mathrm{~N} & -.77399 & .32241 & .15346 \\ \mathrm{H} & .49474 & -1.36406 & .79310 \\ \mathrm{~S} & -.37158 & 1.21260 & 1.56220 \\ \mathrm{O} & -.31854 & .37470 & 2.77159 \\ \mathrm{O} & .83751 & 1.96663 & 1.19521 \\ \mathrm{H} & -2.16157 & -.94934 & -.78176 \\ \mathrm{C} & -1.76330 & 2.33630 & 1.66204 \\ \mathrm{H} & -1.53028 & 3.03821 & 2.46825 \\ \mathrm{H} & -1.85956 & 2.85990 & .70845 \\ \mathrm{H} & -2.66552 & 1.77015 & 1.90163 \\ \mathrm{C} & -3.81213 & -2.50064 & 3.47339 \\ \mathrm{C} & -4.33787 & -1.47518 & 2.68114 \\ \mathrm{C} & -3.59933 & -.97523 & 1.60786 \\ \mathrm{C} & -2.31923 & -1.48087 & 1.32989 \\ \mathrm{C} & -1.80321 & -2.51477 & 2.11886 \\ \mathrm{C} & -2.54855 & -3.02331 & 3.18512 \\ \mathrm{H} & -4.38872 & -2.89546 & 4.30626 \\ \mathrm{H} & -5.32414 & -1.06963 & 2.89299 \\ \mathrm{H} & -4.02275 & -.19633 & .97608 \\ \mathrm{H} & -.82457 & -2.93222 & 1.89895 \\ \mathrm{H} & -2.14069 & -3.82988 & 3.78910 \\ \mathrm{C} & .45833 & -1.17731 & -1.41706 \\ \mathrm{O} & 1.62841 & -1.82044 & -1.39858 \\ \mathrm{O} & -.09890 & -.79378 & -2.42643 \\ \mathrm{C} & 2.24213 & -2.04530 & -2.69468 \\ \mathrm{H} & 3.17015 & -2.57801 & -2.48898 \\ \mathrm{H} & 1.58201 & -2.64630 & -3.32413 \\ \mathrm{H} & 2.44604 & -1.08868 & -3.18197\end{array}$

\section{D. $\mathrm{CH}_{2} \mathrm{SMe}_{2}+$ $\mathrm{PhCH}=\mathrm{NSO}_{2} \mathrm{Me}$}

\section{Cisoid addition TS}

$\mathrm{E}\left(\mathrm{B} 3 \mathrm{LYP} 6-31 \mathrm{G}^{*}\right)=-1430.819909$

$\mathrm{E}($ B3LYP 6-311+G**) $=$

$-1431.056463$

$\mathrm{E}\left(\mathrm{B} 3 \mathrm{LYP} 6-31 \mathrm{G}^{*}\left(\mathrm{CH}_{3} \mathrm{CN}\right)\right)=$

$-1430.848626$

$\mathrm{E}\left(\mathrm{B} 3 \mathrm{LYP} 6-311+\mathrm{G}^{* *}\left(\mathrm{CH}_{3} \mathrm{CN}\right)\right)=$

$-1431.086401$

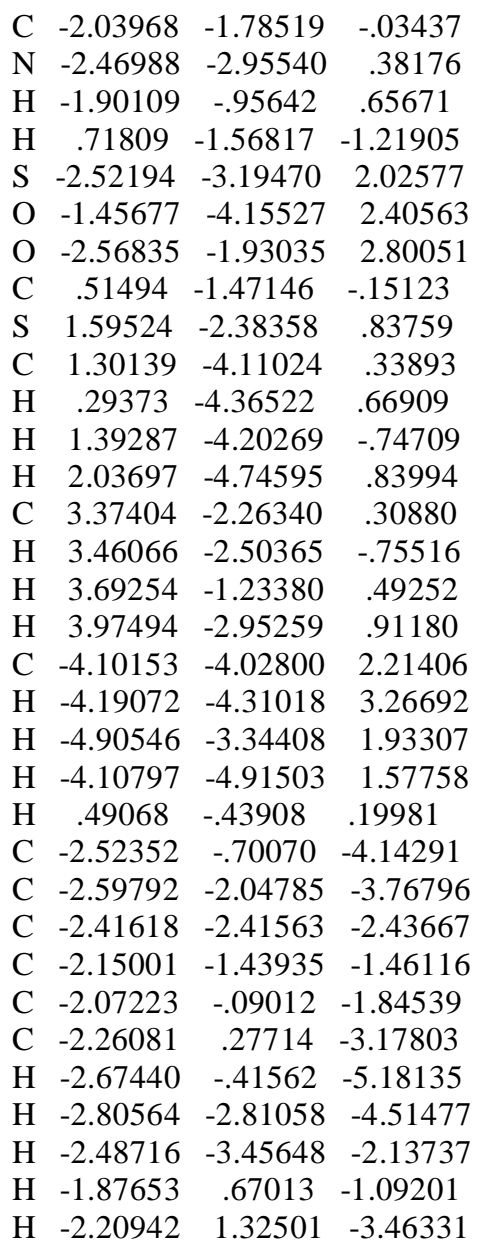

Cisoid addition TS (conformer)

$\mathrm{E}\left(\mathrm{B} 3 \mathrm{LYP} 6-31 \mathrm{G}^{*}\right)=-1430.820344$

$\mathrm{E}\left(\mathrm{B} 3 \mathrm{LYP} 6-311+\mathrm{G}^{* *}\right)=$

$-1431.057517$

$\mathrm{E}\left(\mathrm{B} 3 \mathrm{LYP} 6-31 \mathrm{G}^{*}\left(\mathrm{CH}_{3} \mathrm{CN}\right)\right)=$ $-1430.848119$

$\mathrm{E}\left(\mathrm{B} 3 \mathrm{LYP} 6-311+\mathrm{G} * *\left(\mathrm{CH}_{3} \mathrm{CN}\right)\right)=$ $-1431.0857977$

$\begin{array}{lcll}\mathrm{C} & -2.11106 & -1.78792 & .20356 \\ \mathrm{~N} & -2.54073 & -2.87342 & .78967 \\ \mathrm{H} & -1.97595 & -.85840 & .75418 \\ \mathrm{H} & .58261 & -.56509 & -.23734 \\ \mathrm{~S} & -2.69331 & -2.82979 & 2.45387 \\ \mathrm{O} & -1.72471 & -3.78015 & 3.04266 \\ \mathrm{O} & -2.69666 & -1.45439 & 3.00606 \\ \mathrm{C} & .55642 & -1.38483 & .47810 \\ \mathrm{~S} & 1.55420 & -2.66394 & -.05718 \\ \mathrm{C} & 3.32656 & -2.56652 & .50953 \\ \mathrm{H} & 3.77681 & -1.71156 & -.00201 \\ \mathrm{H} & 3.36491 & -2.42277 & 1.59374 \\ \mathrm{H} & 3.84575 & -3.48833 & .22560 \\ \mathrm{C} & 1.06534 & -4.09212 & .96313 \\ \mathrm{H} & 1.18267 & -3.85167 & 2.02293 \\ \mathrm{H} & .01648 & -4.29664 & .74832 \\ \mathrm{H} & 1.69184 & -4.94630 & .69091 \\ \mathrm{C} & -4.33318 & -3.52649 & 2.66669 \\ \mathrm{H} & -4.50660 & -3.61918 & 3.74281\end{array}$

$\begin{array}{llll}\mathrm{H} & -5.06673 & -2.85566 & 2.21441\end{array}$

$\begin{array}{llll}\mathrm{H} & -4.35677 & -4.50788 & 2.18922\end{array}$

$\begin{array}{llll}\mathrm{H} & .69791 & -1.09107 & 1.51961\end{array}$

C $\quad-2.32793 \quad-1.43267 \quad-4.04809$

$\begin{array}{llll}\text { C } & -2.43466 & -2.69554 & -3.45215\end{array}$

$\begin{array}{llll}\text { C } & -2.33428 & -2.82814 & -2.06890\end{array}$

$\begin{array}{llll}\text { C } & -2.11636 & -1.69669 & -1.26356\end{array}$

$\begin{array}{llll}\text { C } & -2.00321 & -.43360 & -1.86914\end{array}$

$\begin{array}{llll}\text { C } & -2.11254 & -.30191 & -3.25326\end{array}$

H $\quad-2.41740 \quad-1.33085-5.12701$

$\mathrm{H} \quad-2.60604 \quad-3.57546-4.06775$

$\begin{array}{llll}\mathrm{H} & -2.43572 & -3.80149 & -1.59854\end{array}$

$\begin{array}{llll}\mathrm{H} & -1.84472 & .44513 & -1.24764\end{array}$

$\begin{array}{llll}\text { H } & -2.03669 & .68098 & -3.71160\end{array}$

\section{transoid addition TS}

$\mathrm{E}\left(\mathrm{B} 3 \mathrm{LYP} 6-31 \mathrm{G}^{*}\right)=-1430.816949$

$\mathrm{E}\left(\mathrm{B} 3 \mathrm{LYP} 6-311+\mathrm{G}^{* *}\right)=$

$-1431.056969$

$\mathrm{E}\left(\mathrm{B} 3 \mathrm{LYP} 6-31 \mathrm{G} *\left(\mathrm{CH}_{3} \mathrm{CN}\right)\right)=$

$-1430.848036$

$\mathrm{E}\left(\mathrm{B} 3 \mathrm{LYP} 6-311+\mathrm{G}^{* *}\left(\mathrm{CH}_{3} \mathrm{CN}\right)\right)=$ $-1431.085159$

$$
\begin{array}{cccc}
\mathrm{C} & -1.88086 & -1.615348 & .45559 \\
\mathrm{~N} & -2.03645 & -.316355 & .32029 \\
\mathrm{H} & -1.87328 & -2.281038 & -.40458 \\
\mathrm{H} & .92434 & -1.081608 & .87291 \\
\mathrm{~S} & -1.93487 & .313508 & -1.21125 \\
\mathrm{O} & -.68919 & 1.106750 & -1.33537 \\
\mathrm{O} & -2.17551 & -.696138 & -2.27456 \\
\mathrm{C} & .58967 & -2.096776 & .64998 \\
\mathrm{~S} & 1.49999 & -2.872763 & -.59772 \\
\mathrm{C} & 1.24589 & -1.833030 & -2.07199 \\
\mathrm{H} & .19062 & -1.892218 & -2.34192 \\
\mathrm{H} & 1.50820 & -.796618 & -1.84473 \\
\mathrm{H} & 1.87088 & -2.222876 & -2.88031 \\
\mathrm{C} & 3.34162 & -2.651371 & -.45186 \\
\mathrm{H} & 3.58680 & -1.587496 & -.37925 \\
\mathrm{H} & 3.65317 & -3.175852 & .45533 \\
\mathrm{H} & 3.82392 & -3.102287 & -1.32592 \\
\mathrm{C} & -3.31202 & 1.466583 & -1.20582 \\
\mathrm{H} & -3.28227 & 2.007578 & -2.15597 \\
\mathrm{H} & -4.24627 & .908975 & -1.11130 \\
\mathrm{H} & -3.18562 & 2.155470 & -.36788 \\
\mathrm{H} & .56072 & -2.753454 & 1.51859 \\
\mathrm{C} & -2.88964 & -3.441381 & 4.19248 \\
\mathrm{C} & -2.66365 & -2.061214 & 4.13361 \\
\mathrm{C} & -2.30994 & -1.459326 & 2.92702 \\
\mathrm{C} & -2.17000 & -2.233647 & 1.76277 \\
\mathrm{C} & -2.39064 & -3.619163 & 1.83161 \\
\mathrm{C} & -2.75287 & -4.219153 & 3.03727 \\
\mathrm{H} & -3.17426 & -3.907419 & 5.13291 \\
\mathrm{H} & -2.77089 & -1.452710 & 5.02857 \\
\mathrm{H} & -2.15152 & -.386722 & 2.87249 \\
\mathrm{H} & -2.28521 & -4.221145 & .93145 \\
\mathrm{H} & -2.93358 & -5.290580 & 3.07659
\end{array}
$$

